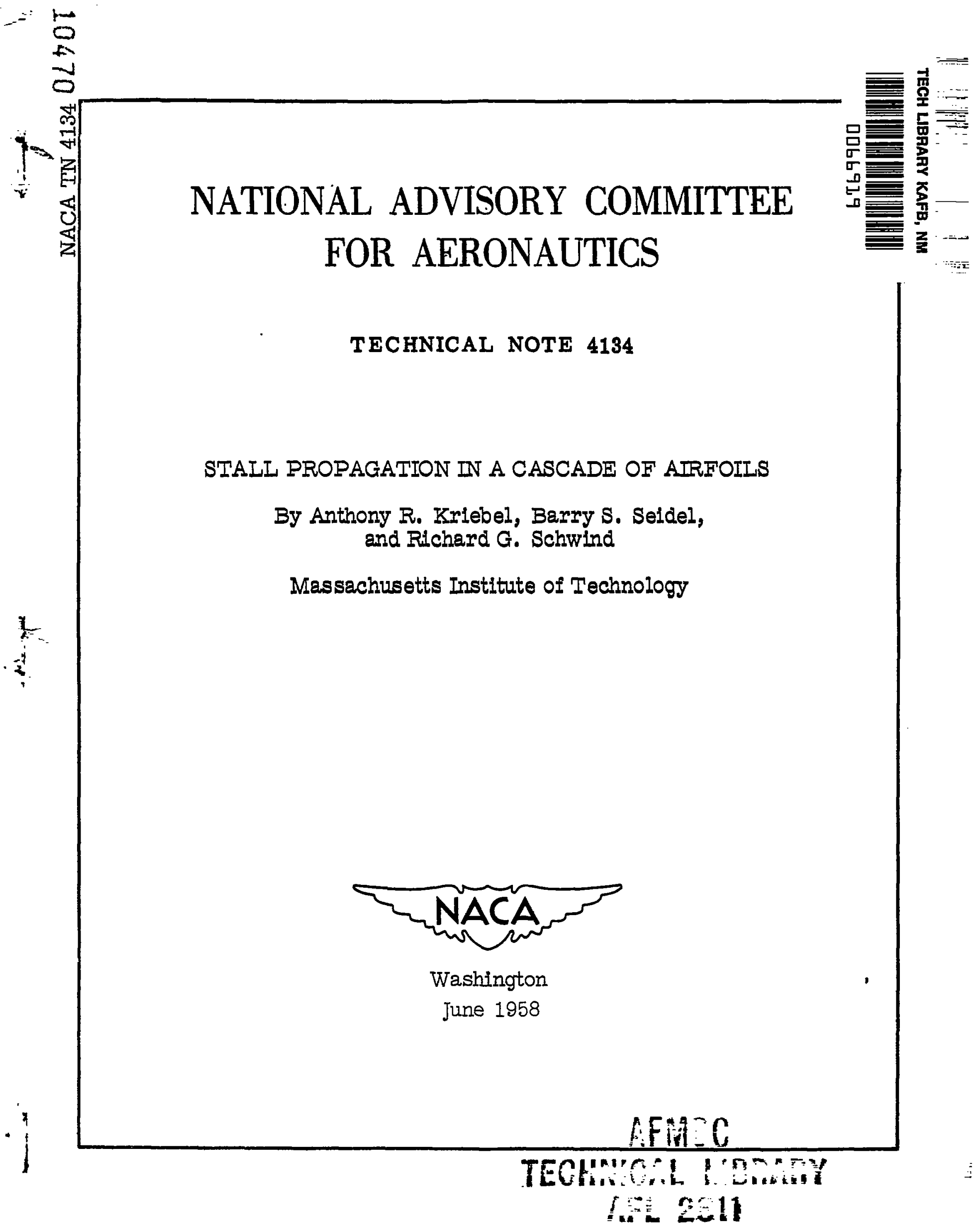


TECHNICAL NOTE 4134

\section{STALL PROPAGATION IN A CASCADE OF AIRFOIIS}

By Anthony R. Kriebel, Barry S. Seidel, and Richard G. Schwind

\section{SUMMARY}

An experimental investigation of stall propagation in a stationary circular cascade in which high-speed schlieren and interferometer photography is used is described. This investigation suggests an analytical approach to the study of stall propagation which is valid only for an isolated blade row in an infinite flow field but which is not restricted to small unsteady perturbations or to an assumed simplified cascade geometry. Conditions necessary for the existence of the assumed type of stall cells are described and equations are derived for the velocity of stall-cell propagation.

The propagation velocities predicted for the theoretical potentialflow model correlate with all the experimental values measured in an isolated rotor within 15 percent.

Analysis of the flow model leads to the prediction of a tendency for the assumed type of stall cell to split with increasing incldence of the mean flow through the blade row. This tendency appears to correlate with the experimental observation of a trend for increasing numbers of cells in the rotor.

\section{INTRODUCTION}

The objective of the analytical and experimental work presented herein is the development of a theory which will enable the prediction of the flow through a cascade of rigid airfoils, or an isolated blade row of an axial compressor, when the incidence of the fluid on the airfoils is high. It was discovered in the early days of British jetengine development that the flow can be unstable under these conditions and that self-induced periodic disturbances on the flow can develop. The disturbances are caused by the propagation along the cascade, at approximately the relative tangential component of main-stream velocity, of regions where the flow is badly separated from the airfoils. These regions where the blades are severely stalled are generally called stall cells. Stall-cell propagation in axial compressors has continued to 
recelve considerable attention since the pioneering analytical treatment by Fmoñs (ref. l) because of its importance as a cause of blade fatigue failure.

The appearance of stall propagation in aircraft gas turbine engines generally occurs in the early stages of the axial compressor during any off-design operating condition in which these stages operate at inc1dences much higher than design. This condition can arise because of restrlction of flow assoclated with engine acceleration or because of operation at lower than the design value of reduced engine speed $N / \sqrt{T}$. Several mechanical devices are in use which tend to prevent the occurrence of rotating stall by lowering the incidence in the first few stages during such off-design operation. However, there are weight penalties and/or aerodynamic losses associated with these devices.

A satisfactory stall propagation theory might lead to (I) prevention of unsteady flow due to stall propagation when the incidence of the mean flow is high, (2) alteration of the stall-cell pattern and/or its velocity of propagation so that, for a given cascade geometry and mean flow, the forcing frequency of the blade loading can be chosen by the designer, or (3) prediction of enough information about the unsteady flow so that blades can be designed to withstand the unsteady aerodynamic loading.

However, before such a theory can be achieved, it appears that more facts must be known about the detalled nature of the flow during stall propagation in a blade row than have appeared in the literature. Experiments were run in a radial-outflow circular cascade installed in a closed-circult wind tunnel in order to take high-speed schlieren and interferometer pictures of the flow through a portion of the cascade during stall propagation. Further experiments were run with an isolated rotor of an axial flow compressor. The data taken in these two test rigs suggest certain approximations to the flow which ald in the subsequent analytical treatment of the problem.

The analysis of the general problem of stall propagation in axial compressors is obviously difficult since an unsteady, rotational, threedimensional flow of a compressible fluid is involved. In order to retain the essential features of the flow but to simplify the problem as much as possible for analytical treatment, the flow is assumed to be twodimensional and incompressible and to be limited to the case of an isolated blade row. It is belleved that further analysis of this problem is necessary before the much more difficult three-dimensional and mult1stage flow problem can be successfuily attacked.

There have been several analyses of the problem with the restricting assumptions given above. (These analyses are given in refs. 1, 2, 3, and 4. A brief summary of each is presented in ref. 4.) The main reason for attempting another analysis of the same problem is the fact that all 
of the known previous theoretical work has been further restricted by the assumption that the unsteady velocity perturbations are small with respect to the mean flow velocity. From the experimental data presented herein and in the references, this assumption does not appear to be a good approximation; thus the present analysis is not restricted to small unsteady perturbations. Moreover, the previous analyses have been based upon rather restrictive representations of an isolated blade row, either as a lifting line or as infinitesimally spaced flat plates; whereas, in the present analysis, the chord, spacing, shape, and detailed stall characteristics of the blades are not specified. Finally, none of the theories, except that given in reference 4, have offered apparent physical causes which determine the number of stall cells. The analytical flow model used herein appears to present qualitative information in this regard.

From information available at present, it appears that the desirable features of a stall propagation theory are predictions for a given cascade geometry of:

(1) mean flow incidences at which stall propagation is possible

(2) the number of stall cells

(3) the velocity of stali-cell propagation

(4) the magnitude of unsteady aerodynamic forces on the airfoils

Such a theory is the goal toward which the work presented herein is directed.

This report summarizes the results of an investigation in which many members of the M.I.T. Gas Turbine Laboratory staff have participated.

Profs. E. S. Taylor and A. H. Stenning guided the project. Prof. H. E. Edgerton generously lent one of his experimental light sources and advised in its use during the project. Prof. E. I. Mollo-Christensen provided the miniature piezoelectric crystal pressure pickup and advised in 1ts use during the project.

This investigation was conducted at the Massachusetts Institute of Technology under the sponsorship and with the financial assistance of the National Advisory Committee for Aeronautics. 


\section{SYMBOLS}

A

discharge coefficient, $\cos \beta_{1} / \cos \beta_{2} \sqrt{1-C_{p}}$

a vortex spacing along stall cell downstream of blade row

B number of stall cells

C absolute velocity

$\mathrm{C}_{\mathrm{p}}$ static pressure coefficlent of cascade, $\frac{p_{2}-p_{1}}{\frac{1}{2} \mathrm{pW}_{1}{ }^{2}}$

$\mathrm{C}_{\mathrm{I}}$ blade lift coefficlent, $\frac{\text { Lift/Area }}{\frac{1}{2} \mathrm{pW}_{1}^{2}}$

blade normal-force coefficient, Aerodynamic force normal to chord

$$
\frac{1}{2} \rho W_{1}^{2}
$$

$F$

frequency at which each airfoil stalls

$f()$

function of

1 Incidence, $\beta-\lambda, \sqrt{-1}$

K defined by equation (7)

L blade chord

$\imath \quad$ length of cascade or circumference of rotor

M Mach number

m length of stall cell in vortex spaces a

$\mathrm{N}=\frac{\mathrm{V}_{1} \cos \phi}{\mathrm{W}_{\mathrm{x}_{2}}}$

$n$ width of stall cell downstream of blade row in vortex spaces a $P=\Gamma / \Gamma_{0}$ 
4

$$
\begin{aligned}
& \mathrm{p} \text { static pressure } \\
& \mathrm{R} \quad \text { radius of cylinder in picture plane, } \frac{\mathrm{L}}{4} \\
& \mathrm{Re} \quad \text { Reynolds number based on blade chord, } \frac{\rho \mathrm{WL}}{\mu} \\
& \mathrm{S}=\frac{\text { Time } \frac{\operatorname{mean} \mathrm{p}_{0}-\mathrm{p}}{\frac{1}{2} \mathrm{\rho W}_{1}{ }^{2}}}{}
\end{aligned}
$$

s cascade blade spacing

t time

U rotor velocity at mean radius, $W_{\theta_{1}}$ for isolated rotor

$u$, v velocity components in $x, y$ directions

$V \quad$ transport velocity of vortex downstream of blade row

$v_{a} \quad$ velocity of vortex $\Gamma$ along lines (b) and (c) in figure io

$\mathrm{V}_{1} \quad$ velocity induced by stall cell, $\mathrm{T} / \mathrm{a}$

$V_{p} \quad$ velocity of stall propagation relative to blade row

$V_{Q} \quad$ absolute velocity of stall propagation, $U-V_{P}$

W velocity relative to cascade or rotor

$\mathrm{X}=\frac{\mathrm{W}_{\mathrm{x}_{1}}}{\mathrm{~W}_{\mathrm{x}_{2}}}$

$Y$

fraction of downstream periphery covered by stall cells, $\frac{n a}{2 \cos \phi}$

z complex coordinate in physical plane, $x+i y$

$\alpha \quad$ angle of flow from axial direction in absolute coordinate system 
B angle of flow from axial direction in coordinate system fixed to blade row

$\Gamma \quad$ strength of shed vortex

$\Gamma_{0} \quad$ strength of bound vortex

$\zeta \quad$ complex coordinate in picture plane, $\xi+1 \eta$

$\theta$ polar coordinate; blade camber angle

$\lambda \quad$ cascade stagger angle from blade chord to axial direction

$\rho \quad$ density of fluid

$\sigma \quad$ cascade solidity, L/s

T period with whtch stall cells pass a fixed point

$\phi \quad$ angle of stall cell from axial direction

$\psi \quad$ velocity potential

(7) complex conjugate

$\left({ }^{0}\right)=\partial() / \partial t$

Subscripts :

$1 \quad$ Inside stall cell

- stagnation point; outside stall cell

1 far upstream; upstream measuring station in circular cascade

2 far downstream; downstream measuring station in circular cascade

C, E, F, FG, G, $K$ measuring stations along axts of rotor shown in figure 35

$r \quad$ radial component

$\mathrm{x} \quad$ axial component (note, $\mathrm{c}_{\mathbf{x}}=\mathrm{W}_{\mathbf{x}}$ )

$\theta \quad$ tangential component 
Conditions required for the existence of small-perturbation stall propagation and a mechanism by which the stalling of rigid airfoils in cascade can propagate have been clearly explained in references $I$ and 4, where the cascade is assumed to consist of infinitesimally spaced flat plates of finite chord which stall at or near their tralling edges and between which the flow is one-dimensional. It is apparent that, as is described in these references, the diversion of flow around a stalled airfoil tends to stall the adjacent airfoil on its suction side and to unstaII the opposite adjacent airfoil. However, in order to apply the analysis of reference 4 to calculate the velocity of stall propagation, it is necessary to know an "equivalent chord length" if the airfoils do not stall locally at their trailing edges. In general, from the data presented herein, it appears that the separation of the boundary layer associated with stalling occurs more nearly at the airfoil leading edge during stall propagation and that the flow in the blade passages is rather far from one-dimensional for typical values of cascade solidity $\sigma$. It was further assumed in reference 4 that the performance of a blade, that is, the "discharge coefficient A," or blade boundary-layer displacement thickness, was a function only of local incldence. A result of the analysis based upon these assumptions was that the harmonics of the small perturbation traveled at different velocities and it became necessary to restrict the velocity perturbation upstream of the cascade to a pure sine wave in the analysis in order that it should maintain its shape as it propagated. The sine-wave shape does not agree well with experimental data, particularly for small numbers of cells.

In all the previous analyses, the equations of motion were solved for the irrotational motion of the fluid upstream of the cascade with the cascade as a boundary and the cascade characteristics appearing in the boundary conditions. Since the time-depepdent differential equations are nonlinear, the assumption of small unsteady perturbations was necessary in order to linearize and solve the equations. Furthermore, in order to make the boundary conditions continuous, it was necessary to restrict the cascade geometry to infinitesimal blade spacing. The analyses based upon the assumption of small perturbations have yielded valuable information about the qualitative nature of stali propagation. Undoubtedly this assumption is valid for the prediction of the onset of propagation; however, its validity for the prediction of propagation velocities of large amplitude perturbations appears uncertain.

In reference 3 an analysis based upon representation of the cascade as a distributed vortex sheet (infinitesimal blade chord and spacing) led to the prediction of infinite velocity of propagation for some values of incidence unless an arbitrary "boundary layer phase lag" was included. This difficulty did not arise in the analyses of references 2 and 4, 
where the assumption of infinitesimal blade spacing was made also. From the analysis herein, where the two-dimensional flow between finitely spaced airfoils is considered in representing the cascade during stall propagation, it appears that the propagation velocity must be of the same order of magnitude as the free-stream velocity even if the boundarylayer phase lag of the airfoils is zero. As the dimensions of the cascade shrink to zero, similitude requires that $V_{p}$ remain finite. The present analysis indicates also that the stall cells must be regions of high losses and low stagnation pressure in a coordinate system fixed to the stall-cell pattern, which is contrary to the assumption of constant stagnation pressure in the section "Airfoll Theory" of reference 3.

Information derived from high-speed interferometer plctures of the flow through a portion of a cascade during stall propagation suggests an analytical approach which does not necessitate the direct integration of the equations of motion, which is not limited to small perturbations, infinitesimal blade chord, or infinltesimal blade spacing, and which does not require arbitrary assumptions regarding the location of separation of the boundary layer from the airfoil or the time required for separation.

\section{ANALYSIS OF STALI PROPAGATION IN A BLADE ROW}

\section{Development of a Vortex Flow Model}

Experimental and mathematical justification. - It is noted in the interferometer pictures of rotating stall in circular cascade (figs. I to 4) that the vorticity shed downstream from the cascade airfoils appears to be concentrated largely in discrete vortices which accumulate at and depart from the leading and trailing edges of the airfoils as they periodically stall and unstall. Furthermore, the pictures indicate that the airfoils shed a large part of their bound vorticity when they stall out. This appears to be true for all the circular cascade conflgurations, Reynolds numbers, and Mach numbers tested. Experimental data and calculations which support these conclusions and which suggest that they may be valid also for the flow through a research compressor assembled as an isolated rotor are presented subsequently. In general, the data suggest that the airfoils which were tested, both in the rotor and in cascade, shed vortices during stall propagation similar to the Kármán vortex street shed from a flat plate and that the vortices formed in a manner similar to the vortices formed at the edge of a flat plate moved impulsively from rest normal to surface of the plate, as in the analysis of reference 5 .

However, the first suggestion that there might be a strong connection between stall propagation and vortex shëding was in reference 4 , 
Where it was observed experimentally that, 'as the solidity of the circuIar cascade was reduced, the frequency of stall propagation approached the Kármán vortex-street frequency for an isolated plate as found experimentally in reference 6. Similar data for a different configuration of the circular cascade are shown in figure 5.

With the above experimental evidence as a basis, it is assumed that during stall propagation all the circulation downstream of the cascade is about discrete potential vortices which are shed from the airfoils when they alter their circulation upon entering or leaving a stall cell. The experimental evidence suggests that with this assumption, it should be possible to devise a vortex flow model of stall propagation in a blade row where the vortex shed from the leading edge of a blade moves across the passage and stalls the adjacent blade before moving on downstream with undiminished strength.

There are several advantages which arise from the fact that the hypothetical flow model is irrotational except at discrete singularities. The most important advantage is that the flow, even though it is unsteady, is "kinematic;" that is, the equations of motion need not be integrated to solve the flow, since the assumed conditions of irrotationality, continuity, and incompressibility alone are sufficient. This may be shown from the vector identity

$$
\nabla^{2} \mathrm{~V}=\operatorname{grad} \mathrm{div} \mathrm{V}-\operatorname{curl} \operatorname{curl} \mathrm{V}
$$

The continuity equation,

$$
\frac{\partial \rho}{\partial t}+d i v \rho V=0
$$

shows that div V $=0$ since $\rho$ is assumed constant. Stokes' and Kelvin's theorems are not affected by time varlation of flow and are sufficient to determine irrotationality. Since all vorticity is assumed to be concentrated at singular points and inside airfoil surfaces, curl $V=0$ everywhere in the flow field because the singular points are excluded from the field. Consequently, $\nabla^{2} \mathrm{~V}=0$. Laplace's equation is satisfied at every instant of time, and the flow may be built up by the superposition of the unsteady potential flows about the vortices which are assumed to be shed from the airfoils and convected downstream.

If the effect of a single potential vortex upon the flow about an isolated flat plate is studied, qualitative information is obtained which is useful in the subsequent analysis of a complete cascade of airfolls.

Effect of a vortex on flow about a flat plate. - To find the effect of an adjacent potential vortex upon the circulation about a flat plate of chord $L$ in streaming flow, the method used in reference 7 to estimate 
biplane wing interference is extended. The two-dimensional flow about a circular cylinder which by the Joukowski transformation can be altered to the desired. flow is first considered.

Let $\zeta=\xi+1 \eta$ be the complex coordinate in the circular plane and $f(\zeta)$ be the complex potential of the flow before the "disturbance" of the flow by the introduction of the circular cylinder, $|\zeta|=\frac{5}{4}$.

Then after the cylinder is introduced, by the circular theorem of reference 7 , the complex potential is given by

$$
w(\zeta)=f(\zeta)+\bar{f}\left(\frac{L^{2}}{16 \zeta}\right)-\frac{1 \Gamma_{0}}{2 \pi} \log _{e} \zeta
$$

where $\bar{f}$ denotes the complex confugate of $f$ and $\Gamma_{0} / 2 \pi$ is the circulation about the cylinder.

If $\zeta_{0}$ is a stagnation point on the cylinder, $\frac{d W}{d \zeta}=0$ when $\zeta=\zeta_{0}=\left(\frac{L}{4}\right) e^{i \theta_{0}}, \quad \zeta_{0}\left(\bar{\zeta}_{0}\right)=\frac{L^{2}}{16}, \quad \bar{\zeta}_{0}=\frac{L^{2}}{16 \zeta_{0}}, \quad f^{\prime}\left(\zeta_{0}\right)=u_{0}-1 v_{0}$, and $\bar{f}^{\prime}\left(\bar{\zeta}_{0}\right)=u_{0}+1 v_{0}$ where $u$ and $v$ are the velocity components at $\zeta$ in the "undisturbed flow," that is, before the cylinder is introduced. Thus,

$$
\begin{gathered}
\left(\frac{d W}{d \zeta}\right)_{\zeta_{0}}=f^{\prime}\left(\zeta_{0}\right)-\frac{L^{2}}{16 \zeta_{0}^{2}} \bar{f}^{\prime}\left(\frac{L^{2}}{1 \sigma \zeta_{0}}\right)-\frac{i \Gamma_{0}}{2 \pi \zeta_{0}}=0 \\
\frac{\Gamma_{0}}{2 \pi}=-1 \zeta_{0} f^{\prime}\left(\zeta_{0}\right)+1 \bar{\zeta}_{0} \bar{f}^{\prime}\left(\xi_{0}\right) \\
\frac{\Gamma_{0}}{2 \pi}=-1\left(\xi_{0}+1 \eta_{0}\right)\left(u_{0}-1 v_{0}\right)+1\left(\xi_{0}-1 \eta_{0}\right)\left(u_{0}+1 v_{0}\right) \\
\frac{\Gamma_{0}}{2 \pi}=-2\left(\xi v_{0}-\eta_{0} u_{0}\right) \\
21 \zeta_{0}\left[f^{\prime}\left(\zeta_{0}\right)\right] \equiv 21\left(\xi_{0}+1 \eta_{0}\right)\left(u_{0}-1 v_{0}\right) \\
=21\left(\xi_{0} u_{0}+\eta_{0} v_{0}\right)+2\left(\xi_{0} v_{0}-\eta_{0} u_{0}\right)
\end{gathered}
$$


Therefore,

$$
\frac{\Gamma_{0}}{2 \pi}=\left\{-21 \zeta_{0}\left[f^{\prime}\left(\zeta_{0}\right)\right]\right\}_{\text {Real }}
$$

Let $u_{0}+i v_{0} \equiv q e^{i \psi}$, then $u_{0}-i v_{0}=q e^{-i \psi}=f^{\prime}\left(\zeta_{0}\right)$. Substituting into the above equation gives

$$
\begin{gathered}
\frac{\Gamma_{0}}{2 \pi}=-2\left[i \frac{L q}{4} e^{i\left(\theta_{0}-\psi\right)}\right]_{\text {Real }} \\
=2 \frac{I}{4} q \sin \left(\theta_{0}-\psi\right) \\
=2 \frac{I}{4} v_{t}
\end{gathered}
$$

where $v_{t}$ is the velocity tangent to the circle at $\zeta_{0}$ in the undisturbed flow.

To apply the above result to an undisturbed flow consisting of a uniform velocity $W$ at the angle $\beta$ and a potential vortex $\Gamma$ at $\left(r_{1}, \theta_{1}\right)$, one takes the trailing edge of the cylinder to be a stagnation point and places it at the origin; $\Gamma_{\mathrm{O}} / 2 \pi$ gives the circulation required to maintain the Kutta condition, and

$$
\Gamma_{0}=\pi L W \sin \beta+\frac{L \cos \theta_{I}}{2 r_{1}} \Gamma
$$

The equation above indicates that if the Kutta condition is satisfied the loci of the vortex $\Gamma$ for constant cylinder circulation $\Gamma_{0} / 2 \pi$ form a bipolar system of circles. When the Joukowski transformation is applied to the flow, the cylinder becomes a flat plate of chord $I$ and the circles are transformed into curves which pass through the trailing edge of the plate. The dashed curves in figure 6 are the loct of the vortex $\Gamma$ for constant plate circulation $\frac{\Gamma_{0}}{2 \pi}$ which is given by

$$
\Gamma_{0}=\pi L W \sin \beta+n \Gamma
$$

if the Kutta condition is satisfied. The curve for $n=0$ is labeled the neutral line. When the vortex $\Gamma$ is on the neutral line it induces no velocity at the trailing edge of the plate and has no effect on the cylinder circulation. 
The effect of plate camber may be estimated by assuming the plate to be a circular arc which transforms into a circle displaced from the origin as shown in reference 8 and indicated in figure 7 . It can be seen from figure 7 that, if $\theta$ is the camber of the plate and if $\epsilon$ is the displacement of the circle from the origin, then in the notation of figure 7

$$
\begin{gathered}
R-2 \epsilon=R \cos \frac{\theta}{2} \\
2 a=R \sin \frac{\theta}{2}-- \\
\frac{2 a}{\sin \left(\frac{\theta}{2}\right)}-2 \epsilon=\frac{2 a}{\tan \left(\frac{\theta}{2}\right)} \\
\frac{1}{\sin \left(\frac{\theta}{2}\right)}-\frac{1}{\tan \left(\frac{\theta}{2}\right)}=\frac{\epsilon}{a}=-\tan \alpha \\
\alpha=\tan ^{-1}\left[\frac{1}{\sin \left(\frac{\theta}{2}\right)}-\frac{-1}{\tan \left(\frac{\theta}{2}\right)}\right]
\end{gathered}
$$

The solid curve in figure 6 is the neutral line for a plate with camber angle $\theta=30^{\circ}$. The displacement of the neutral line indicates the manner in which the other curves are displaced by the effect of plate camber.

From figure 6 it can be seen that if a vortex $\Gamma$ is in the vicinity of a plate, the plate circulation is greatest when $\Gamma$ is near the trailing edge. Therefore, if a vortex $\Gamma$ is shed from the leading edge of one of a cascade of airfoils, the circulation about the adjacent airfoll should go through a maximum value fust after the vortex passes under its trailing edge. Although this describes the effect of only one shed vortex, figure 6 indicates that this is the dominating effect, since the vortices shed from the other cascade airfoils do not approach the trailing edge of the airfoil in question so closely. The effect of the vorticity shed from the airfoll itself as its circulation changes is neglected also in this simple quasi-steady analysis. However, it appears that one may conclude that there is little tendency for a cascade airfoil to stall until the adjacent shed vortex approaches its trailing edge. Since a certain amount of time is required for the shed vortex to move to such a location, infinite propagation velocity is precluded even though the time required for boundary-layer separation and accumulation of vorticity is neglected. 
This qualitative description of a mechanism by which stalling can propagate along a cascade of airfoils indicates the direction in which the stall will propagate and shows that the velocity of propagation must be of the same order of magnitude as the free-stream velocity. Experimental verification of the description appears in figures 1,2 , and 4.

Trajectory of a shed vortex. - With the effect of a vortex $\Gamma$ on plate circulation as determined in the previous section for quasi-steady flow, it should be possible to estimate the velocity of stall propagation along a blade row if the trajectory of the shed vortex can be calculated and a stall criterion can be established to determine when the succeeding vortex is shed. Although difficulties are encountered which appear to be insurmountable, the attempts made to calculate the shedvortex trajectory are presented briefly so that these difficulties may be pointed out and so that some qualitative information can be derived which is used in the succeeding analysis.

In the first attempt, the cascade is assumed to be represented by an infinite series of potential vortices as described in reference 9 where it was shown that the conjugate complex velocity vector given by the geometry of figure 8 is

$$
u-i v=\frac{i \Gamma}{2 s} e^{i \lambda} \operatorname{coth}\left(\frac{\pi z}{s} e^{i \lambda}\right)
$$

where $z=r e^{i \theta}=x+i y=$ Complex coordinate and $u+i v=$ Complex velocity vector. Values of the real and imaginary parts of the function

$$
e^{i \lambda} \operatorname{coth}\left(\pi e^{i \lambda} \frac{x}{s}\right)-\frac{s}{\pi x}
$$

are tabulated for various values of $\frac{x}{s}$ in reference 9 . By plotting these data into graphs, $(u+i v)$ can be quickly calculated at any point $\mathrm{z}=\mathrm{x}$ in the field near a vortex street at arbitrary angle $\lambda$, with the vortex at the origin missing. If it is assumed that the vortex at the origin is a free vortex, having been shed from its airfoil, its trajectory can be calculated by a stepwise numerical procedure for a flow geometry approximately the same as the experimental geometry of figure 9.

If it is assurned further that blade (a) in figure 8 stalls when the vortex is nearest the corresponding position in figure 9, the calculated velocity of stall propagation is $\frac{V_{p}}{W_{x}}=\frac{20}{11}$. Similar results are obtained when the calculation is made assuming that alternate blades stall simultaneously (which corresponds approximately to the stall-cell 
pattern of figure 9 for 16 stall cells and 27 blades), and the calculated value for $\frac{V_{p}}{W_{x}}$ is $\frac{20}{9}$, about twice the experimental value. It is concluded that this representation of the cascade is too simple to give good numerical answers and that the effect of the presence of a blade after its circulation is shed should be included in calculating the. trajectory of its shed vortex. This effect is to reduce the transport velocity of the vortex when $1 t$ is near the blade so as to give smalier values for $V_{p} / W_{X}$. The neglected boundary-layer time delay further reduces $\mathrm{v}_{\mathrm{p}} / \mathrm{w}_{\mathrm{x}}$.

In reference 10 expressions are derived for the path of a vortex in streaming flow over a flat plate and for the transport velocity of the vortex. It is shown that the path of a vortex near a flat plate is not simply the Joukowski transform of the path in the circular plane but is given by the Routh stream function. For the case of one vortex near a plate with no circulation, a finite-difference method could be used to determine the time required for the vortex to move between. given points on its path. However, difficulty arises from the fact that when the vortex is near the plate, just after being shed, the assumption of potential flow gives very erroneous results. Near the plate, the calculated transport velocity vector of the vortex becomes infinitely large and points in an upstream direction. Therefore, the initial point of the vortex trajectory cannot be taken near the leading edge of the alrfoll, and the calculation must be started from some point away from the airfoil surface. The shape of the trajectory and the time required for the vortex to be convected downstream is found to depend criticaliy upon the initial point chosen. The location of: the initial point and the time required for the vorticity to be shed from the airfoil surface and to accumulate at this point is determined for a special case in reference 5, but the method used appears to be too involved for the problem at hand.

Examination of the expressions derived in reference 10 indicates that near the plate the shed vortex moves downstream more slowly than when it is away from the plate. This information is used in the subsequent anailyis, which circumvents the difficulties mentioned above and which has been devised to deal with the problem of the convection of a series of vortices shed from a cascade of airfoils.

\section{Analysis of a Vortex Flow Model of Stall Propagation}

Derivation of expressions for propagation velocity.- Velocity measurements taken during stall propagation indicate that for a given operating condition or mean flow, the unsteady velocity perturbation translates at constant velocity $\mathrm{V}_{\mathrm{p}}$ along the cascade, and to a first 
approximation, the shape of the velocity profile of the unsteady perturbation is maintained constant. In other analyses, previously mentioned, it was assumed that the profile is constant, and it is also assumed to be constant here, although data which will be presented subsequently indicate that this is not always strictly true. With the approximation that each cascade airfoil sheds a discrete potential vortex $\Gamma$ when it enters the stall cell and another $-\Gamma$ of opposite sign when it leaves the cell, it follows that all the trajectories of vortices (of same sign) shed from the cascade airfoils must be translates of the same curve and separated by the blade specing $s$ along the cascade.

The vortices which have been shed downstream must be arranged in a pattern as shown in figure 10. The dashed lines indicate the trajectories of the vortices shed from two succeeding blades. The $\Gamma$ vortices must lie on a line (b) which eventually becomes straight downstream of the cascade and the $-\Gamma$ vortices must be located along a line (c) which becomes straight and parallel to line (b) downstream of the cascade. The region between lines $(b)$ and $(c)$ is called the stall cell. Iines (b) and (c) are of constant shape in time and move relative to the cascade with the velocity of propagation $V_{p}$.

In a coordinate system which is fixed to the stall cell, the blades move down (fig. 10) with velocity $v_{p}$, and the shed vortices move downstream along the stationary path lines (b) and (c). The flow is steady in time except for the effects due to the finite spacing of blades and shed vortices. It can be seen from equation (I) that these are local effects confined to an area within approximately one blade spacing of the cascade and one vortex spacing of lines (b) and (c). Flow is diverted to each side of the stall cell which has the nature of a thick wave.

After they are far downstream of the cascade, the shed vortices are spaced uniformly by a distance a along lines (b) and (c) and move with transport velocity $V$. The symbol $\varnothing$ is defined as the angle between each street and the cascade axis, and $n$ is defined as the number of vortex spaces between the streets as indicated in figure 10. In figure 11 are indicated the velocity triangles associated with the stall cell. The velocity at any point far downstream of the cascade is $W_{2}$ plus the induced velocity due to the stall cell (which is the velocity induced by two infinite vortex streets).

If the blades stall out completely when they enter the cell, the strength of the shed vortices will be of the order of magnitude

$$
\pm \Gamma=\left(w_{\theta_{I}}-w_{\theta_{2}}\right) s
$$


From reference 11, for a single infinite row of equidistant vortices, each of strength $\Gamma$ (plus clockwise) at distances a apart, with the origin at a vortex and the axis of $x$ along the row, the velocity components induced by the vortices are

$$
\left.\begin{array}{l}
u=\frac{\Gamma}{2 a} \frac{\sinh (2 \pi y / a)}{\cosh (2 \pi y / a)-\cos (2 \pi x / a)} \\
v=\frac{\Gamma}{2 a} \frac{\sin (2 \pi x / a)}{\cosh (2 \pi y / a)-\cos (2 \pi x / a)}
\end{array}\right\}
$$

and one may calculate the maximum value of $v$ for $y=$ Constant $\neq 0$ as follows:

$$
\begin{aligned}
& \frac{\partial v}{\partial x}=\frac{\frac{\Gamma}{2 a} \frac{2 \pi}{a}\left[\left(\cosh \frac{2 \pi y}{a}-\cos \frac{2 \pi x}{a}\right) \cos \frac{2 \pi x}{a}-\sin ^{2} \frac{2 \pi x}{a}\right]}{[\cosh (2 \pi y / a)-\cos (2 \pi x / a)]^{2}} \\
& {[\sin (2 \pi x / a)]^{2}+[\cos (2 \pi x / a)]^{2}=1} \\
& \cosh \frac{2 \pi y}{a} \cos \frac{2 \pi x}{a}-1=0 \\
& \cos \frac{2 \pi x}{a}=\frac{1}{\cosh \frac{2 \pi y}{a}} \\
& \sin \frac{2 \pi x}{a}=\sqrt{1-\cos ^{2} \frac{2 \pi x}{a}} \\
& =\frac{\sqrt{\cosh ^{2} \frac{2 \pi y}{2}-1}}{\cosh \frac{2 \pi y}{a}} \\
& =\tanh \frac{2 \pi y}{a}
\end{aligned}
$$




$$
v_{\max }=\frac{\Gamma}{2 a \sinh (2 \pi y / a)}
$$

Therefore, far downstream, the maximum lateral velocity (normal to Ines (b) and $(c)$ ) that the vortex streets can induce on each other is

$$
v_{\max }=\frac{\Gamma}{2 a \sinh 2 \pi n}
$$

where na is the distance between lines (b) and (c). If $n>I$

$$
v_{\max }<\frac{W_{\theta_{I}}-W_{\theta_{2}}}{500} \frac{s}{a}
$$

and may be neglected.

The basis for taking $n>I$ is derived from the hot-wire data taken downstream of the isolated rotor where it is noted that the cells always cover at least two blade spaces. This appears to be true, in general, for stall cells in rotors. In the interferometer pictures from the circular cascade, figures 12,2 , and 1 , it is noted that $n \cong I$; however, the downstream flow field extends only 2 or 3 blade chords from the circular cascade, so that the inftial assumption of an infinite downstream field is not valid for the circular cascade.

More then a distance a from the vortex streets the longitudinal velocity induced by them may be neglected outside the cell, while inside the cell, from equation (I), $V_{1}=\Gamma / a$.

The resultant transport velocity of each downstream vortex is $V$ as shown in figure 11 . Solving for the velocity of propagation $V_{p}$ from figure 10 gives

$$
\begin{gathered}
v_{p}=W_{\theta_{2}}-\frac{v_{1}}{2} \sin \phi-\left(w_{x_{2}}-\frac{v_{1}}{2} \cos \phi\right) \tan \phi \\
v_{p}=W_{\theta_{2}}-W_{x_{2}} \tan \phi
\end{gathered}
$$

and if $\phi \cong 0, \quad v_{p} \cong w_{\theta_{2}}$.

This same result can be obtained easily by noting the parallelogram in the velocity diagram of figure 11 and solving for the equal and opposite side from $V_{p}$. One advantage of the assumed vortex flow model is 
that $V_{p}$ can be calculated away from the cascade without solving for the detalled nature of the flow in or near the cascade except insofar as this affects the angle $\phi$. It is observed that $v_{p}$ and the angle $\phi$ are uniquely dependent if the downstream velocity $W_{2}$ is given and if $n>1$. The velocity of propagation $v_{p}$ does not depend explicitiy on the physical size or shape of the stali cell or on the magnitude of the velocity perturbation. Since the angle $\phi$ has been observed experimentally to be small in many cases, equation (2) indicates that the fair experimental agreement between $V_{p}$ and $W_{\theta_{2}}$ which has been noted by other observers is an immediate consequence. However, in the analysis to follow, no assumptions regarding the angle $\phi$ are made.

To continue the analysis, the following variables are defined:

$N \equiv \frac{V_{1} \cos \phi}{W_{x_{2}}}$

$P \equiv \Gamma / \Gamma_{0}=\frac{\text { Shed vorticity }}{\text { Bound vorticity }}$

$\mathrm{x} \equiv \frac{\mathrm{W}_{\mathrm{x}_{1}}}{\mathrm{~W}_{\mathrm{x}_{2}}}$

$Y \equiv$ fraction of downstream periphery covered by stall cell, $\frac{\text { na/cos } \emptyset}{2}$

The circulation around a blade away from the cell is given by

$$
\Gamma_{0}=s\left(W_{\theta_{1}}-W_{\theta_{2}}\right)
$$

Then,

$$
V_{1}=\frac{\Gamma}{a}=\frac{P \Gamma_{0}}{a}=P \frac{s}{a}\left(W_{\theta_{1}}-W_{\theta_{2}}\right)=\frac{N W_{x_{2}}}{\cos \phi}
$$

and $\frac{s}{a}=\frac{N}{P\left(X \tan \beta_{1}-\tan \beta_{2}\right) \cos \phi}$. 
The time-average continuity equation gives

$$
\begin{aligned}
& 2 W_{X_{1}}=\left(2-\frac{n a}{\cos \phi}\right) W_{X_{2}}+\frac{n a}{\cos \phi}\left(W_{x_{2}}-V_{1} \cos \phi\right) \\
& X-1=Y(1-N)-Y \\
& X=1-Y N
\end{aligned}
$$

Solving from figure II for the velocity with which the vortices move along lines (b) and (c) gives

$$
v_{a}=\frac{w_{x_{2}}}{\cos \phi}-\frac{v_{1}}{2}
$$

Since the frequency with which vortices pass downstream points on lines (b) and (c) must be the same as the frequency with which vortices are shed onto the lines,

$$
\frac{s}{a}=\frac{v_{p}}{V_{a}}=\frac{w_{\theta_{2}}-w_{x_{2}} \tan \phi}{\frac{W_{x_{2}}}{\cos \phi}-\frac{V_{1}}{2}}=\frac{\left(\tan \beta_{2}-\tan \phi\right) \cos \phi}{1-\frac{N}{2}}
$$

Eliminating s/a from equations (4) and (6) gives

$$
\left(\tan \beta_{2}-\tan \phi\right) \cos ^{2} \phi=\frac{N-\left(N^{2} / 2\right)}{P\left(X \tan \beta_{1}-\tan \beta_{2}\right)} \equiv K
$$

From the velocity diagram for an isolated rotor in figure 13 it can be seen that

$$
x \tan \beta_{1}-\tan \beta_{2}=\tan \alpha_{2}
$$

for an isolated rotor with no upstream whirl velocity $\left(c_{\theta_{1}}=0\right)$.

It is noted that since the shed vortices are convected downstream, $\mathbb{N}<2$. Also, the range of $P$ must be limited to $0<P<1$. The curves of $\mathrm{K}$ versus $\mathrm{N}$ for $\mathrm{P}\left(\mathrm{X}\right.$ tan $\left.\beta_{1}-\tan \beta_{2}\right)$ assumed constant are parabolas. Curves of $K$ versus tan $\phi$ for $\beta_{2}$ assumed constant are almost straight for $\tan \phi>-0.2$, as shown in figure 14, which is a plot of 
$K$ against $\tan \phi$ for $\beta_{2}=30^{\circ}$ and $50^{\circ}$. . (Experimental values of $\beta_{2}$ for the isolated rotor from which data were taken fall between $30^{\circ}$ and $\left.50^{\circ}.\right)$

A diagram of $\mathrm{K}$ plotted against $\mathrm{N}$ and $\mathrm{K}$ plotted against $\tan \varnothing$ with the intercepts and peaks labeled is shown in figure 15 . It is ind1cated that for a given value of $K$ there are either two or zero solutions for the angle $\phi$ (and $v_{p}$ ). However, solutions which are physically possible appear to lie on the positive side of the peak of the curve of $K$ against $\tan \phi,\left(\tan \phi>\tan \beta_{2}-\sec \beta_{2}\right.$ in fig. 15), since experimental values of $\phi$ presented herein fall between $-15^{\circ}$ and $19^{\circ}$ and since all known experimental values of $V_{p}$ are less than $W_{\theta_{I}}$. Qualitative reasoning based on the results given in the section entitled "Effect of a Vortex on Flow About a Flat Plate" indicates that a blade has little tendency to begin to stall untll the vortex shed from the preceding blade approaches the trailing edge. Since time $s / V_{p}$ is required for the vortex to be formed and shed from a blade and to approach the trailing edge of the succeeding blade when it begins to stall, $s / V_{p}$ is also the time required for the vortex to move from points (1) to (2) in figure 10. Since it has been shown that the velocity of the shed vortex is less when it is near the blade than when it is downstream (for an isolated blade and vortex) and since there is a boundary-layer time delay required for the vortex to be formed, it would appear that large negative values for $\phi$ are impossible for typical cascade geometries. This precludes the possibility of infinite propagation velocity, since from equation (2) for $v_{p} \rightarrow \infty, \phi \rightarrow-90^{\circ}$. For the reasons above, only the solution giving the smaller value of $\phi$ and $v_{p}<W_{\theta_{I}}$ (as indicated in $\mathrm{flg}$. 15) will be retained.

Therefore, $\tan \beta_{2}-\sec \beta_{2}<\tan \phi<\tan \beta_{2}$ and $\mathrm{K}$ plotted against $\tan \phi$ can be roughly approximated by a straight line which passes through the intercepts $\tan \beta_{2}, 0$ and $\left(0\right.$, $\left.\tan \beta_{2}\right)$. It can be seen from the geometry of figure 15 that within this approximation

$$
\frac{V_{p}}{W_{X_{2}}} \cong K=\frac{N-\left(N^{2} / 2\right)}{P\left(X \tan \beta_{1}-\tan \beta_{2}\right)}
$$

or, for an isolated rotor, 


$$
\begin{aligned}
& \frac{v_{p}}{W_{x_{2}}} \cong \frac{N-\left(N^{2} / 2\right)}{P \tan \alpha_{2}} \\
& \frac{v_{p}}{C_{\theta_{2}}} \cong \frac{N-\left(N^{2} / 2\right)}{P \tan ^{2} \alpha_{2}}
\end{aligned}
$$

If $N \cong I$, there is small net axial flow through the blades in the stall cell since lines (b) and (c) in flgure 10 are path lines and the mass flow into the cell through the blades must be equal to the axial flow in the cell downstream. It is assumed that the blades in the stall cell must lose most of their circulation if $N \cong I$ and, as a result $P \cong I$.

Experimental values of $N$ appear to be close enough to unity to assume that $N=I$, since the variation of $K$ with $N$ is small for values of $N$ near unity as shown in figure 15 (if $3 / 4<N<1$, then $\left.0.47<\frac{K}{\tan \alpha_{2}}<0.50\right)$

For an isolated rotor $C_{\theta_{I}}=0$ and for $P=\mathbb{N}=I$ equation (8) becomes

$$
\left.\begin{array}{l}
\frac{v_{p}}{w_{x_{2}}} \cong \mathrm{K} \cong \frac{\cot \alpha_{2}}{2} \\
\frac{v_{p}}{c_{\theta_{2}}} \cong \frac{\cot ^{2} \alpha_{2}}{2}
\end{array}\right\}
$$

Equation (9) is based upon the approximation that $K$ plotted against $\tan \phi$ is linear. However, if $P=N=1$, equation ( 7 ) can be solved for tan $\varnothing$ explicitly without recourse to this approximation.

If $\mathrm{P}=\mathrm{N}=1$, equation ( 7 ) becomes

$$
\begin{gathered}
\tan \beta_{2}-\tan \phi=\frac{1+\tan ^{2} \phi}{2\left(x \tan \beta_{1}-\tan \beta_{2}\right)} \\
0=\tan ^{2} \phi+2\left(x \tan \beta_{1}-\tan \beta_{2}\right) \tan \phi+1-2 \tan \beta_{2}\left(x \tan \beta_{1}-\tan \beta_{2}\right)
\end{gathered}
$$




$$
\begin{aligned}
\tan \phi= & -\left(x \tan \beta_{1}-\tan \beta_{2}\right) \pm \\
& {\left.\left[\left(x \tan \beta_{1}-\tan \beta_{2}\right)^{2}-1+2 \tan \beta_{2} \overline{(x} \tan \beta_{1}-\tan \beta_{2}\right)\right]^{1 / 2}-} \\
= & \tan \beta_{2}-x \tan \beta_{1} \pm\left[\left(x \tan \beta_{1}-\tan \beta_{2}\right)\left(x \tan \beta_{1}+\tan \beta_{2}\right)-1\right]^{1 / 2} \\
= & \tan \beta_{2}-x \tan \beta_{1} \pm \sqrt{x^{2} \tan ^{2} \beta_{1}-\tan ^{2} \beta_{2}-1} \\
& \frac{V_{p}}{\bar{W}_{x_{2}}}=\tan \beta_{2}-\tan \phi=x \tan \beta_{1} \mp \sqrt{x^{2} \tan ^{2} \beta_{1}-\tan ^{2} \beta_{2}-1}
\end{aligned}
$$

$$
\frac{V_{p}}{W_{x_{I}}}=\tan \beta_{1} \mp \sqrt{\tan ^{2} \beta_{1}-\frac{\tan ^{2} \beta_{2}+1}{x^{2}}}
$$

The plus sign for the radical in equation (10) is discarded since it implies that $\tan \phi<\tan \beta_{2}-\sec \beta_{2}$ and $v_{p}>w_{\theta_{1}}$.

Therefore,

$$
\frac{v_{p}}{w_{\theta_{1}}}=1-\sqrt{1-\left(\frac{w_{2}}{w_{\theta_{1}}}\right)^{2}}
$$

For an isolated rotor,

$$
\frac{V_{Q}}{U}=\sqrt{1-\left(\frac{W_{2}}{W_{\theta_{1}}}\right)^{2}}
$$

since $W_{\theta_{1}}=U$, and $V_{Q}=U-V_{p}$.

If it is assumed that the flow outside the stall cell is loss free and that an 1sentropic pressure coefficlent $c_{p}$ may be used outside the stall cell, then

$$
c_{p} \equiv \frac{p_{2}-p_{1}}{\frac{1}{2} \rho W_{1}^{2}}=1-\left(\frac{W_{2}}{W_{1}}\right)^{2}<\sin ^{2} \beta_{1}
$$




$$
\begin{gathered}
C_{p}=I-\left(\frac{W_{x_{2}} \cos \beta_{1}}{W_{x_{I}} \cos \beta_{2}}\right)^{2}=1-\frac{1}{x^{2}} \frac{1+\tan ^{2} \beta_{2}}{1+\tan ^{2} \beta_{1}} \\
x^{2}\left(I-C_{p}\right)=\frac{I+\tan ^{2} \beta_{2}}{I+\tan ^{2} \beta_{1}}
\end{gathered}
$$

Therefore, substituting in equation (10) gives

$$
\begin{aligned}
& \frac{V_{p}}{W_{X_{I}}}=\tan \beta_{1}-\sqrt{\tan ^{2} \beta_{1}-\left(I+\tan ^{2} \beta_{1}\right)\left(I-c_{p}\right)} \\
& =\tan \beta_{I}-\sqrt{\tan ^{2} \beta_{I}-\left(1-C_{p}\right)-\tan ^{2} \beta_{I}+c_{p} \tan ^{2} \beta_{I}} \\
& =\tan \beta_{I}-\sqrt{\frac{C_{p}}{\cos ^{2} \beta_{I}}-I} \\
& \frac{V_{p}}{W_{x_{1}}}=\tan \beta_{1}-\sqrt{\frac{p_{2}-p_{1}}{\frac{1}{2} \rho W_{x_{1}}^{2}}-I}
\end{aligned}
$$

Equations (11) and (I2) indicate that for stall cells of the type assumed $\left(P=M=I\right.$ and $\left.0<V_{p}<W_{\theta_{1}}\right)$ real values of $v_{p}$ are possible only if $W_{2}<W_{\theta_{1}}, C_{p}>\cos ^{2} \beta_{1}$, and $\beta_{1}>45^{\circ}$.

If $W_{2}=W_{\theta_{I}}$, then $v_{p}=W_{\theta_{1}}$, tan $\phi=\tan \beta_{2}-\sec \beta_{2}$, as indicated in figure 15 , and $c_{p}=\cos ^{2} \beta_{I}$. It is interesting to note that this corresponds to the propagation velocity predicted in reference 4 for a simplified cascade geometry and an entirely different type of stall cell, where the upstream velocity perturbation was assumed to be a sine wave of small amplitude and many blade chords wave length. In reference 4, for this type of cell, it was shown that

$$
\frac{v_{p}}{W_{x_{I}}}=\frac{2\left(I-C_{p}\right)}{\sin 2 \beta_{I}}
$$


$c_{p}=\cos ^{2} \beta_{1}$,

$$
\frac{v_{p}}{W_{x_{1}}}=\frac{2 \sin ^{2} \beta_{1}}{2 \sin \beta_{1} \cos \beta_{1}}=\tan \beta_{1}
$$

where $V_{p}=W_{\theta_{I}}$. The preceding analysis indicates that this is a limiting value and that, for the assumed type of finite-amplitude stall cells to exist, $C_{p}>\cos ^{2} \beta_{1}$.

It is assumed in the preceding analysis that all the downstream circulation is about concentrated vortices. The implication is that the wakes shed from the blades outside the cell are thin enough or $\mathrm{mix}$ soon enough to be neglected and that this is also true for the blade wakes in the cell (or that the velocity through the blades in the cell Is small enough for the vorticity shed inside the cell to be neglected). The assumption of $P=1$, together with that above, Implies that the blades outside the cell have no drag and those inside the cell have no lift. It is assumed that the analysis is valid for more than one cell if they are spaced far enough apart so that equation (3) remains valid, since the cells induce no velocity on each other except for their blockage effect on the main flow which is considered in the analysis.

Since in a coordinate system fixed to the stall-cell pattern of figure 10, the flow is steady and the streamlines are straight and parallel downstream, the static pressure is constant downstream. There is a "head loss" in the stall cell determined by the veloclty defect there, just as in the case of a bluff body wäke; and the fraction of the downstream periphery covered by stall cells could be expressed by a time average loss coefficient of stagnation pressure. The qualitative nature of the flow for $(P=N=I)$ is similar to the flow about a flat plate translating along the cascade at velocity $V_{p}$ and blocking the flow through a portion of it.

\section{Analysis of Model for Small Perturbations}

It is noted that equation (7) gives a relationship between the three parameters $(P, N$, and $\phi)$ and was developed without any assumptions regarding blade stall characteristics other than the assumption of discrete vortices downstream. If another independent equation can be derived between $P, N$, and $\phi$, by specifying the dynamic blade stall characteristics, $V_{p}$ can be determined as a function of $N$ only or of the stall-cell amplitude. 
For small perturbations, $M$ and $N$ can be related to lift and drag coefficients since for an isolated airfoil

$$
\text { Lift }=\rho W \Gamma_{0}=\frac{1}{2} \rho W^{2} I C_{L}
$$

and

$$
\Gamma_{0}=\frac{C_{L} W}{2} L
$$

The assumption is made, as in reference 3, that also for a cascade of airfolls $\Gamma_{0}=\frac{C_{L} W}{2} L$, where $W$ is the local mean velocity and $\Gamma_{0}$ is the bound vorticity at an airfoil. If $C_{I}$ is a known function of local mean air angle $\beta$ as determined by the local incidence, $M(N, \phi)$ can be determined independently of equation (7).

It is difficult to determine $W$ and $\beta$ at the airfoils near the stall cell for arbitrary values of $N$ because the shape of lines (b) and (c) in figure 10 must be known. However, if $N \ll 1$, lines (b) and (c) must be straight even near the cascade, and it becomes possible to determine $W$ and $\beta$ by restricting the amplitude of the stall cell to a small perturbation.

For the velocity induced by a straight vortex street of finite length, equation ( 1 ) indicates that, except in the immediate vicinity of the street, it may be considered as a distributed sheet of strength per unit length $\frac{\Gamma}{a}$; therefore, with the notation of figure 16, the velocity components induced by an element of length $\mathrm{dx}$ are

$$
\begin{aligned}
& d u=-\frac{\Gamma}{2 \pi a} \frac{d x}{r} \sin \theta \\
& d v=\frac{\Gamma}{2 \pi a} \frac{d x}{r} \cos \theta
\end{aligned}
$$

For a sheet of finite length, as in figure 17,

$$
u=-\frac{\Gamma}{2 \pi a} \int_{x_{1}}^{x_{2}} \frac{y d x}{x^{2}+y^{2}}=\frac{\Gamma}{2 \pi a}\left(\tan ^{-1} \frac{x_{1}}{y}-\tan ^{-1} \frac{x_{2}}{y}\right)
$$




$$
\begin{aligned}
& v=\frac{\Gamma}{2 \pi a} \int_{x_{1}}^{x_{2}} \frac{x d x}{x^{2}+y^{2}}=\frac{\Gamma}{4 \pi a}\left[\log _{e}\left(x_{2} 2+y^{2}\right)-\log _{e}\left(x_{1} 2+y^{2}\right)\right] \\
& u=\frac{-\Gamma}{2 \pi a} \delta \\
& \left.\mathrm{v}=\frac{\Gamma}{2 \pi a} \log _{e} \frac{r_{2}}{r_{1}}\right\}
\end{aligned}
$$

It follows that the velocity induced at the blade in the center of the stall cell $V_{1}$, as shown in flgure 18, is parallel to the streets and equal to $\frac{\Gamma}{2 a}$ if $m \gg n$. For various assumptions of $C_{L}(\beta)$, it might be assumed that the blades in the stall cell have a circulation corresponding to $W$ and $\beta$ at the blade in the center of the cell and $V_{p}$ can be calculated as a function of $N$.

However, values of $P$ whlch correspond to values of $N$ as determined by the dynamic stall characteristics of the airfoll restrict the curve of $\mathrm{K}$ against $\mathrm{N}$ to an "operating line" similar to the dashed curve of figure 15. As $N \rightarrow 0, P \rightarrow 0$ and equation (8) becomes indeterminate. However, if it is assumed that $\phi$ must remain small as $N \rightarrow 0$ for the reasons given previously, then from equation $(7) \quad P \cong 2 N \ll 1$. It appears, therefore, that for small perturbations, and small angles $\phi$, the percentage of vorticity the airfolls shed upon entering a stall cell must be approximately equal to the percentage of downstream axial velocity defect in the stall cell. Therefore, the reason that smallperturbation sta.11 cells are not commonly observed may be that a compressor blade generally is thin, stalls from its leading edge during stall propagation, and must shed a large percentage of 1 ts bound vorticity upon stalling. This description appears to apply to the dynamic stall characteristics of the airfoils used in the experiments although in quasi-steady flow the airfoils exhibit a continuous curve of $C_{L}$ against $i$ even in stall as indicated by figures 19 to 22 . It is shown in the section entitled "Determination of Flow Properties From Interferograms" that the airfolls display stall characteristics during stall propagation which depart considerably from their quasi-steady stall characteristics. It is felt that the sudden increase of $i$ as the airfoils enter a stall cell causes them to stall abruptly from their leading edges for the reason given in the section entitled "Correlation of Analytical and Experimental Results," even though they stall "gently" with a slow (quasi-steady) increase of 1 . 
A qualitative reason that a compressor blade should not shed a small vortex from its leading edge during stall propagation is indicated in reference 5 where it is shown that, for a flat plate moved impulsively from rest normal to its plane, vortices must form at the edges of the plate (if infinite velocity is precluded) and that, during the initial formation of these edge vortices, similitude of the flow requires them to grow until they are not small in proportion to the width of the plate. Of course, the stronger the vortex shed from a cascade blade, the greater is the tendency for the adjacent blade to stall as the vortex moves past it and the greater is the tendency for stall propagation; however, there is no apparent reason why propagation of small perturbations should not occur except that this appears to be precluded by the dynamic stall characteristics of the airfoils.

Since small-perturbation stall cells which are two-dimensional (hub to tip in a rotor) and stable have never been observed experimentally to the knowledge of the author, the analysis is pursued no further. The discussion above is offered as a qualitative speculation (not an attempt to prove) that for two-dimensional stall cells large perturbations are more likely to occur as a stable flow pattern then small perturbations.

Prediction of a trend for the number of cells.- The vortex-flow model used in the analys is appears to offer an explanation for the general trend toward larger numbers of cells with increasing throttling of flow as indicated by the experimental data. The experimental results indicate that with further throttling of the flow through an isolated rotor after the formation of one stall cell the cell first grew wider ( $n$ increased in $\mathrm{fig}$. IO), then two cells appeared and grew wider, and so forth until four cells appeared and surging flow began. The vortex flow model Indicates that if $m$ is finite in figure 18, the velocity induced by the stall cell at the blade in the center of the cell, $\left(v_{i}\right)_{c}$, decreases as $\mathrm{n} / \mathrm{m}$ increases. This effect can be shown qualitatively by assuming the lines (b) and (c) to be entirely straight. Then from equation (13), if $m \gg n$

$$
\left(v_{1}\right)_{c}=\frac{\Gamma}{a}\left(\frac{1}{2}-\frac{n}{2 \pi m}\right)
$$

As $n / m$ increases, $\left(V_{1}\right)_{c}$ decreases faster than near the edges of the cell, there is more flow through the center of the cell than near the edges, and the blade at the center of the cell tends to unstall, thereby splitting the cell into two cells which become distributed axisymetrically around the rotor.

It does not appear possible to predict when a stall cell will split as $\mathrm{n} / \mathrm{m}$ increases without detalled information regarding the dynamic stall-unstall characteristics of the airfoils and the shape of the cell 
(Iines (b) and (c) in fig. 10). The estimated relationship between $\mathrm{n} / \mathrm{m}$ and $\left(v_{j}\right)_{c}$ does not indicate much effect on $\left(v_{i}\right)_{c}$ until $\mathrm{n} / \mathrm{m}>1 / 3$. The measured effect is much greater and is described subsequently.

\section{Summary of Analysis}

By making use of experimental data in order to develop a simplified analytical flow model, a theory of stall propagation in an isolated blade row has been developed which appears to be based upon fewer restrictive assumptions than some of the earlier analyses. In the analysis of the present paper, a restriction is imposed upon the nature of the stall cells. Although the approximation to the actual observed flows appears to be valid for all the blading, geometrical configurations, and flow conditions for which the experiments described herein were run, it is unknown how valid the approximation is in general. It is speculated that the approximation regarding the nature of the cells might be widely applicable to continuous cascades of thin airfoils where the flow extends many blade chords downstream of the cascade.

It should be noted that in the preceding analysis no proof of existence, equilibrium, or stability is given for the assumed flow model. The experimental data are offered in lieu of mathematical proof.

EXPERIMENTAL INVESTICATION OF ROTATING STAIL

\section{Investigation in a Circular Cascade}

Description of apparatus and procedure. - With the sponsorship of the NACA, a circular radial-outflow cascade was designed and installed in the closed-circuit wind tunnel at the Ges Turbine Laboratory during 1953. The cascade was designed specifically for investigation of rotating stall and is described in detail in reference 12. In figure 23 is shown a schematic view of the test section which includes a ring of variableangle guide vanes with which the air inlet angle to the test cascade $\beta_{I}$ can be continuously varied. Some dimensions of the test cascade are:

Radius to guide vane trailing edges

(varies with $\beta_{I}$ ), in. ........... 7.2 to 6.7

Radius to blade leading edges and measuring stations 1

and 2 , respectively:

For configuration A, 1n. . . . . . . . . 7.91, 7.69, 8.94

For configuration B, in. ......... 8.66, 8.44, 9.69 
Blade chord, in. . . . . . . . . . . . . . . 0.96

Blade span, in. . . . . . . . . . . . . . . 1.71

Blade profile NACA 65(12)10 as changed by circular transformation

Number of blades................. 54, 27, 18, 9 Nominal solidities, $\sigma$. . . . . . . . . . $1,1 / 2,1 / 3,1 / 6$

Simulated Iinear cascade $65(8) 10$

Mean line a...................... 1.0

Some advantages of the cascade geometry are:

(1) The flow is two-dimensional through the cascade neglecting the wall boundary layers.

(2) Optical measurement of the flow through a portion of the cascade is possible.

(3) A continuously rotating stall pattern can be established, which is not possible for a finite-length linear cascade.

Some disadvantages are:

(1) Because of the radial flow, the pressure distribution about the airfoils is not precisely the same for a given pressure rise through the cascade as in a rotor or Iinear cascade. As described in reference 12, a correction was made for this effect by designing the cascade through the conformal transformation of one linear cascade so that the adverse pressure gradient on the suction side of the airfoils in the transformed circular cascade was approximately equal to that for a second Iinear cascade which was simulated. It was found that the circular transformation of a Iinear NACA 65(12)10 cascade gave approximately the same adverse pressure gradient on the circular cascade blades at the design point as was obtained for a linear NACA 65(8)10 cascade. The data for the linearcascade pressure distributions were taken from reference 13. The degree of success of this procedure is indicated in the section "Determination of Flow Properties From Interferograms" where the pressure distribution about the circular-cascade blades is calculated and compared with the distributions from reference 13 for one value of $i$.

(2) The flow field downstream of the cascade is necessarily rather short which appears to have a pronounced effect on the rotating stall cell pattern as discussed in the section "Correlation of Analytical and Experimental Results." 
In reference 4, an experimental investigation of rotating stall in the circular cascade 1 s described, which was conducted to determine the detailed nature of the flow and to ascertain the effects of Mach number, Reynolds number, mean incidence, and cascade solidity on the propagation of stall cells in the cascade. The experimental work described herein is a continuation of this investigation. The procedures and instrumentation are largely the same as those described in reference 4 except for the use of the Gas Turbine Laboratory portable Mach-Zehnder interferometer and a piezoelectric crystal pressure pickup described subsequently.

The purpose of the present Investigation is to extend the data of reference 4 to include the effects of lower cascade stagger angle, of higher mean incidence to the cascade, and of increased clearance between the guide vanes and test cascade. Quantitative measurement of the pressure field in a portion of the cascade during stall propagation is also attempted by means of high-frequency interferometer pictures.

All of the tests in reference 4 were made with a cascade stagger angle $\lambda$ fixed at $43^{\circ}$. The highest angle to which the guide vanes could be turned gave an entering air angle $B_{1}$ to the cascade of $69^{\circ}$; therefore, the highest mean-flow incidence angle 1 possible was $26^{\circ}$. Stall propagation started at $1=20^{\circ}$. In reference 14 , it was observed that stall propagation existed in a rotor-plus-guide-vane axial compressor stage for $34^{\circ}<1<47^{\circ}$ (relative to the rotor at mean radius). Therefore, it was deemed desirable to alter the linkages to the guide vanes to permit greater incidences to the circular cascade. It was noted in the schlieren pictures presented in reference 4 that, during stall propagation in the circular cascade, when an airfoll stalled some of the fluid from the separated boundary layer was washed around the leading edge of the succeeding airfoil before it stalled. In hope of decreasing this effect and in order to increase further the incidence on the cascade airfoils and to observe any other effects on the nature of the flow, the atagger angle of the cascade was changed from $43^{\circ}$ to $31^{\circ}$. For the altered guide-vane linkages and reduced stagiger angle, the range of 1 was from $13^{\circ}$ to $53^{\circ}$. With these two alterations, the following data, similar to those presented in reference 4, were taken at the reduced stagger angle and over a greater range of incidence during stall propegation.

Throughout the investigation in a circular cascade, $C_{p}$ is defined as the ratio of time-average static pressure rise through the cascade to the dynamic pressure of the flow entering $\frac{p_{2}-p_{1}}{\frac{1}{2} p_{1} w_{1}^{2}}$, where $p_{2}$ and $p_{1}$ are wall static pressures as measured by the average of three equally 
spaced taps at measuring station 2 and three at station 1 . It was determined that each of the boundary layers on the cascade walls was less than $1 / 8$ inch thick before the onset of stall propagation and they are neglected henceforth.

In all the series of schlieren and interferometer photographs such as figures 1 and 2 , time is from right to left. Flow is from left to right in each frame. All the schlieren photographs were taken at 5,000 per second and ali the interferometer photographs at 6,000 per second.

Experimental results - configuration A.- Pressure coefficient $C_{p}$ as a function of $\beta_{1}$ for cascade solidity of unity is shown in figure 19 for two levels of Reynolds number $R e_{l}$. The conventional $\left(C_{p}\right)_{\max }$ is at $\beta_{1}=46^{\circ}$ in figure 19. Subsequent hot-wire data and schlieren pictures indicate that periodic stall propagation occurred at the peaks in the curve where $\beta_{1}$ was $540,62^{\circ}$, and $68^{\circ}$ and where values of $\beta_{1}$ were greater than $79^{\circ}$. On each side of the peaks the stall cells gradually became intermittent and of lower amplitude, and between the peaks the flow was relatively steady in time. Visual observation of the flow through the schlieren apparatus for values of $\beta_{1}$ between the peaks of figure 19 showed that the flow was completely separated from the suction side of the airfoils.

As $\beta_{I}$ was varied, the mass flow through the test section was held approximately constant; therefore, Reynolds number and Mach number increase with $\beta_{I}$ for each curve plotted, as indicated in figure 19.

It is noted in figure 19 that there was a distinct effect on $C_{p}$ caused by change of Reynolds number for $58^{\circ}<\beta_{1}<74^{\circ}$. At the lower Reynolds numbers propagation no longer occurred at the band of $\beta_{1}$ centered at $62^{\circ}$ and the band at $68^{\circ}$ became narrower. When the Reynolds. number was decreased to 50,000 both of these bands disappeared, but propagation at the $54^{\circ}$ band became more violent and periodic, and the band at $79^{\circ}$ was not affected noticeably.

Schlieren photographs of the flow were taken in each of the bands of $\beta_{1}$ for which there was stall propagation. The same equipment was used as in reference 4 with the 5-inch portable schlieren apparatus of the Gas Turbine Laboratory altered to use an Edgerton, Germeshausen, and Grier type 501 stroboscopic light source and a General Radio Corporation 35-millimeter camera. Sections of each of these four films are shown in figure 24 .

The velocity of propagation and the number of cells in each band of $\beta_{1}$ were determined from these films_and hot-wire velocity measurements 
made upstream of the cascade at a radius of 7.69 inches. These data are presented in figure 25 together with values of $\mathrm{V}_{\mathrm{p}} / \mathrm{C}_{r_{I}}$ predicted for a circular cascade by the equation on page 42 of reference 4. (Values for $\beta_{2}$ used in this equation were determined by extrapolation from the values measured in schlieren photographs before the onset of stall propagation as was done in ref. 4.)

Traverses of the hot-wire probe across the span of the cascade airfolls indicated that the flow was two-dimensional for all the bands except for values of $\beta_{1}$ greater than $79^{\circ}$ where the velocity fluctuations were about twice as great near the walls as in the center of the blade span. Typical hot-wire traces taken at $r=7.69$ inches for the four bands of propagation are shown in figure 26. The hot-wire equipment used was a Flow Corporation model HWB hot-wire anemometer and another similar unit with hot wires of 0.003 -millimeter diameter and $0.04-1$ nch length.

By careful examination of the negatives from which figure 24 was made, one can discern the wakes from the upstream guide vanes. It was attempted to make one wake more visible by heating a turning vane with soldering iron filaments brazed to each end. However, no effect could be noticed in the pictures. In figure $24(\mathrm{a})$, during the first band of $\beta_{1}$ for which there was propagation, neither these wakes nor the fluid from the adjacent separated airfoils were washed over the leading edges of the airfoils during stall propagation. In flgure 24 (b), a turningvane wake just barely flicked over the leading edge of each blade before it stalled and not much of the separated fluid from the adjacent blade was washed over. In figure $24(c)$, the same wake as in figure $24(b)$ crossed back and forth over the leading edge. of each blade and more of the separated fluid was washed over. In figure 24(d), the wakes cannot be detected. It is probable that the wakes and separated fluid injected Into the blade boundary layers had an effect on the time required for the blades to stall. This may have caused the great increase of propagation velocity from figures $24(a)$ to $24(b)$. This effect is more evident when $V_{p} / C_{\theta_{I}}$ is calculated rather than $V_{p} / C_{r_{I}}$. For the bands in order of increasing values of $\beta_{1}, v_{p} / C_{\theta_{1}}$ was $0.13,0.28$, and 0.25 , Thus, $\mathrm{V}_{\mathrm{p}} / \mathrm{C}_{\theta_{1}}$ increased by a factor of 2 from the first to the second band and then remained virtually constant.

Because the stall cells were not all precisely the same size and shape for a given mean flow, there is an uncertainty in measuring $V_{p}$ and the number of cells. It is belleved that the uncertainty in determining the number of cells was less than five for the first band, three for the second and third, and zero for the last band at $71^{\circ}$. The 
uncertainty in $V_{p}$ is about 12 percent for the last band, 8 percent for the first, and less than this for the other two as indicated in figure 25 .

When half the blades were removed from the cascade, this being the only alteration, the following experimental results were obtained for configuration $A$, at a value of $\sigma=1 / 2$. Pressure coefficient $C_{p}$ is plotted against $\beta_{1}$ in figure 19 so that comparison can be made with the data for $\sigma$ of unity. It is observed that for $\sigma=1 / 2$ there was a mean pressure drop through the cascade for some values of $\beta_{I}$. Hotwire traces showed that the first band of $\beta_{I}$ for which there was stall propagation was at 540 and was very narrow. The signals were never periodic but were intermittent with occasional lapses to high-frequency turbulence. For $60^{\circ}<\beta_{1}<64^{\circ}$ a periodic signal was observed as shown in the photograph designated $\mathrm{E}$ in figure 26 . For $64^{\circ}<\beta_{1}<79^{\circ}$ there were low-amplitude unperiodic fluctuations in the flow which became more distinct for $\beta_{1}<79^{\circ}$. Traverses of the hot wire indicated that the velocity fluctuations were two-dimensional and periodic only for $60^{\circ}<\beta_{1}<64^{\circ}$. In the $54^{\circ}$ band, velocity fluctuations existed only in the center third of the blade span and for $\beta_{1}<79^{\circ}$ only in outer thirds of the span near the walls.

Schileren pictures were taken for $\beta=60^{\circ}$ and a section of the film is shown in figure 9. From the film and hot-wire traces the number of cells and their velocity of propagation were determined and are indicated in figure 25. In figure 9, the wakes from two of the guide vanes are visible. (They have been darkened to aid in reproduction.) From the motion of these wakes it is apparent that the unsteady fluctuations in the flow are large. It is observed that the wakes briefly cross over the leading edges of the cascade blades as they fluctuate. In figures 24 and 9 , the boundary layer appears to separate from near the leading edges of the alrfoils when they stall and to reattach when they unstall.

When the Reynolds number was lowered below 50,000 for a value of $\sigma=1 / 2$, the velocity fluctuations became nonperiodic and of very low amplitude for all the bands of $\beta_{1}$ where stall propagation had existed at higher Reynolds numbers.

In figure 12 is seen a section of an interferometer film taken for configuration $A$ with $\sigma=I / 2$ and $\beta_{I}=60^{\circ} ; R e_{1}$ and $M_{I}$ are 333,000 and 0.47 , respectively. The bands in figure 12 indicate lines of constant mean air density, which correspond to lines of constant pressure if isentropic flow can be assumed and lines of constant velocity magnitude also where the time rate of change of velocity can be neglected. 
The striking feature of figure 12 is the distinct appearance of the vortices shed from the leading and trailing edges of the airfoils.

Experimental results - configuration B. - To determine the effect of interference between guide vanes and test cascade, the radil of the test cascade and the static pressure taps for measuring $p_{1}$ and $p_{2}$ were increased three-quarters of an inch to configuration $B$. This effectively doubled the clearance between the blade rows and shifted their relative angular position approximately $3^{\circ}$ about the axis of the test section.

In figure $20 \quad C_{p}$ is plotted against $\beta_{1}$ for configuration $B$ and $\sigma=1$. Comparison with the corresponding $C_{p}$ curve for configuration $A$ from figure 19 indicates that the Increased clearance had no pronounced qualitative effect upon $C_{p}$, although values of $C_{p}$ were generally greater for configuration $B$. The bands of $\beta_{1}$ where stall propagation existed were shtfted a few degrees, which was probably caused by the angular shift of the blade rows from configurations $A$ to $B$ through the effect of the turning vane wakes on the dynamic stall characteristics of the cascade airfoils. There were also detalled effects on the flow caused by the increased clearance. Periodic two-dimensional stall propagation existed at $\beta_{1}=57^{\circ}$ and $63^{\circ}$. Low-amplitude, low-frequency, unperiodic fluctuations existed for $50^{\circ}<\beta_{1}<53^{\circ}$ and for $\beta_{I}>78^{\circ}$. Flow was relatively steady between the peaks of $\beta_{I}$ in figure 20 , as was described for figure 19. Figure 27 shows three interferograms of the flow at incidences where the flow was comparatively steady. Interferometer pictures were taken at $\beta_{1}=57^{\circ}$ and $64^{\circ}$ and are presented in in figure 1 . The number of stall cells and their velocity of propagation as determined from these pictures are shown in figure 20.

When the cascade was assembled with 27 blades $(\sigma=1 / 2)$, it was found that stall propagation exlsted for $53^{\circ}<\beta_{1}<68^{\circ}$. However, the perturbations were two-dimensional and periodic only for $56^{\circ}<\beta_{1}<66^{\circ}$. It was found that decreasing the Reynolds number made the fluctuations less periodic and of lower amplitude. Interferometer pictures were taken for $\beta_{1}=580,61^{\circ}$, and $64^{\circ}$ and are presented in figures $2(a)$ to 2(c). (The pictures for $\beta_{1}=61^{\circ}$ are used to calculate the pressure field during one cycle of the motion described in the following section.) The following data apply to figure 2: 


\begin{tabular}{|c|c|r|r|r|c|c|c|}
\hline Figure & $\beta_{I}$, deg & $\begin{array}{r}\text { Number of } \\
\text { cells }\end{array}$ & $V_{\mathrm{p}} / C_{r_{I}}$ & $V_{p} / C_{\theta_{I}}$ & $C_{p}$ & $R_{I}$ & $M_{I}$ \\
\hline $2(a)$ & 58 & 15 to 16 & 0.78 & 0.42 & 0.25 & 266,000 & 0.30 \\
$2(b)$ & 61 & 19 to 20 & .87 & .43 & .15 & 235,000 & .37 \\
$2(c)$ & 64 & 14 to 15 & 1.00 & .45 & .13 & 200,000 & .31 \\
\hline
\end{tabular}

The cascade was reassembled with 18 blades $(\sigma=1 / 3)$ and it was found that periodic two-dimensional stall propagation existed only for narrow bands of $\beta_{1}$ about $57^{\circ}$ and $70^{\circ}$. Interferometer pictures at these two values of $\beta_{1}$ are shown in figure 3 and the following data apply to them:

\begin{tabular}{|c|c|c|c|c|c|c|c|}
\hline Figure & $\beta_{1}$, deg & $\begin{array}{c}\text { Number of } \\
\text { cells }\end{array}$ & $v_{p} / C_{r_{1}}$ & $v_{p} / C_{\theta_{1}}$ & $C_{p}$ & $R_{1}$ & $M_{1}$ \\
\hline $3(a)$ & 57 & 7 to 8 & 1.27 & 0.83 & 0.15 & 255,000 & 0.31 \\
$3(b)$ & 70 & 18 & 1.47 & .91 & .058 & 264,000 & .32 \\
\hline
\end{tabular}

When the cascade was assembled with nine blades $(\sigma=1 / 6)$, it was found that hot-wire traces upstream of the cascade indicated only varying-amplitude high-frequency velocity fluctuations as $\beta_{I}$ was increased. At $\beta_{I}=57^{\circ}$, however, the fluctuations appeared to be intermittently periodic and interferometer pictures were taken as shown in figure 4. The frequency with which the blade stalls in figure 4 is approximately $790 \mathrm{cps}, \mathrm{Re}_{1}=250,000$, and $\mathrm{W}_{1}=292 \mathrm{ft} / \mathrm{sec}$.

In reference 6 is described an experimental investigation of the Kármán vortex shedding of a flat plate at high incidence 1 . It was found that for $20^{\circ}<i<60^{\circ}$

$$
0.164<\frac{\mathrm{FL} \sin i}{W}<0.150
$$

where $F$ is the frequency at which vortices were shed from one edge of the plate. The result of calculating $\frac{F I \sin 1}{W}$ from the preceding data for configuration $B$ and plotting it against $\sigma$ is shown in figure 5 . Although there is considerable scatter in the data, it is observed that, as was shown by a similax plot for $\lambda=43^{\circ}$ in reference 4 , the frequency 
with which a blade stalls during stall propagation in the cascade appears to approach the Kármán vortex shedding frequency of the 1solated flat plate as the solidity of the cascade is decreased.

Determination of flow properties from interferograms.- From interferometer photographs of a two-dimensional flow, it is possible to determine the pressure field by conventional procedures. The purpose here is to determine the pressure distribution around the circular-cascade airfoils and to estimate the strength of the shed vortices during stall propagation. Also, the pressure distribution before the onset of stall propagation in a circular cascade is compared with that of the simulated linear cascade.

The use of interferometry to obtain quantitative information about two-dimensional pressure fields which are unsteady in time is no more difficult in principle than it is for steadj flows, but practical difficulties are encountered in the photography and direct pressure measurement required. As used herein, the properly adjusted interferometer produces fringes or bands, such as those in figure 2, which indicate lines of constant density. No indication is given in the interferogram of the density level but only of the absolute value of the density differences between fringes. The sign of the density change between fringes 1s determined from the schlieren plctures and from a qualitative knowledge of the flow. It is necessary to determine independently the value of density at some point in the interferogram at the instant the picture is taken. This is done by the determination of two other independent fluid properties at the point which are pressure and entropy. The entropy is assumed constant everywhere in the interferogram (except in the cores of the vortices) and is evaluated upstream of the cascade where the flow is relatively steady and fluid properties can be measured conveniently. The static pressure fluctuations with time are measured at a point in the interferogram with a miniature crystal pressure pickup.

Interferometer: A portable Mach-Zehnder interferometer w1th 5-inchdiameter optical elements was used in this study. A detailed discussion of the design and operation of this instrument is given in reference 15 . Only brief mention of its use will be made here with special reference to the present application. Apertures of $1 / 16$ - and 3/32-inch diameter were used at the focal point of the collimating lens causing the light to emerge from the lens as a parallel beam. At the first splitting plate the beam is divided into two halves which pass respectively through and around the test section before belng recombined at the second splitting plate.- The fact that one beam does not traverse the testsection window is compensated for by including in 1ts path two glass plates which are optically similar to those of the test section. The resulting beam is focused on the film. The Image produced on the film depends on a comparison between the two optical path lengths. 
With no flow in the test section and a small angular displacement between the splitting plates, a serles of parallel interference fringes results. If these bands are originally spaced infinitely far apart, by setting the mirrors exactly parallel, any fringes which appear in the flow picture will be lines of constant density. Some loss of accuracy results from imperfections in the optical elements precluding infinite band spacing. The interferometer optics, test-section windows, and compensating plates combined gave a minimum of one fringe instead of the zero number of fringes implied by perfect optics and exactly parallel mirrors.

A concentrated light source is required to expose the film. The source used was an experimental xenon-filled lamp, a photograph of which is shown in figure 28. It is capable of flashing for approximately one microsecond at rates of 60 to 8,000 per second when used with the Edgerton, Germeshausen, and Grier type 501 stroboscopic modulator: Its small size, large amount of light flux, reliability, and long life make it very satisfactory. The light was filtered to give the monochromatic light necessary to the interferogram evaluation using an interference filter which passed a band of wavelengths 100 angstroms wide centered at 4,500 angstroms.

Pressure measurement: A miniature crystal pickup capable of sensing high-amplitude, high-frequency pressure fluctuations is shown in figure 29. It is a piezoelectric, barium-titanite crystal in the shape of a hollow cylinder 0.12 inch long by 0.09 inch outside diameter. It is insertad in a drilled hole in one of the optical flats at a measuring point midway between two airfoils shown in figure $2(b)$. It is mounted flush with the inner surface and senses the wall static pressure. A drop of glue in the end of the cylinder acts as a safety valve and seals the crystal so that, when the test-section pressure is applied to its outside surfaces, hoop tension stresses are produced which cause mechenical strains and the accumulation of electrical charge on its inner and outer cylinorical surfaces; a voltage results which is sent through a high-impedance amplifier and displeyed on an oscilloscope. Since the impedance of the circuit is not infinite, the charge produced on the crystal faces "leaks off" which prevents its use in measuring lowfrequency pressure fluctuations.

To determine the rise time of the pickup to a step change in pressure, the pickup was mounted in the wall of a shock tube which is described in reference 16. The rise time was less than 0.05 millisecond, very adequate for the intended use. The pickup may be calibrated by determining its sensitivity to temperature and observing how quickly electrical charge leaked from the faces of the crystal; a device described in reference 17 was used rather then the shock tube because of its convenience and reliability. The device makes use of a rotating valve to switch a small test chamber between two large tanks of air at 
different pressure levels. It was concluded that the distortion due to charge leakage and temperature sensitivity could be negiected.

A convenient method of synchronizing the pressure trace on the oscilloscope with the interferometer photographs is to project both on the same film as in figure $2(b)$. With the oscliloscope set so as to suppress the time (horizontal) axis and with the film stationary, the pressure trace is focused on one-half the film and set above a convenient reference line in the interferogram, the wire which connects the. trailing edges of the blades. With the film in motion and the time axis thus provided, synchronization is complete. The amplitude of the pressure fluctuation at the measuring point in an interferogram is given by the intersection of the wire and trace. Since the crystal does not record a steady pressure, this fluctuating component of pressure must be added to the time mean static pressure measured with a wall static pressure tap directly opposite the crystal location (a 1/8-inch-diameter hole drilled in the opposite optical flat). The scale in figure 2(b) indicates the value of the mean pressure and the magnitude of the fluctuating component at the measuring point.

For pressure variations small with respect to the mean pressure, (in the present case $0.9 / 18$ ), the maximum error incurred in assuming the pressure at the measuring point to be equal to the mean pressure is small ( 5 percent). Of course, it was necessary first to measure these fluctuations to determine whether they could be ignored. Pressure distributions other than those for figure $2(b)$ were calculated neglecting the pressure fluctuations at the measuring point.

Measured pressure distributions: The most common ordinate in use in the presentation of pressure distributions from steady flows in cascades seems to be $S \equiv \frac{p_{0}-p_{\text {local }}}{\frac{1}{2} \mathrm{pW}_{1}{ }^{2}}$. That this is also a meaningful parameter for use in unsteady flows may be shown as follows:

In an unsteady flow $\dot{\psi}+p+\frac{\rho v^{2}}{2}=$ Constant $=\left(p_{0}\right)_{-\infty}$ where $\psi \equiv$ Velocity potential. If one integrates with respect to time over one cycle and requires that $\dot{\psi}$ make no net contribution for this period (otherwise the time mean value of $\left(p+\frac{1}{2} p v^{2}\right)$ monotonically increases or decreases.),

$$
\int p d t+\int \frac{1}{2} \rho v^{2} d t \equiv C T=\left(p_{0}\right)_{-\infty}{ }^{\top}
$$




$$
\int \frac{\left(p+\frac{1}{2} \rho v^{2}\right) d t}{\tau}=C=\int \frac{p_{0} d t}{\tau}=\text { Time mean } p_{0}=\left(p_{0}\right)_{-\infty}
$$

Therefore, for an unsteady flow,

$$
S \equiv \frac{\left(p_{0}\right)_{-\infty}-p}{\frac{1}{2} \rho W_{1}^{2}}=\frac{\dot{\psi}+\frac{1}{2} \rho v^{2}}{\frac{1}{2} \rho W_{1}^{2}}
$$

Although in a steady flow $S$ must always be positive, the preceding discussion shows that in an unsteady flow $S$ may be negative in certain regions and periods in which $\dot{\psi}$ is a large negative quantity.

The pressure distributions corresponding to each of the ten frames of figure $2(\mathrm{~b})$ for one period $T$ of the motion are shown in figure 30 . Generally, they bear little relation in form to the conventional graphs of pressure distribution. This is to be expected, however, for not only is the flow unsteady, but the effect of vortices in the field represents a major departure from conventional flow. Figures $30(\mathrm{c}), 30(\mathrm{~h})$, and $30(1)$ show negative values of $\mathrm{s}$. The circled data points in figure 30 are obtained from the interferogram fringes of figure $2(b)$ and by interpolating half fringes.

At the beginning of the cycle (frame I of fig. 2(b)), the blade has shed a counterclockwise vortex from its trailing edge, and the vortex shed from the leading edge of the preceding blade is coming into view. The latter vortex should have a strong effect upon the blade circulation according to the analysis presented previously. One would expect from this analysis that, in order for the Kutta condition to be maintained, the circulation around the blade in frame 3 , figure $2(b)$, would be large. This is verified qualitatively from the pressure distribution of figure $30(c)$ and from figure 31 , a graph of $c_{n}$ against $t / \tau$ as determined from the pressure distributions of figure 30 . On the other hand, the blade circulation decreases as a shed vortex leaves its leading edge and the effect on the blade pressure distribution is clearly indicated in figure 30 .

Local incidence angles at the blade were estimated from the schlieren pictures of figure 9, which show the wakes of the upstream nozzles darkened somewhat for reproduction purposes. These estimated values of $i$ are plotted in figure 32 against $t / \tau$. The error caused by the motion of the wakes and the difference in clearance between blade rows in figures 2 and 9 is neglected. Using a common time axis $t / \tau$, one may plot $C_{L}$, as calculated from figure 31 , against $i$ from figure 32 to obtain information about time lags between local incidence and $C_{L}$. Such a 
plot is shown in figure 33 which indicates that a phase lag does exlst between $i$ and $C_{L}$. This is the usual result, and the explanation seems to be that the presence of a counterclockwise vortex near the trailing edge has a negative effect on the blade circulation compared with its effect after having been washed dowñstream; the reverse is true for the leading-edge vortex as shown previously.

The pressure trace in figure $2(b)$ clearly shows the presence of the shed vortex. At the beginning of the cycle, when vortices are oriented so as to cause low velocity at the measuring point, the trace indicates high static pressure. At about the fifth frame, when a vortex is quite close to the pressure pickup and oriented so that the velocity is high there, the trace indicates low static pressure.

The magnitude of the blade force calculated from the sequence of figure 30 varied from 3 to 7.5 pounds. Although the blade force in the chord direction was calculated, these forces were small enough to be neglected. Hence the normal force is approximately equal to the blade vector force.

With the above experimental information, one can also show the "Böen effect," which is that in the unsteady growth of lift on a wing accompanying a sudden increase in the angle of attack an increase in the value of maximum lift occurs. In reference 13 a graph of lift coefficient against. incidence is given and the results may be compared with those of figure 33. At an incidence angle of $20^{\circ}$ (for which the steady state $C_{I}$ is a maximum of 0.68 from ref. 13), figure 33 gives values of 0.71 and 1.45 for $C_{I}$ during the unsteady flow. The maximum value of $C_{L}$ from figure 33 is 2.1. There is some error involved in this comparison since both $C_{L}$ and $C_{N}$ are based on the average upstream dynamic head rather than on the instantaneous local value; however, the above calculations appear to demonstrate that there is considerable departure from their quasi-steady stall characteristics by the airfoils in question during stall propagation as speculated previously.

In order to verify the design technique used to simulate a linear cascade with the circular cascade, the airfoll pressure distribution was calculated for $\beta=46.4^{\circ}$ from figure 27. The pressure distribution for the equivalent linear cascade (NACA 65(8)10) is given, for certain combinations of solidity, incidence, and other factors in reference 13. To compare the two distributions $1 t$ is only necessary to reduce the circular cascade distribution to that of a linear cascade, using the transformation given in reference 12. Of course, the comparison must be made for equal values of $\sigma, \lambda, 1$, and so forth. Thus, in $\mathrm{flg}-$ ure 34 , the transformed pressure distribution corresponding to $\beta_{1}=46.4^{\circ}$ has been compared with the appropriate distribution of reference 13 . 
This comparison indicates some success in the attempt in designing the circular cascade to duplicate the pressure gradients on the suction side of the blades. The apparent difference in blade loading is to be expected from the circular transformation.

Estimation of strength of shed vortices: It is possible to estimate the strength of a vortex moving past a point where the pressure is measured as in figure 2(b). Advantages of this method are that no measurement is required near the core of the vortex where losses are high and that the strength of an "equivalent potential vortex" can be estimated.

The variation of $p$, static pressure at the origin, when an isolated vortex $\Gamma$ moves along a line ( $y=$ Constant) with the free-stream velocity $C$ in an infinite field, can be determined in a coordinate system fixed to the vortex and is given by

$$
\text { - } \frac{p_{\infty}-p}{\frac{1}{2} p c^{2}}=\frac{\left(\frac{\Gamma}{2 \pi b c}\right)^{2}}{\frac{x^{2}}{b^{2}}+1}
$$

where the coordinates of the vortex are $(x, y=b)$.

An estimate of the circulation about an airfoil can be made by using the Kutta relationship $\mathrm{L}=\mathrm{pW} \Gamma_{0}$ and substituting approximate measured values of $I, \rho$, and $W$. Applying these two relationships to the ninth frame of figure $2(b)$, in which the blade circulation is nearly minimum (and neglecting the effect of adjacent blades), gives an estimated ratio of blade circulation $\Gamma_{0}$ to shed vortex strength $\Gamma$ of 0.19. However, because of the inaccuracies involved in this calculation, a direct examination of the interferometer pictures probably yields as good an answer. In figures 1 and 2 , it is observed that after the airfolls stalled there was virtually no difference in fluid density at points which are at the same chordwise position on the airfoll but on opposite sides. Neglecting the local time rate of change of velocity and losses, this indicates that the pressure and velocity were almost equal at these points and that the circulation about the airfoil must have been small. Therefore, it appears that the circular cascade airfoils shed nearly all of their bound vorticity when they stalled as assumed in the analysis.

Discussion of results. - The experimental results from the circular cascade indicate that stall propagation occurred for narrow bands of mean incidence during which the blades periodically shed nearly all their bound vorticity in the form of discrete vortices. It appears that the airfoils consistently stalled from their leading edges during 
stall propagation and that between stall cells the flow reattached to the suction side of the airfoils.

Although the washing of the guide-vane wakes over the cascade alrfoils had an influence on the flow as discussed previously, this does not appear to be the reason for the distinct bands of $\beta_{1}$ at which propagation occurred. The effect of Reynolds number on stall propagation was pronounced at some mean incldences but not at others. There was no pronounced effect on $C_{p}$ caused by the increase of clearance between blade rows for $\sigma$ of unity but $v_{p}$ increased and the number of cells decreased.

The analysis of reference 4 predicts that the effect of increased clearance is to decrease $V_{p}$ but that $V_{p}$ should increase for fewer cells. The present analysis indicates that the effect of decreasing the extension of the flow field downstream of the circular cascade, which was assoclated with the increase of clearance, should have caused a tendency for the number of cells to increase. The fact that the number actually decreased indicates that the effect of increased clearance was more important than the effect of decreased downstream field in determining the number of cells in this case.

It is noted that for configuration $B, V_{p} / C_{\theta_{1}}$ was essentially constant for each value of $\sigma$ but increased as $\sigma$ decreased. It is probable that this increase was caused in part by the effect of a decreased. number of boundary-layer time delays (assoclated with blade stalling). Furthermore, from figure 8 it can be seen that the induced effect of the circulation about airfoil (a) upon the shed vortex $\Gamma$ tends to decrease its transport velocity. This effect tends to increase $v_{p}$ as $\sigma$ decreases independently of the effect of boundary-layer time delay. The frequency of stall propagation appears to have approached the Kármán vortex frequency for an isolated plate as $\sigma$ decreased.

It was observed during the experimental work with the circular cascade that some of the stall-cell patterns were sensitive to small disturbances in the flow. For instance, in one case (for $\sigma=1$ ), stall propagation could be prevented by the insertion of a 1/8-inch-diameter hot-wire probe upstream of the cascade. On the other hand, at one time during the testing, 8 of the 54 cascade blades falled and were washed downstream and no pronounced effect was observed on stall propagation except that the flow was slightly less periodic than it was with uniform blade spacing. Therefore, the stabllity of the stall-cell patterns and the effect on stall propagation of destroying the axial symmetry of the flow appears to vary a great deal with mean flow condition in the circular cascade. No hysteresis or time-delay effect could be discerned between the stall-cell patterns in the circular cascade and the guidevane setting (incidence). 
Investigation in an Isolated Rotor

Description of apparatus and procedure.- This investigation was undertaken to extend the available experimental information about the detailed nature of flow through an isolated rotor during stall propagation. The equipment used was virtually the same as that described in reference 14, where a similar investigation was made for a guide-vane rotor and a guide-vane rotor-stator combination. Figure 35 is a schematic diagram of the research compressor showing the measuring stations.

The essential dimensions of the single-stage axial compressor used in this investigation are:

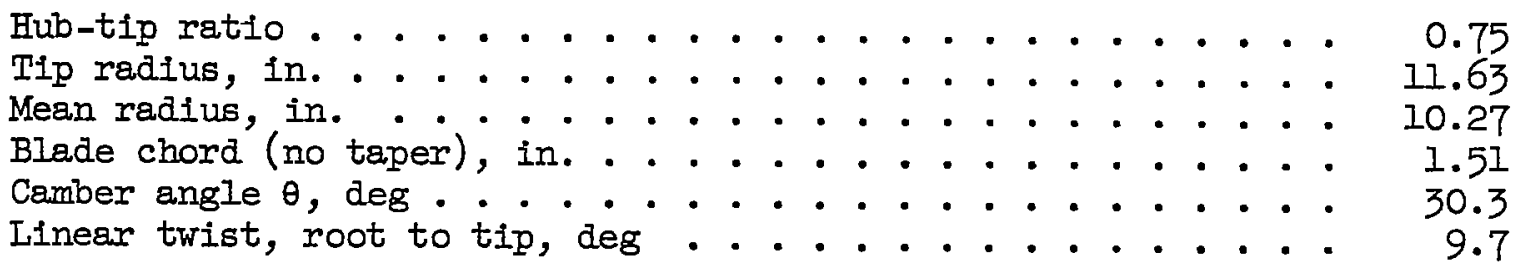

The blades were a circular-arc camber line, with an NACA 0010 thickness distribution (10 percent maximum thickness at 30 percent chord).

The rotor blades were unshrouded, and their bolt type of fastening allowed the stagger angle to be easily changed. The constant area annulus extended 29.8 inches upstream and 36.5 inches downstream of the rotor. Radial air-flow entrance was through screens. The outflow annulus was a diverging cone. The inner wall cone could be traversed, thereby varying the exit annulus area and throttling the flow.

The following three rotor configurations A, B, and C were tested:

\begin{tabular}{|c|c|c|}
\hline \multirow{2}{*}{ Configuration } & $\begin{array}{c}\text { Mean-radius stagger, } \\
\lambda, \text { deg }\end{array}$ & $\begin{array}{c}\text { Mean-radius solidity, } \\
\sigma\end{array}$ \\
\hline A & 30.2 & 1.02 \\
B & 30.2 & .51 \\
C. & 52.7 & 1.02 \\
\hline
\end{tabular}

Except where indicated, the rotor was operated at 1,500 rpm $(U=134 \mathrm{ft} / \mathrm{sec}$ at mean radius) for all configurations. 
The relative inlet velocity during stall propagation was 140 to $160 \mathrm{ft} / \mathrm{sec}$, which resulted in a Reynolds number based on the blade chord of 110,000 to 150,000 .

The conventional instrumentation consisted of inner- and outer-walI static pressure taps at five axial positions (C, E, F, FG, and $G$ in fig. 35), klel probes, a five-hole probe which was used for yaw measure. ments, and sphere-static probes. The pressure measurements were read on an inclined water mometer. The five-hole probe was nulled with the use of a Statham gage.

The probes used for measuring unsteady velocities were tungsten hot wires 0.00015 inch in dlameter and 0.044 inch long. The directcurrent circuits of two constant-current hot-wire anemometers were used. The voltage signals from the hot wires were applied directly to a Dumont 304 dual-beam oscilloscope. The amplifier and standard calibration procedure of the anemometer (model HWB, manufactured by Flow Corporation) for hlgh-frequency fluctuations was not usable, since large low-frequency fluctuations were being investigated. Drift in the direct-current circultry caused differences in the hot-wire callbrations immediately before and after a run of $1 / 2$ hour length as great as 25 percent. The calibration which came closest to being consistent with the steady-state readings before stall propagation started was used. It is felt that the accuracy obtained is sufficient to indicate certain trends in the data which will be discussed.

Since the hot-wire signal indicates only the component of velocity normal to it (over a wide range of angles within a small percentage correction), total velocity, axial component, or tangential component was measured by orienting the wire along radial, tangential, or axiai direction, respectively (assuming that the radial velocity component can be neglected).

An unsteady angle-measuring probe was developed during this investigation. As shown in figure 36, it consists of a tube which slips over a standard hot-wire probe. The tube is sealed at the end and has two small holes drilled $120^{\circ}$ apart at its midsection similar to a standard cylindrical yew probe. A thin wall is soldered inside the tube near the hot wire to reduce turbulence.

If the probe is not nulled in a streaming flow, there is flow through the probe which the hot wire sensès. This probe is sensitive to low velocities and it can be nulled within $\pm 2^{\circ}$. Its response is fast enough to indicate stall cells passing by it. Since the downstream velocity proflles during stall propagation were approximately square wave shapes, one could null the probe by observing the scope trace and thereby measure the angle of flow, either inside or outside the stall cells. 
To obtain permanent records of the oscilloscope traces, a Polaroid Corporation camera and a strip-film camera were used. The latter is a camera without a shutter but with a constant-speed film drive. The number of stall cells or $\phi$, the angle at which the cells extended downstream, was determined by the relative displacement of two traces, recorded on strip film, of hot-wire probes located at different axial stations.

The stall-cell frequency was determined by synchronizing a sinewave voltage from a frequency generator on the $x$-axis input with the hot-wire velocity signal on the $y$-exis input of the oscilloscope.

AlI probe readings except wall static pressures are values at the mean radius unless a radial traverse is indicated. Unsteady static pressure readings are at the outer wall and were measured with two inductance-type electrical pressure gages described in reference 18.

Experimenta.1 results - configuration A.- The time average pressure coefficient $C_{p}$ as determined by the average of the inner-and outerwall static pressures and the upstream relative head is plotted in figure 21 against $\beta_{c}$, where

$$
\begin{gathered}
c_{p_{C G}} \equiv \frac{p_{G}-p_{C}}{\frac{1}{2} \rho W_{C}{ }^{2}} \\
c_{p_{F H F}} \equiv \frac{p_{F}-p_{E}}{\frac{1}{2} \rho W_{C}^{2}}
\end{gathered}
$$

Pressures were measured at the stations indicated. The factors $W_{c}$ and $\beta_{c}$ were determined by measuring $C_{c}$ with a sphere static probe.

The number of stall cells and their velocity of propagation are given in figure 37, together with values of $v_{p}$ predicted in reference 4 , for no downstream pressure fluctuations by

$$
\frac{V_{p}}{C_{X}}=\frac{2\left(1-C_{p_{C G}}\right)}{\sin 2 \beta_{c}\left(\frac{L B \pi}{2 \cos \beta_{G}}+1\right)}
$$


where $B \equiv$ Number of cells. The values predicted in reference 2 are the same as in the "channel theory" of reference 3 for no phase lag,

$$
\frac{V_{p}}{C_{x}}=\frac{1}{\sin 2 \beta_{c}}
$$

In figure 38 are shown unsteady wall static gage pressures measured at stations $E$ and FG. It is observed that the upstream fluctuations are conslstently several times as large as those downstream.

A series of hot-wire traces corresponding to messurement of $C_{\theta_{G}}$ and $C_{x_{G}}$ is shown in figure 39. Traces for $C_{x_{G}}$ and $C_{x_{E}}$ are shown in figure 40. The apparent difference in the average values of $c_{x_{G}}$ and $C_{X_{E}}$ in figure 40 is probably due to error in calibration of the hot wires caused by "direct-current drift" in the hot-wire circuitry as discussed previously, since these values must be constant because of continuity.

In figure 41 are given the results of calculating $\left(W_{\theta_{G}}\right)_{0}$ and $\left(W_{\theta_{G}}\right)_{1}$ from the hot-wire traces of figure 39 . In figure 42 are $\left(C_{x_{G}}\right)_{0}$ and $\left(C_{x_{G}}\right)_{1}$ as calculated from figure 40 and $C_{x_{C}}$ from sphere static probe readings.

The measured values of $\left(C_{x_{G}}\right)_{0}$ are less than those of $C_{x_{C}}$ for $\beta_{c}<58^{\circ}$ in figure 42. This does not appear to be physically possible because of the blockage effect of the cells. It is felt that again this is a calibration discrepancy since $C_{x_{C}}$ must be the average value of $C_{X_{G}}$. because of continuity. Using this fact, the value of $\left(C_{X_{G}}\right)_{0}$ estimated for the hot-wire trace at $\beta_{c}=56.7^{\circ}$ is $95 \mathrm{ft} / \mathrm{sec}$. Since all the data indicate that $\left(C_{x_{G}}\right)_{0}$ is essentially constant with throttling except for extremely high values of $\beta_{c}$, it is probably a good approximation to take $\left(C_{x_{G}}\right)_{0}$ as constant and equal to $95 \mathrm{ft} / \mathrm{sec}$ as indicated in figure 42.

The flow angle $\left(\alpha_{G}\right)_{0}$ as measured by the hot-wire angle probe, $\beta_{G}$ as determined by a standard five-hole yaw probe, and $\left(\beta_{G}\right)_{0}$ are 
plotted against $\beta_{C}$ in figure 43. The flow angle in the coordinate system fixed to the rotor $\left(\beta_{G}\right)_{0}$ was calculated from an average value of $\left(\alpha_{\mathrm{G}}\right)_{0}, 40^{\circ}$, and $\left(c_{\mathrm{X}_{\mathrm{G}}}\right)_{0}$ taken as $95 \mathrm{ft} / \mathrm{sec}$.

From figure 41 it is determined that $\left(W_{\theta_{G}}\right)_{1}$ was greater by 4 to 18 percent than $\left(W_{\theta_{G}}\right)_{0}$ (as $\beta_{C}$ increases). This result correlates with the experimental observation that the stall-cell angle $\varnothing$ was negative and smaller than $-15^{\circ}$ for all the stall-cell patterns of configuration A.

Experimental results - configuration B. - To determine the effect of blade spacing, half the blades of configuration A were removed resulting in configuration B. The number of cells and their propagation velocity are shown in figures 44 and 45 which indicate that periodic one- and twocell patterns existed for only a narrow range of high incidences for configuration $B$.

Experimental results - configuration C. - To ascertain the effect of blade stagger angle, the stagger was increased $22 \frac{1}{2}^{\circ}$ from configuration $\mathrm{A}$ to configuration $C$ and comparative data were taken. The measured results are indicated in the following figures:

\begin{tabular}{|c|c|c|}
\hline Figure & Results plotted against $\beta_{C}$ & $\begin{array}{l}\text { Corresponding } \\
\text { figure for } \\
\text { configuretion A }\end{array}$ \\
\hline $\begin{array}{l}22 \\
46 \\
47 \\
48\end{array}$ & $\begin{array}{l}C_{p_{C G}} \text { and } C_{p_{E F}} \\
\text { Number of cells, } V_{p} \text {, and predicted } V_{p} \\
\text { Hot-wire traces of } C_{x_{E}} \text { and } C_{x_{G}} \\
\text { (1) } C_{x_{C}} \text { measured by sphere static probe } \\
\text { (2) }\left(C_{x_{G}}\right)_{0} \text { and }\left(C_{x_{G}}\right)_{i} \text { as calculated from } \\
\text { figure } 47 \\
\text { (3) }\left(C_{x_{G}}\right)_{0}=76 \mathrm{ft} / \text { sec as calculated from } \\
\text { figure } 49 \\
\text { (4) } \beta_{G} \text { as determined with standard five-hole } \\
\text { yaw probe } \\
\text { (5) }\left(\alpha_{G}\right)_{0} \text { as measured with hot-wire angle } \\
\text { probe } \\
\text { (6) }\left(\beta_{G}\right)_{0}=45.30 \text { calculated taking average } \\
\left(\alpha_{G}\right)_{0}=37.5^{\circ} \text { and average } \\
\left(C_{x_{G}}\right)_{0}=76 \mathrm{ft} / \mathrm{sec}\end{array}$ & $\begin{array}{c}21 \\
37 \\
40 \\
42,43\end{array}$ \\
\hline
\end{tabular}


Figure 49 shows a strip-film record of a hot-wire trace of axial velocity measured at station $G$ while the throttle was continuously opened so that the compressor operating condition changed from two, to one, to zero cells. For $\left(\mathrm{C}_{\mathrm{x}_{G}}\right)_{0}$, the trace $\mathrm{Is}_{\mathrm{s}}$ at the top edge of the film for the entire strip. During this strip film, which took about 5 seconds, direct-current anplifier drift was negligible. (The calibration was checked immediately after the run, and it had not changed during the run.) The value of $\left(C_{x_{G}}\right)_{0}$ is observed to be almost constant and is calculated to be $76 \mathrm{ft} / \mathrm{sec}$, which compares well with the average of the values from the hot-wire traces of figure 47.

For configuration $C$, the angle $\phi$ between the stall cells and rotor axis was determined from the measurement of phase lag between two traces of hot wires inserted at stations $G$ and $K$. It was determined that for the one-cell pattern $\phi=19^{\circ}$ and for the two-cell pattern $\phi=16^{\circ}$. The error of measurement probably was less than 10 percent.

Comments on compressor operation. - Just before rotating stall started, as the throttle was closed, a "mushy" flow, as it has been called, was observed near the hub and tip as indicated by a "hashy" hotwire trace. A very weak rotating stall might have been present at the hub for configuration $C$. The relative magnitudes of the mush at 1/4 inch from the hub and rotating stall of one cell can be seen from hot-wire traces of figure 50 for configuration $C$. The mush was less than one-fifth the amplitude of rotating stall and was not investigated further. Rotating stall measurements were taken through the operating range until surge started. Although unperiodic propagation could atill be observed along with the surge (since the surge was of much lower frequency), no attempt was made to take data and separate the two effects. The variation of velocity fluctuation with axial distance from the rotor was measured at one operating condition for configuration $A$ as is shown in figure 51. It is noted that the amplitude of the velocity fluctuations decreases rapidly with distance upstream but that the fluctuations persist much farther downstream. At station $\mathrm{K}, 26$ inches downstream, the amplitude of the fluctuations is about one-half that at station $G$.

Upon throttling the flow, rotating stall always started as two cells then, as the flow was increased, the two cells changed to one. However, if the axial symmetry of the flow was destroyed by placing an obstacle (such as the observer's hand) in the inlet or outlet of the compressor, the one-cell pattern could be forced to appear before the two-cell pattern as the flow was throttled.

If the compressor were operated at a value of $\beta_{C}$. where the number of stall cells changed, the cell pattern sometimes shifted back and forth between the two numbers of cells. 
Radial traverses of the hot-wire probe during stall propagation indicated that the flow was fairly two-dimensional for all the observed stall-cell patterns, although in some cases an estimated 25 percent smaller fluctuation in velocity was observed at the hub.

To determine the effect of Reynolds number on rotating stall characteristics, the rotor speed was held at $500,750,1,000,2,000$, and $2,500 \mathrm{rpm}$ and the flow was throttled. A comparison of the number of cells and propagation velocity at three speeds is shown in figures 52 and 53 as functions of $\beta_{C}$. "Constant geometry" runs were made for two constant throttle and rotor settings. The results of these runs and the previous one are shown in figure 54 , which is a plot of the number of cells and $\beta_{C}$ as a function of $R_{C}$. As indicated in these figures, there is a hysteresis effect between the number of cells for some values of $R e_{C}$ and $\beta_{C}$. The data points at minimum values of $\beta_{C}$ indicate the onset of stall propagation and the points at maximum values of $\beta_{C}$ indicate the end of periodic stall propagation. (For higher values of $\beta_{C}$ high-frequency velocity disturbances and surge existed.) The data plotted in figures 52, 53, and 54 indicate that there was not much effect of Reynolds number above 100,000 or $1,500 \mathrm{rpm}$ but that for lower values the range of $\beta_{C}$ for which periodic propagation existed is shifted up. As Reynolds number was lowered, the one-cell and then the two-cell patterns disappeared, but there was not much effect upon propagation velocity. It was found that there was virtually no difference in $C_{p_{C G}}$ at corresponding values of $\beta_{C}$ for 500 and $1,500 \mathrm{rpm}$.

Axial velocity profiles as determined from measurements made with a standard five-hole yaw probe for configuration $C$ are shown in figure 55. They indicate that there is some error involved in assuming mean radius measurements to be representative but that this error is less during than before the onset of stall propagation. The difference in area under the profiles measured during stall propagation indicates the error inherent in using a standard five-hole probe in an unsteady flow.

Discussion of experimental results. - The hot-wire measurements of figures 40 and 47 indicate that the downstream axial velocity profile can be approximated with a square wave which changes with throttling only along the abscissa. That is, $\left(C_{x_{G}}\right)_{0}$ and $\left(C_{x_{G}}\right)_{i}$ in figures 42 and 48 remain essentially constant with $\beta_{C}$ and maintain approximately a 4:1 ratio, but the number of cells and the width of the cells change so that their blockage effect increases contimuously with throttling. The data appear to correlate with those from reference 19 in indicating that during stall propagation the velocity triangles outside the stall 
cells were nearly the same as those for $c_{p_{\max }}$ (before the onset of stall propagation).

The unsteady pressure measurements of figure 38 indicate that the fluctuations were several times greater I inch upstream of the rotor than they were 5.6 inches downstream. (When a pressure gage was inserted 7 inches downstream at station $G$, virtually no fluctuations could be observed in the trace.) As a stall cell approached the upstream gage, the static pressure at this point rose to a value greater than the absolute total head far upstream but less than the total head relative to a coordinate system fixed to the stall-cell pattern. The static pressure then dropped as the stall cell passed by the gage. There was a pressure drop through the rotor in the stall cells. This qualitative description of the pressure field is predictable from the flow model used in the analysis from a consideration of the velocities induced by the stall cell in a coordinate system fixed to the stall-cell pattern.

The hot-wire traces for $C_{x_{\mathrm{E}}}$ in figures 40 and 47 indicate that, as $\beta_{C}$ increased, the cells grew wider and that the axial velocity profile just upstream of the rotor changed shape as they grew wider. The "sag" in the profile caused by the blockage of the stall cell changed from a spike form for a thin cell to a double spike indicating that there was more flow through the center of the wide cell than near its edges. Since the trace did not ever go to zero (the minimum value being $25 \mathrm{ft} / \mathrm{sec}$ ), in no case was there flow reversal at station $E$ during stall propagation. This fact was verified independently of the calibration of figures 40 and 47 by quickly pulling the hot-wire probe at station $E$ out of the compressor and observing that for zero air velocity the trace went considerably lower than the minimum displacement for the double-spike wave form during stall propagation. A check was also made by quickly turning the wire $90^{\circ}$ from a tangential to axial direction and observing the same result as above for zero velocity normal to the wire. The number of cells is observed to increase after the double-splke form appeared, which suggests that the double-spike form split into two cells, a tendency predicted by the preceding analysis. From the strip-film trace of figure 49, it is observed that the opposite sequence occurred when the flow was increased through the rotor muning at constant revolutions per minute. At first there were two stall cells $180^{\circ}$ apart, then the cells gradually moved together forming a double-spike pattern and then a single -spike pattern.

The hot-wire traces of figures 40,47, and 49 show that the shape of the stall-cell pattern is not perfectly constant in time or axisymmetric (as assumed in the analysis for a given mean flow and even number of cells). This fact appears to explain why one cell of a two-cell pattern might split and thus form a three-cell pattern as the flow is 
throttled instead of both cells splitting simultaneously. However, the trend for the observed shapes of the stall-cell pattern with throtting appears to correspond qualitatively with the trend predicted by the analysis.

$$
\text { In figures } 44 \text { and } 45 \frac{V_{p}}{C_{x_{C}}} \text { and } \frac{V_{Q}}{U} \text { as functions of } \beta_{C} \text { are com- }
$$

pared for configurations $A, B$, and $C$ and for data from reference 19. It is noted that a.1I stall-cell patterns observed herein revolved at $0.48 \mathrm{U}<\mathrm{v}_{\mathrm{p}}<0.59 \mathrm{U}$, or approximately half wheel speed. In figure 44, $V_{p}$ increased slightly when the solidity of the cascade was reduced in agreement with the trend observed in the circular cascade.

Not much effect on propagation velocity occurred with the change of stagger angle or with Reynolds number change above 100,000. However, the number of cells was greatly affected by the change of solidity, of stagger, and of Reynolds number below 100,000.

The propagation velocities predicted by the small perturbation theories of references 2, 3 (no phase lag), and 4 as shown in figures 37 and 46 appear to overestimate the experimental values by as much as 50 percent. The correlation of the data with the theory of reference 4 appears to be well described by the correlation given on page 24 of that reference "the values of propagation velocity predicted when the boundarylayer deley is neglected should be larger than those obtained experimentally, with the difference most pronounced for the case of disturbances covering only a few airfoils when the boundary-layer delay will have an important effect. An increase in the wave length of the stall cells should be accompanied by an increase in propagation velocity, if other variables are unchanged."

The effect of number of cells on propagation velocity as predicted above appears to be borne out quite conclusively by the data plotted in figures 37 and 46.

In figure 56 the function

$$
A=\text { Discharge coeficient } \equiv \frac{\cos \beta_{C}}{\cos \beta_{G}\left(1-C_{p_{C G}}\right)^{1 / 2}}
$$

is plotted against $\beta_{C}$. The values of $\beta_{G}$ were those determined with the standard yaw probe. In references 5 and 4 it was predicted that small perturbation stall cells may form when the slope of this curve passes through the origin. There seems to be fair agreement from figure 56 with this prediction. 
It is observed in figures 21 and 22 that the slopes of the curves of $C_{p}$ against $\beta_{C}$ for configurations $A$ and $C$ both approached zero when stall propagation occurred.

In reference 19 tests were made with a rotor of solidity $=1$, $28.5^{\circ}$ stagger, NACA $65(12) 10$ airfolls, and hub-tip ratio of 0.9 . As shown in figure 45 , there is overall qualitative agreement between the data for configuration $A$ and those in reference 19. Stall propagation started at nearly the same incidence and $V_{Q} / U$ was nearly the same, but there were differences in the number of cells and the measured pressure fluctuations. In reference 19 it is reported that the pressure fluctuations downstream were about 60 percent of those upstream. In the present investigation, they are found to be three to five times as great upstream as they are downstream; however, the gages are closer together then they are in reference 19, and the cells extend more uniformly from root to tip than the two- and three-cell petterns of reference 19.

Correlation of Analytical and Experimental Results

Verfication of the assumptions made in the analysis appears from the following experimental results:

(1) From figures 1, 2, 3, 12, and 23, it is observed that during stall propagation the blades in the circular cascade shed vortices upon entering or leaving stall cells and lose nearly all their bound vorticity when they enter the cells.

(2) From the approximate square weve shape of the velocity profiles downstream of the rotor during stall propagation, as indicated by the hot-wire traces of figures 40 and 47 , It appears that the vortex flow model may be used to represent the actual fliow through the rotor. Furthermore, the square wave shape of the downstream velocity profiles tends to support the idea that the rotor blades stalled from their leading edges as the blades in the circular cascade are observed to have done. In reference 11 , page 66 , it is shown that the rate at which vorticity. is shed from an airfoil is $W^{2} / 2$ where $W$ is the free-stream velocity at the separation point of the boundary layer. Since immediately downstream of the rotor blades the vorticity is observed to have been concentrated along the stall-cell boundaries, - it might be argued that, in order for the blades to have shed vorticity quickly enough to have established this pattern, the point at which the boundary layer separated must have been located where $W$ was large, near the leading edge. The validity of this speculation is not essential, however, since the present analysis is independent of the location of separation. 
(3) The observation from the section "Investigation in an Isolated Rotor" that the ratio of downstream axial velocity outside the cell is about four times as great as that inside indicates that the approximation of $N=P=I$ in the analysis may be a reasonable one in view of the discussion in the section "Derivation of Expressions for Propagation Velocity" which indicates that this approximation is not critical to the resulting prediction for $V_{p}$.

In the present analysis, an approximation is made for the flow far downstream of a continuous blade row during stall propagation. The results of the analysis indicate why some observers have found incongruous experimental results from linear cascades of finite length. It appears that, as was first described in reference 3, the blade stallunstall characteristics depend strongly on the nature of the unsteady flow induced upon them by the downstream flow pattern. In a Iinear cascade of finite length, the downstream flow pattern is quite different from that for a continuous circular cascade or a rotor. Even though there may be many blades in the linear cascade, a fully developed, translating, downstream flow pattern as described in the analysis is precluded by the end wall effects. Therefore, the present analysis is valid only for an infinitely long linear cascade, or an isolated rotor, where the downstream flow field extends far from the cascade.

The tendency for the stall cell to split into two as its width increases, as predicted by the analytical flow model, appears to be supported by the measured axial velocity profiles just upstream of the rotor given in figures 40,47 , and 49 . Furthermore, this tendency might explain why smaller stall cells (greater numbers of cells) existed in the circular cascade than in the rotor. Since the two-dimensional-flow field of the circular cascade extended only a few blade chords downstream of the cascade, the discussion in the section "Prediction of a Trend for the Number of Cells" indicates that the cells should be thinner (smaller $n$ values in fig. 10) than they are for the rotor. This fact, together with the assumption that there must be enough total blockage from the cells to cause $\left(W_{2}\right)_{0}$ to be nearly the same as it is for $C_{p_{\max }}$ (as observed for the rotor), implies that there should be a greater number of cells in the circular cascade than in the rotor.

Equation (12) shows that, for the vortex flow model assumed in the analysis, stall cells can exist only for $c_{p}>\cos ^{2} \beta_{1}$. In figure 57 this minimum value of $c_{p}$ is plotted against $\beta_{1}$. Measured values of $C_{p}$ are plotted for increasing values of $\beta_{1}$ until the onset of stall propagation. Propagation started after $C_{p}>\cos ^{2} \beta_{I}$ in the three rotors tested. (This was not always true for the circular cascade.) 
Equation (II) shows that for the assumed type of propagation to exist $W_{\theta_{1}}>W_{2}$. If it is assumed that $C_{x_{1}}=C_{x_{2}}$ and $\beta_{2}=\lambda$, this condition becomes $\cot \beta_{1}<\cos \lambda$, giving a minimum value of $\beta_{1}$ for which stall propagation can exist in a cascade of stagger $\lambda$. This minimum value of $\beta_{1}$ and the corresponding measured values of $\beta_{1}$ where stall propagation started in the test rigs are plotted in figure 58. Also plotted is $\cot \beta_{1}=0.866 \cos \lambda$ (which gives $v_{p}=\frac{W_{\theta_{I}}}{2}$ from eq. (11) if $C_{x_{1}}=C_{x_{2}}$ and $\beta_{2}=\lambda$ ). It appears that this latter curve correlates rather well with the values of $\beta_{1}$ where stall propagation actually began in the test rigs.

Predicted values of propagation velocity for the vortex flow model are given by equations (11) and (12). Correlation of the rotor data with equation (12) is shown in figures 37 and 46 where $C_{p_{C G}}$ from figures 21 and 22 wes used. Correlation of the data from reference 19 with equation (12) is shown in table $I$ where $C_{p_{3-7}}$ from that reference was used. It appears that the predicted values of $v_{p}$ from equation (12) are considerably greater than the measured values. It is felt that the reason for this trend Iles in the fact that $p_{2}-p_{1}$ was assumed to be given by isentropic flow outside stall cells in developing equation (12). The presence of blade wakes in the actual flow outside the stall cells causes $\left(\beta_{2}\right)$ o to be less for a given measured value of $\Delta p$ than it is in the assumed ideal flow. Therefore, for a measured value of $\Delta p$, the value of $V_{p}$ should be less than that predicted by equation (12).

Using the average measured values for $\left(\beta_{\mathrm{G}}\right)_{0}$ and $\left(C_{\mathrm{x}_{\mathrm{G}}}\right)_{0}$ indicated in figures 42,43 , and $48, \mathrm{~V}_{Q} / \mathrm{U}$ calculated from equation (1I) is 0.57 for configuration $A$ and 0.60 for configuration $B$. The correlation with the measured values of $\mathrm{V}_{Q} / \mathrm{U}$ as shown in figure 45 appears to be good considering the accuracy of measurement, the departure of the flow from two-dimensionality, and the simplifying assumptions in the analysis. Equation (II) appears to predict propagation velocities more closely than equation (12), probably because it is less sensitive to the effect of blade wakes outside stall cells.

In table $I$ is shown the correlation of data from reference 19 with equation (II) using $\mathrm{C}_{\mathrm{p}_{3-7}}$ from reference 19 and assuming that

$$
\left(\beta_{G}\right)_{0}=\text { Constant }=28.5^{\circ}=\lambda
$$




$$
\mathrm{c}_{\mathrm{x}_{1}}=\left(\mathrm{c}_{\mathrm{x}_{2}}\right)_{0}
$$

for the two- and three-cell patterns and that $c_{x_{1}}=0.8\left(c_{x_{2}}\right)_{0}$ for the one-cell pattern, which are estimates based upon the data presented in reference 19. In table II 1s shown the correlation of data from the circular cascade with equation (12). It is observed that equation (12) overestimates the measured values of $\frac{V_{p}}{C_{X_{I}}}$ considerably for the circular cascade. However, good correlation cannot be expected since the assumption in the analysis that the flow fleld extends infinitely far downstream of the cascade is not a valid approximation for the circular cascade. Furthermore, the analysis is not valid for a radial flow field.

In table III is shown the correlation with equation (12) of data from reference 14 for a guide-vane rotor stage relative to the rotor.

\section{CONCLUDING REMARKS}

Using approximations suggested by visual observation of the flow through a circular cascade, a vortex flow model of stall propagation in an isolated blade row has been developed. The equation derived from analysis of the vortex flow model (eq. (II)) appears to predict the propagation velocities measured in an isolated rotor within 15 percent. Stallcell configurations observed in an isolated rotor were found to be nearly two-dimensional and to propagate at approximately half wheel speed. The stall cells consisted of regions where the axial velocity was small and extended downstream from the rotor in a direction parallel to the rotor axis within $\pm 20^{\circ}$.

The number of cells increased as the flow through the rotor was throttled. A qualitative prediction of the analysis is that a stall cell of the type assumed should have a tendency to split into two cells as it grows in peripheral extent. This tendency may be the reason for the observed trend in the number of cells.

Massachusetts Institute of Technology, Cambridge, Mass., August 13, 1956. 


\section{REFERENCES}

1. Emmons, H. W., Pearson, C. E. and Grant, H. P.: Compressor Surge and Stall Propagation. Trans. A.S.M.E., May 1955, pp. 455-467.

2. Marble, Frank E.: Propagation of Stall in a Compressor Blade Row. Tech. Rep. No. 4 (Contract AF 18(600)-178), C.I.T., Daniel and Florence Guggenheim Jet Propulsion Center, Jan. 1954.

3. Sears, W. R.: A Theory of "Rotating Stall" in Axial-Flow Compressors. Cornell Univ,, Graduate School of Aero. Eng., Jan. 1953.

4. Stenning, Alan H., Kriebel, Anthony R., and Montgomery, Stephen R.: Stall Propagation in Axial-Flow Compressors. NACA TN 3580, 1956.

5. Anton, Leo: Formation of a Vortex at the Edge of a Plate. NACA TM 1398, 1956.

6. Fage, A., and Johansen, F. C.: On the Flow of Air Behind an Inclined Flat Plate of Infinite Span. R. \& M. No. 1104, British A.R.C., 1927 .

7. Milne-Thomson, L. M.: Theoretical Aerodynamics. D. Van Nostrand Co., Inc., or Macmilian and Co., Itd., 1948.

8. PrandtI, I., and Tietjens, O. G.: Fundamentals of Hydro- and Aeromechanics. McGraw-Hill Book Co., Inc., 1934.

9. Schlichting, H.: Berechnung der reibungslosen inkompressiblen Stromung für eln vorgebenes ebenes Schaufelgitter. VDI-Forschungsheft 447, Forsch. Geb. Ing.-Wes., Ausg. B., Bd. 21, 1955.

10. Lin, C. C.: On the Motion of Vortices in Two Dimensions. Appl. Math. Ser. No. 5, Univ. of Toronto Studies, Univ. of Toronto Press, 1943.

11. Fluid Motion Panel of the Aeronautical Research Committee and Others: Modern Developments in Fluid Dynamics. Vol. I, S. Goldstein, ed., The Clarendon Press (Oxford), 1952.

12. Kriebel, A. R., and Stenning, A. H.: A Cascade Tunnel for Invest1gation of Rotating StaIl. Rep. No. 26, Gas Turbine Lab., M.I.T., Aus. 1954.

13. Herrig, L. Joseph, Emery, James C., and Erwin, John R.: Systemat1c Two-Dimensional Cascade Tests of NACA 65-Series Compressor Blades at Low Speeds. NACA TN 3916, 1957. (Supersedes NACA BM I51G31.) 
$8 \mathrm{R}$

14. Montgomery, S. R., and Braun, J. J.: Investigation of Rotating Stall in a Single-stage Axial Compressor. NACA TN 3823, 1957.

15. DeFrate, I. A., Barry, F. W., and Bailey, D. Z.: A Portable MachZehnder Interferometer. Meteor Rep. No. 51, Gas Turbine Lab., M.I.T., Feb. 1950.

16. Draper, Charles S., McKsy, Walter, and Lees, Sidney: Instrument Engineering. Vol. III, pt. I, McGraw-Hill Book Co., Inc., 1953.

17. Livengood, James C.: Improvement of Accuracy of Balanced-Pressure Indicators and Development of an Indicator Calibrating Machine. NACA TN $1896,1949$.

18. Patterson, John I.: A Miniature Electrical Pressure Gage Utilizing a Stretched Flat Diaphragm. NACA TN 2659, 1952.

19. Costilow, Eleanor I., and Huppert, Merle C.: Rotating-Stall Characteristics of a Rotor With High Hub-Tip Radius Ratio. NACA TN 3518, 1955. 
TABLE I

CORRFIATION OF DATA FROM REFERENCE 19

WITH EQUATIONS (1I) and (12)

\begin{tabular}{|c|c|c|c|c|c|}
\hline $\begin{array}{c}\beta_{1}, \\
\text { deg }\end{array}$ & $C_{p_{3-7}}$ & $\begin{array}{c}\text { Number of } \\
\text { cells }\end{array}$ & $\left(\frac{V_{p}}{C_{x_{1}}}\right)_{\text {measured }}$ & $\left(\frac{v_{p}}{C_{x_{1}}}\right)_{\text {eq. (12) }}$ & $\left(\frac{V_{p}}{C_{x_{1}}}\right)_{\text {eq. (11) }}$ \\
\hline 55 & 0.46 & 2 & 0.53 & 0.80 & 0.58 \\
56 & .43 & 3 & .48 & .87 & .54 \\
58 & .33 & 1 & .89 & 1.19 & .87 \\
\hline
\end{tabular}


TABLE II

CORRELATION OF DATA FROM CIRCULAR CASCADE WIIH EQUATION (12)

(a) Data from reference 4; $\sigma=1$

\begin{tabular}{|c|c|c|c|c|}
\hline $\begin{array}{l}\beta_{1}, \\
\text { deg }\end{array}$ & $\mathrm{c}_{\mathrm{p}}$ & $\begin{array}{l}\text { Number of } \\
\text { cells }\end{array}$ & $\left(\frac{V_{p}}{C_{r_{1}}}\right)_{\text {measured }}$ & $\left(\frac{V_{p}}{C_{x_{I}}}\right)_{\text {eq. }}(-12)$ \\
\hline $\begin{array}{l}63 \\
64 \\
66 \\
68\end{array}$ & $\begin{array}{l}0.48 \\
0.46 \\
0.41 \\
.36\end{array}$ & $\begin{array}{c}9 \\
9 \\
10 \text { to } 12 \\
12\end{array}$ & $\begin{array}{r}0.60 \\
.64 \\
.70 \\
.70\end{array}$ & $\begin{array}{r}0.80 \\
.86 \\
1.03 \\
1.22\end{array}$ \\
\hline
\end{tabular}

$a_{\text {Extrapolated from data. }}$

(b) Configuration A; $\sigma=1$

\begin{tabular}{|l|c|c|c|c|}
\hline $\begin{array}{l}\beta_{1}, \\
\text { deg }\end{array}$ & $C_{p}$ & $\begin{array}{c}\text { Number of } \\
\text { cells }\end{array}$ & $\left(\frac{V_{p}}{C_{r_{1}}}\right)_{\text {measured }}$ & $\left(\frac{V_{p}}{C_{x_{1}}}\right)_{\text {eq. }}$ (12) \\
\hline 55 & 0.33 & 15 to 20 & 0.24 & 1.4 \\
60 & .29 & 13 to 14 & .54 & 1.3 \\
68 & .25 & 16 & .75 & 1.6 \\
81 & .12 & 5 & 1.6 & 4.3 \\
\hline
\end{tabular}

(c) Configuration B; $\sigma=1$

\begin{tabular}{|c|c|c|c|c|}
\hline $\begin{array}{c}\beta_{I}, \\
\mathrm{deg}\end{array}$ & $C_{p}$ & $\begin{array}{c}\text { Number of } \\
\text { cells }\end{array}$ & $\left(\frac{V_{p}}{C_{r_{1}}}\right)_{\text {measured }}$ & $\left(\frac{V_{p}}{C_{x_{I}}}\right)_{\text {eq. (12) }}$ \\
\hline 58 & $\begin{array}{r}0.45 \\
.36\end{array}$ & $\begin{array}{c}9 \text { to } 10 \\
11\end{array}$ & $\begin{array}{c}0.55 \\
.83\end{array}$ & $\begin{array}{c}0.82 \\
1.6\end{array}$ \\
\hline
\end{tabular}


TABTE III

CORRETATION OF DATA FROM REFPERENCE 14

WITH EQUATION (12)

\begin{tabular}{|c|c|c|c|c|}
\hline$\beta_{1}$, & $C_{p}$ & $\begin{array}{c}\text { Number of } \\
\text { deg } 11 \text { s }\end{array}$ & $\left(\frac{V_{p}}{C_{x_{1}}}\right)_{\text {measured }}$ & $\left(\frac{V_{p}}{C_{x_{1}}}\right)_{\text {eq. (12) }}$ \\
\hline 67.5 & 0.30 & 8 & 0.9 & 1.4 \\
72.5 & .25 & 9 & 1.1 & 1.8 \\
75.0 & 8.23 & 1 & 2.3 & 2.2 \\
77.5 & 0.20 & 1 & 3.0 & 2.8 \\
80.0 & 0.16 & 3 & 3.0 & 3.6 \\
\hline
\end{tabular}

$a_{\text {Rxtrapolated from data. }}$ 


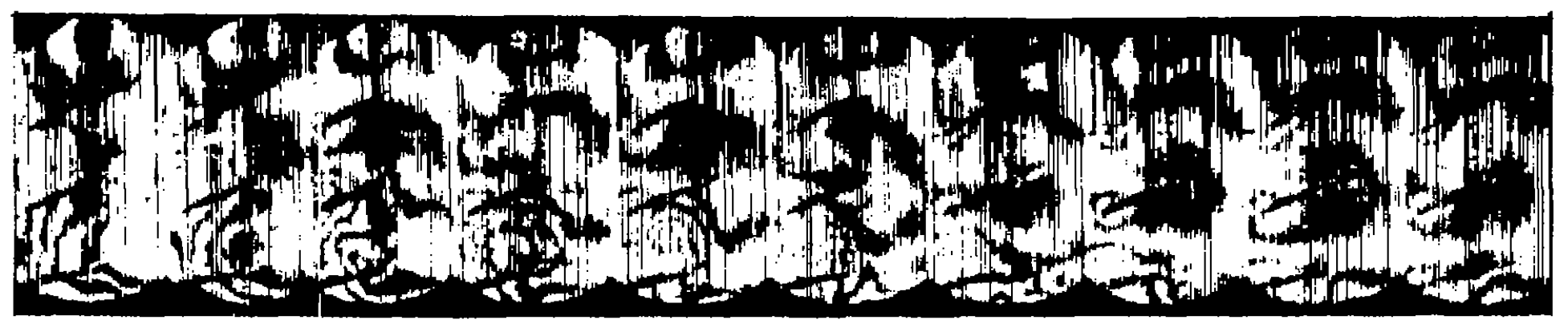

㕝
口
是
点
$\stackrel{5}{5}$

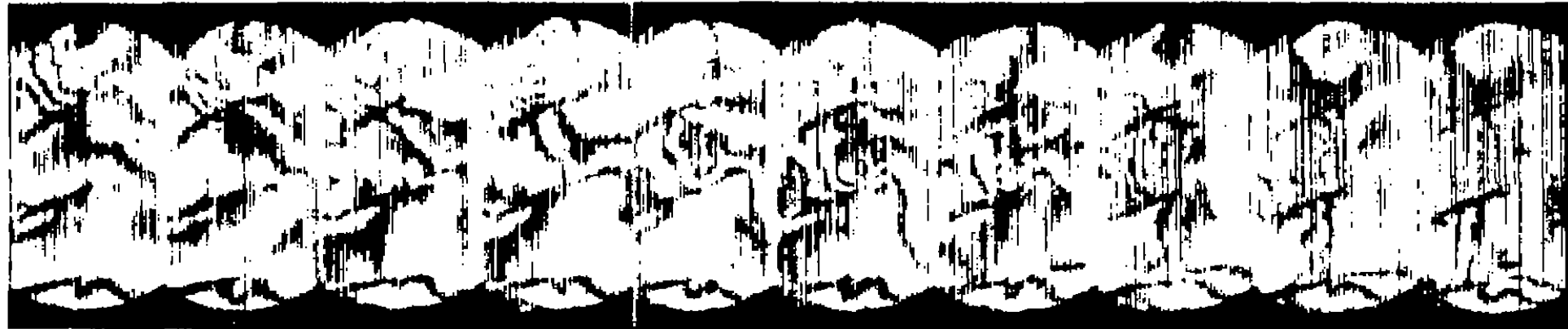

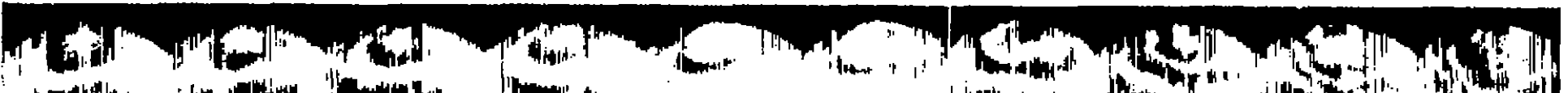

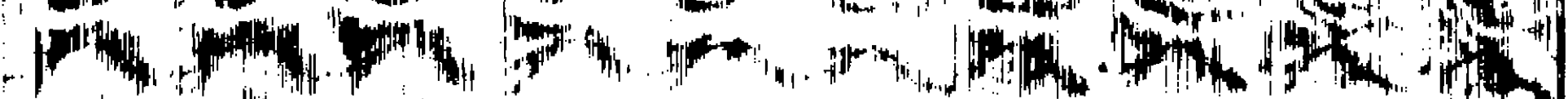

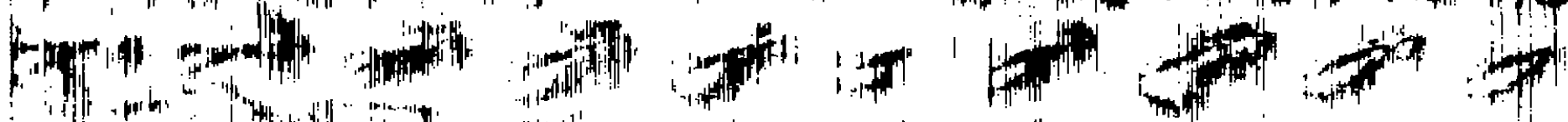
(n)

$$
\text { (a) } \beta_{1}=57^{\circ} \text {. }
$$

Figure 1.- Interferometer f1lms. Configuration $B ; \sigma=1$; time is from right to left. 

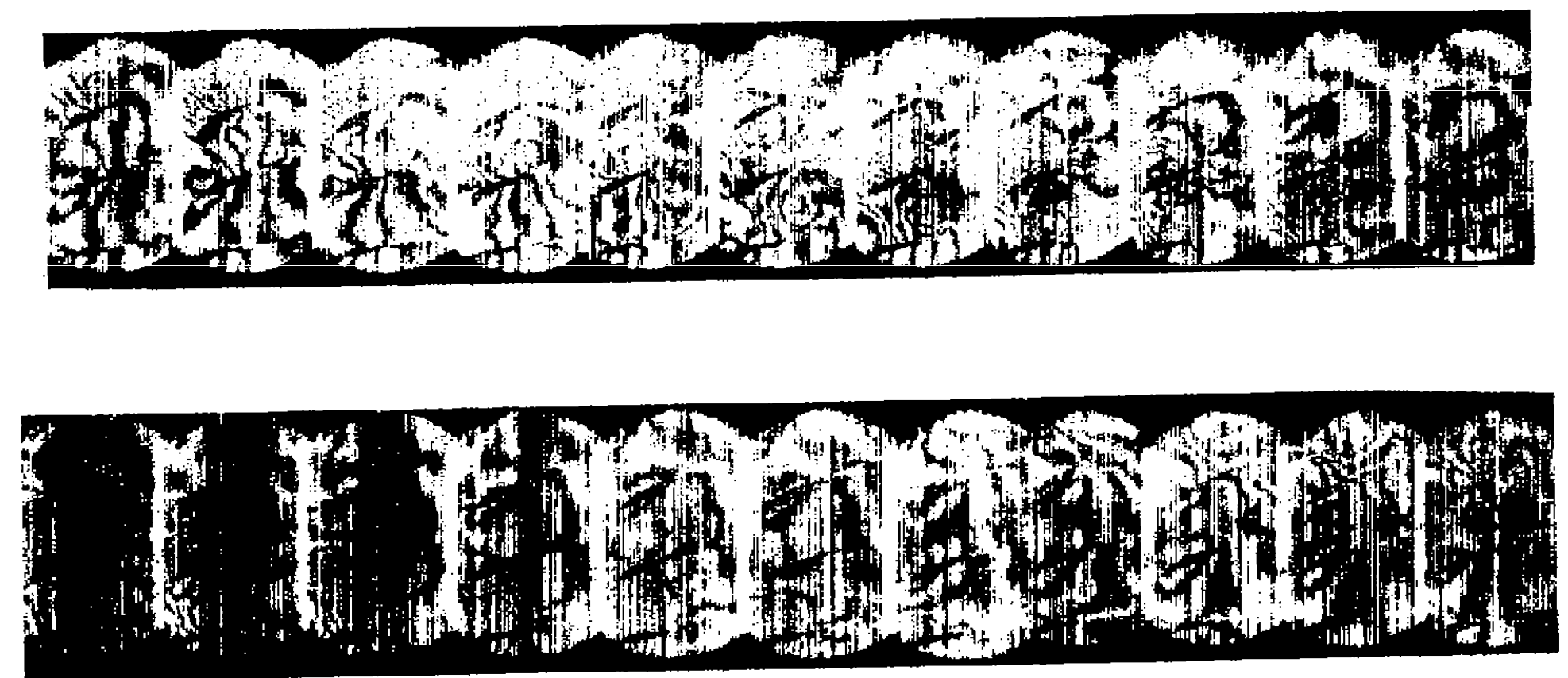

(b) $\beta_{1}=64^{\circ}$.

Figure 1.- Conclud̃ed. 


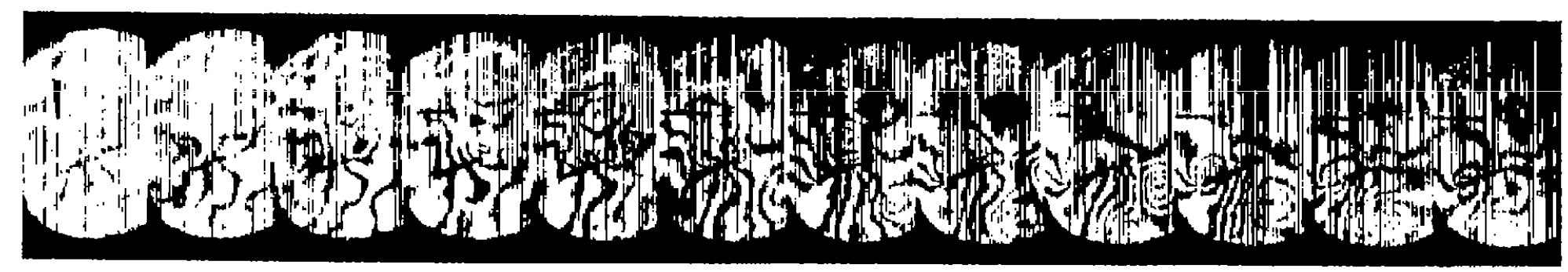

$$
\text { (a) } \beta_{1}=58^{\circ} \text {. }
$$

Figure 2.- Interferometer films. Configuration $B ; \sigma=1 / 2$. 

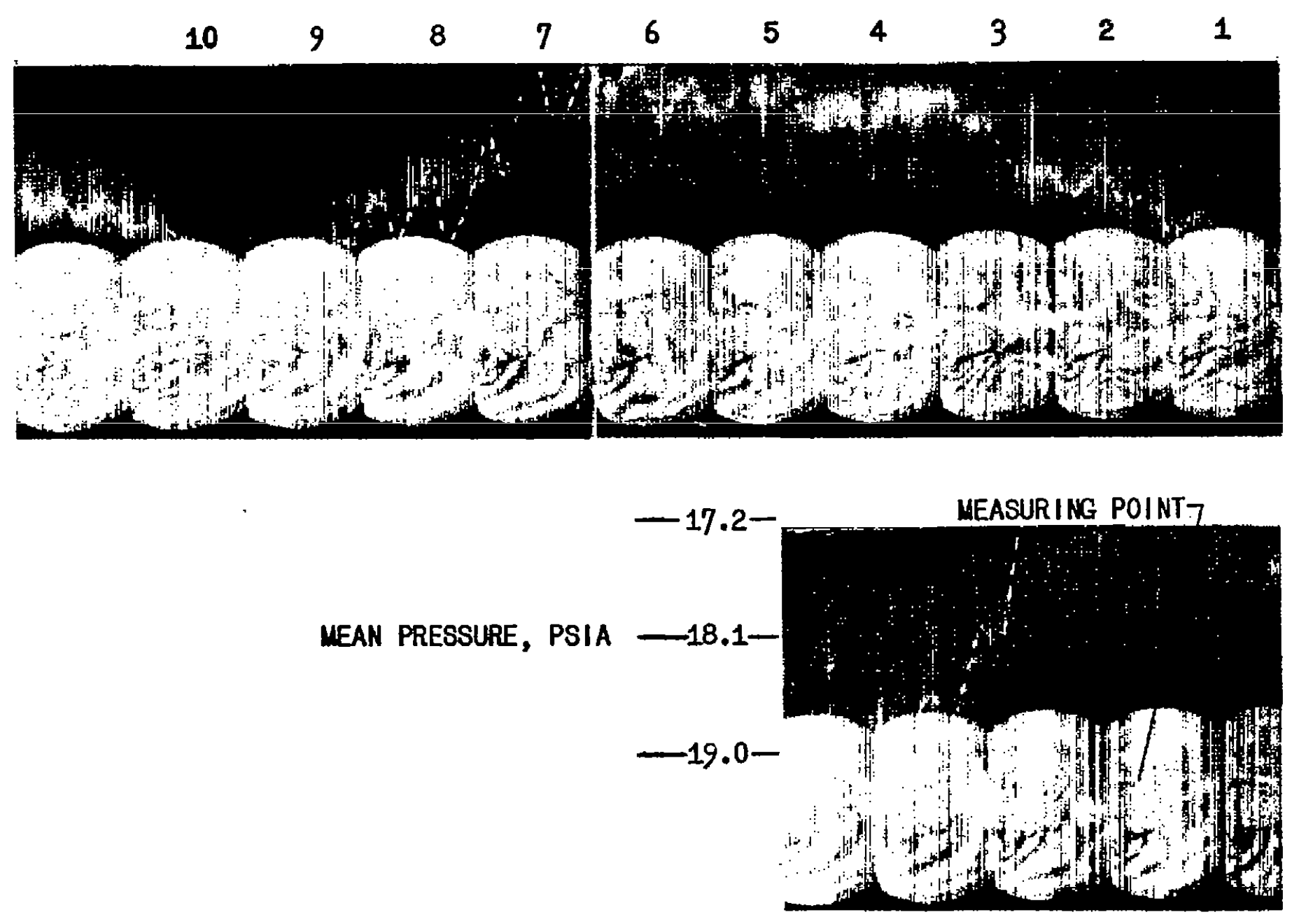

(b) $\beta_{1}=61^{\circ}$.

Figure 2.- Continued. 
崩

号
号
是
点
岌

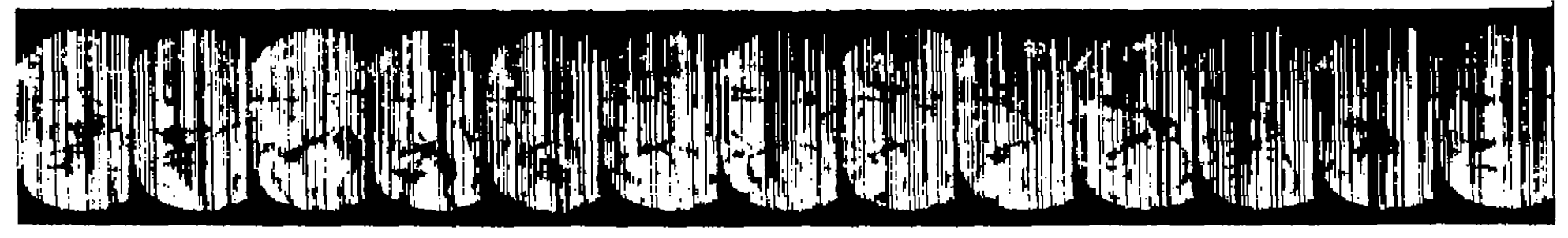

(c) $\beta_{1}=64^{\circ}$.

Figure 2.- Concluded. 


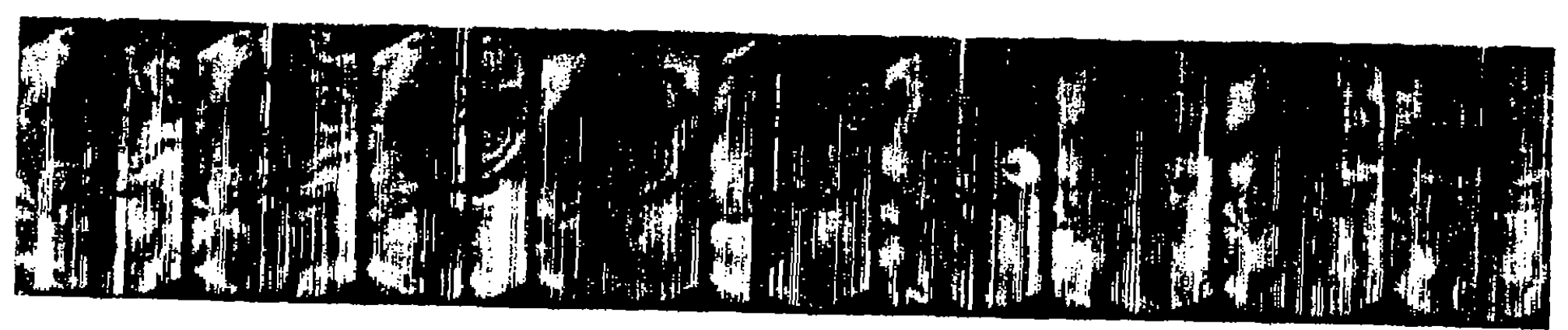

(a) $\beta_{1}=57^{\circ}$.

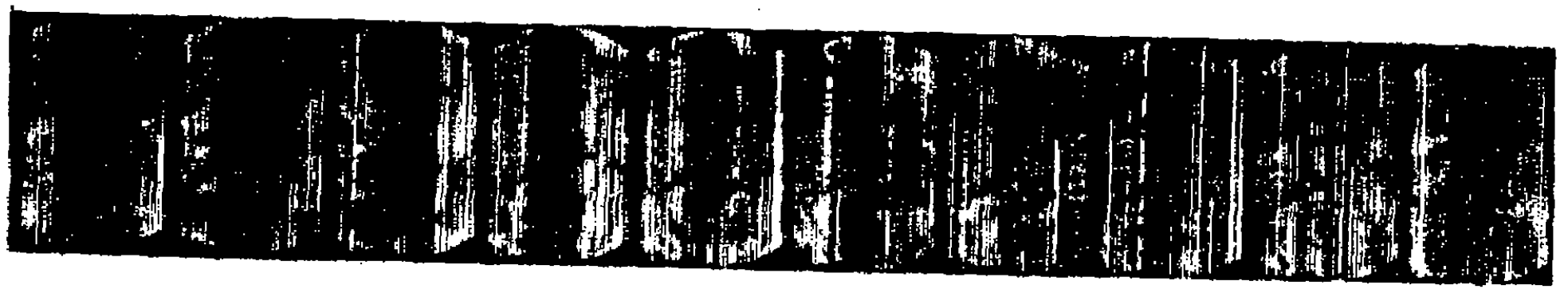

(b) $\beta_{I}=70^{\circ}$.

Figure 3. - Interferometer films. Configuration $B ; \sigma=1 / 3$.

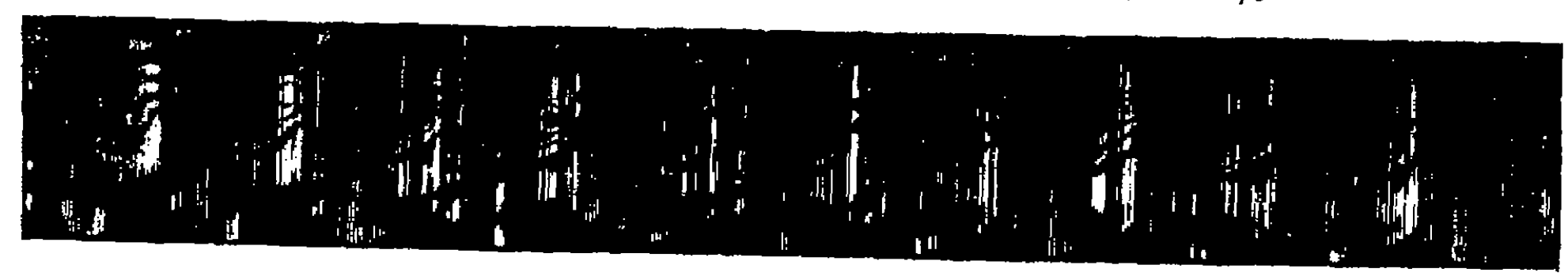

Figure 4.- Interferometer films. Configuration $B ; \sigma=1 / 6 ; \beta_{1}=57^{\circ}$. 


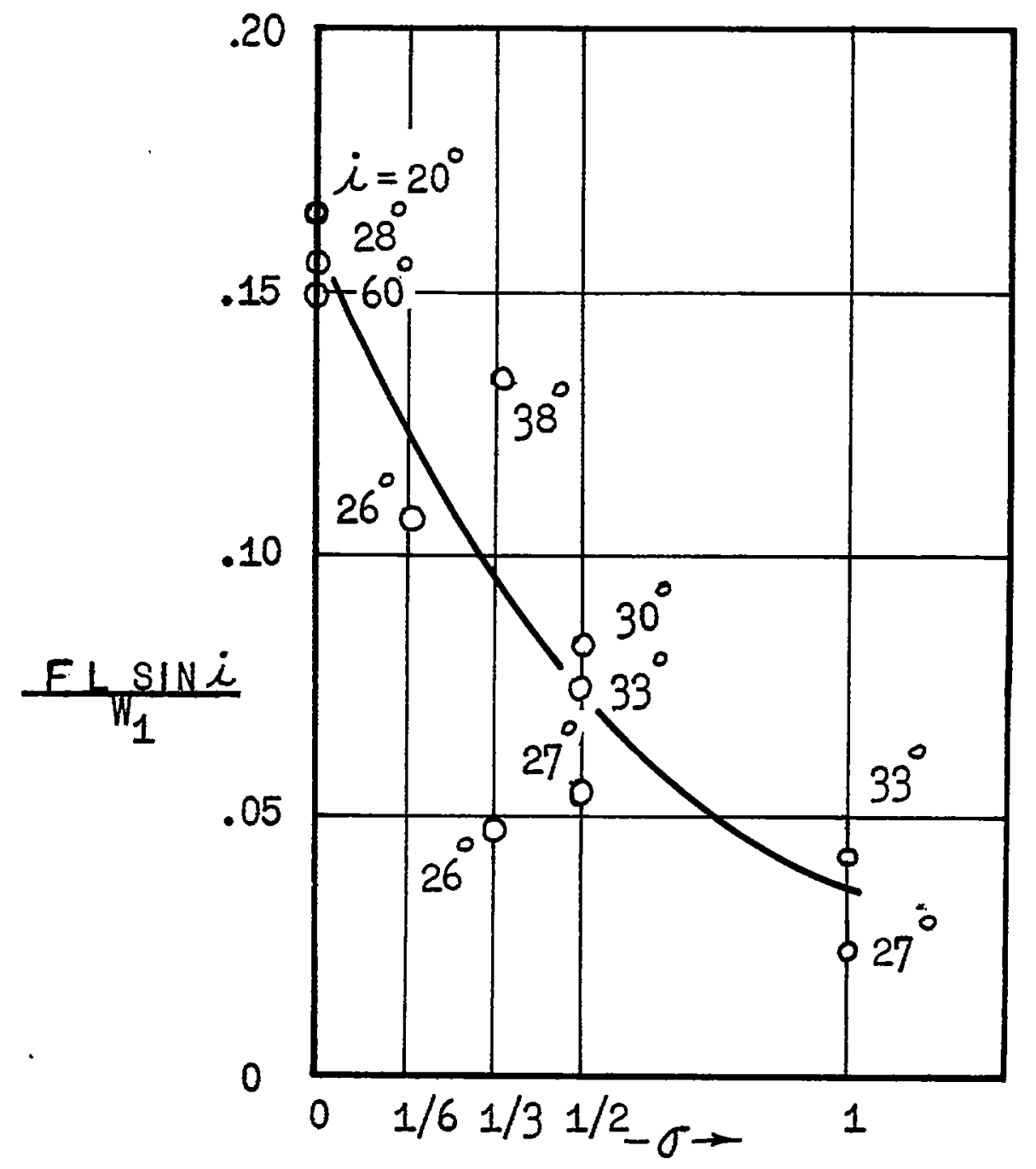

Figure 5.- Vortex shedding frequency against solidity. 


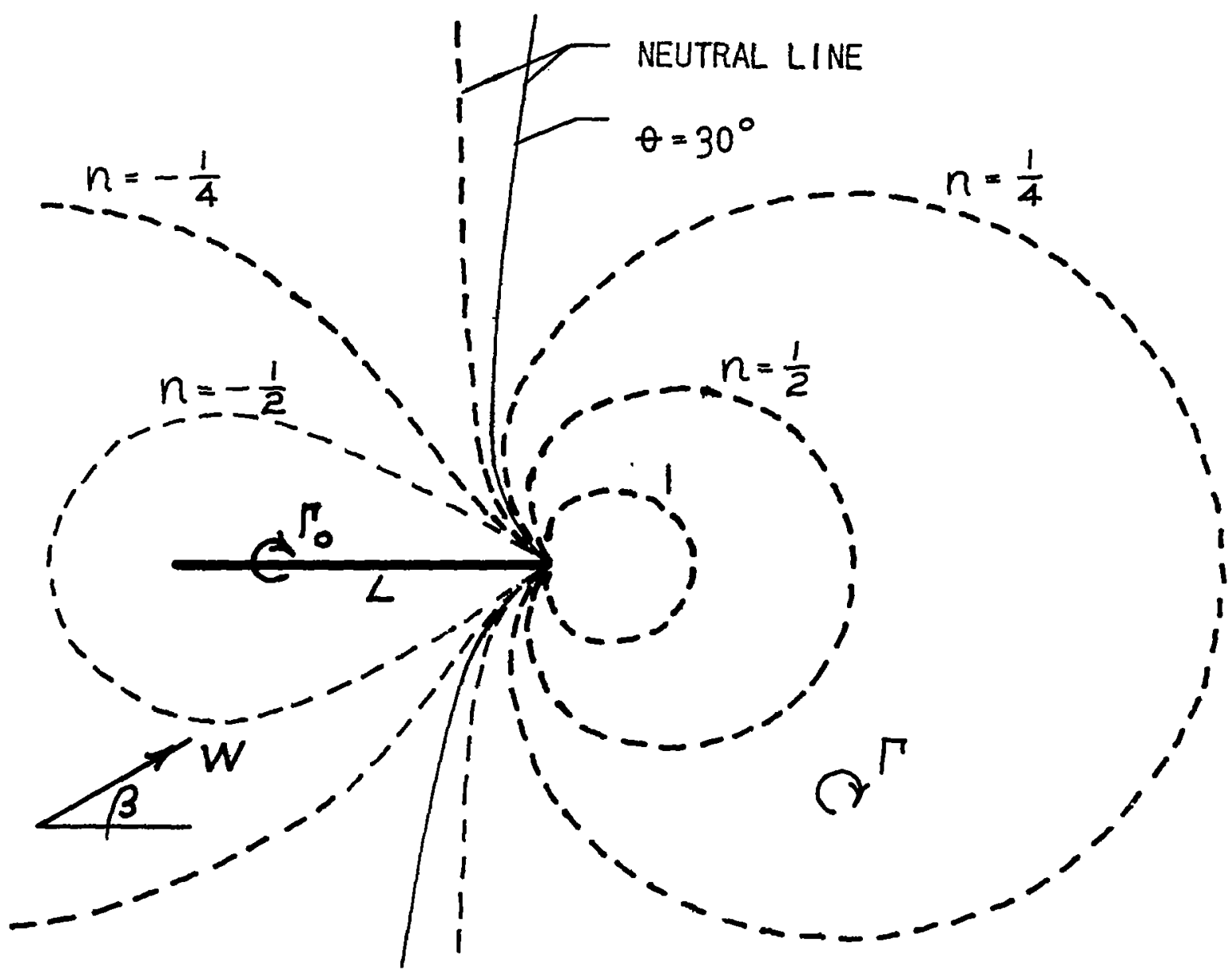

Figure 6.- Loc1 of vortex $\Gamma$ for constant plate circulation. 


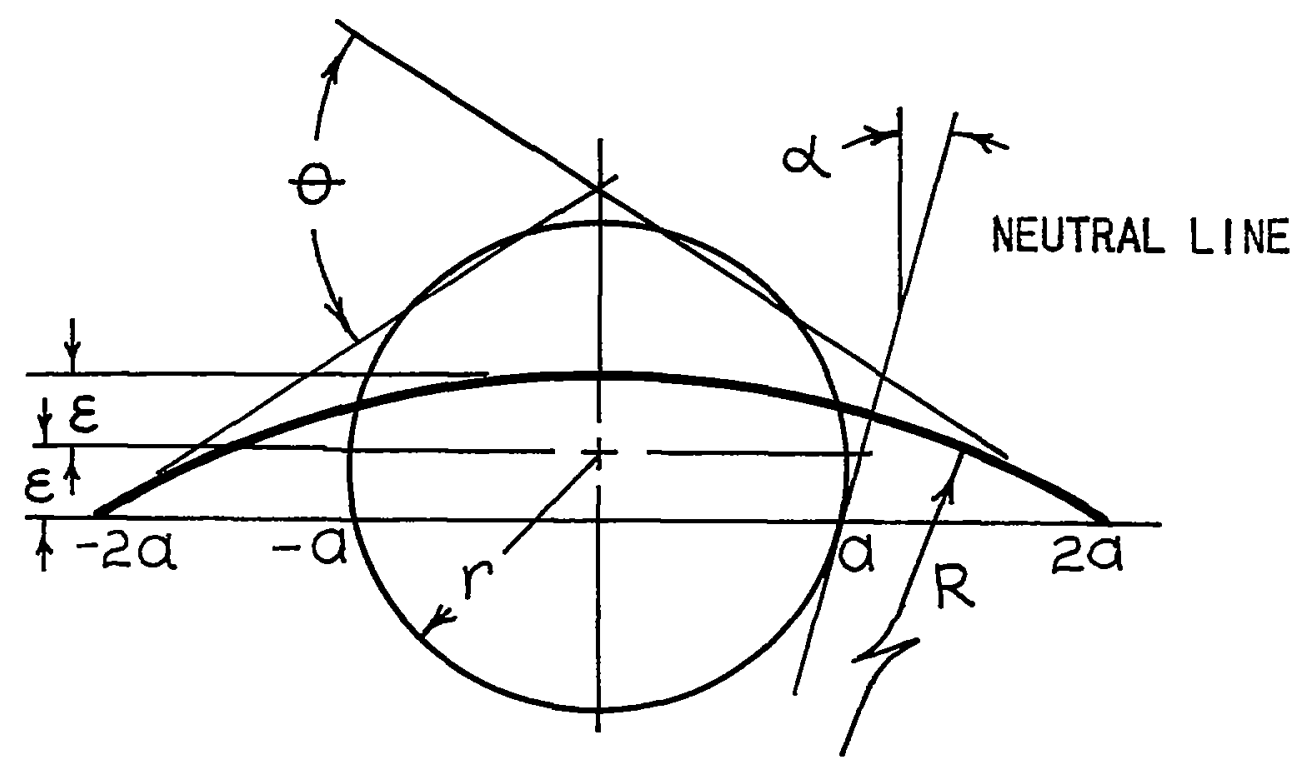

Figure 7.- Joukowski transformation of clrcular arc. 


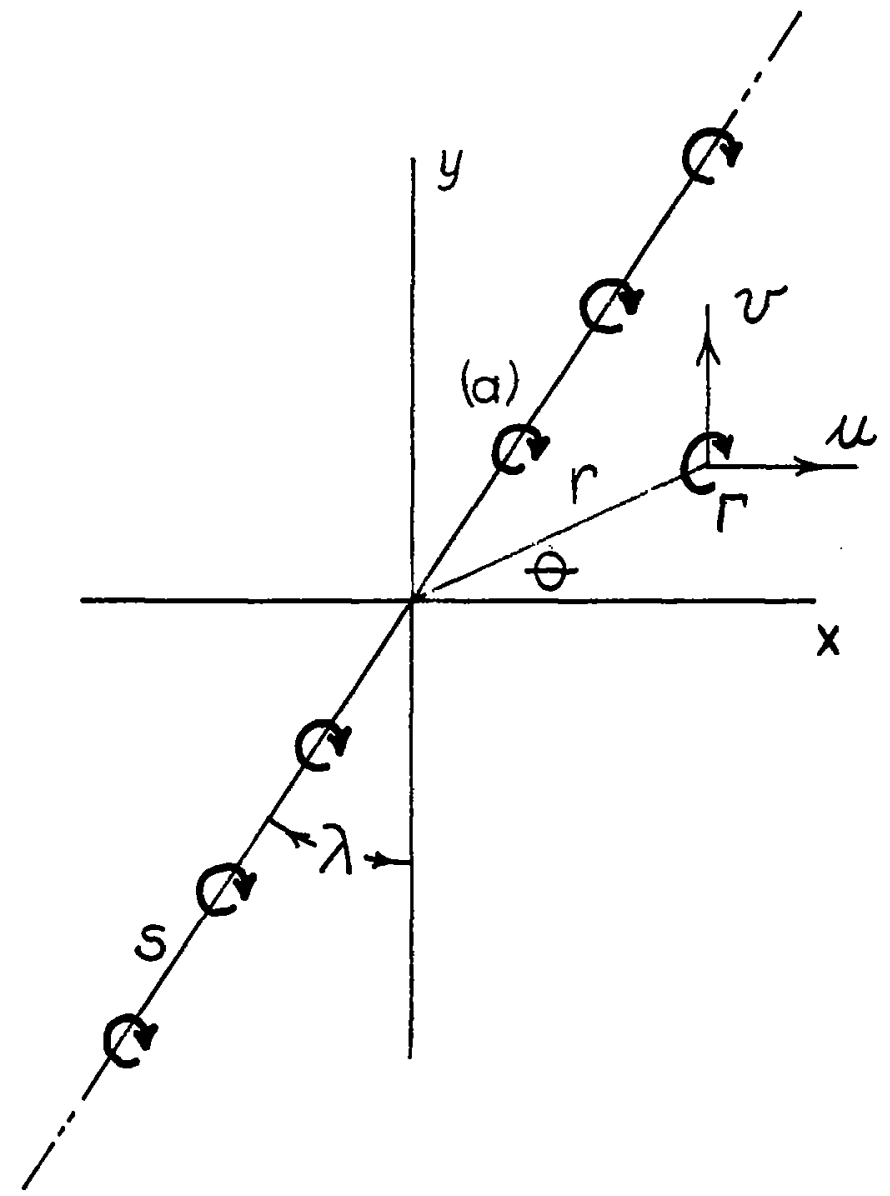

Figure 8.- Vortex representation of cascade. 


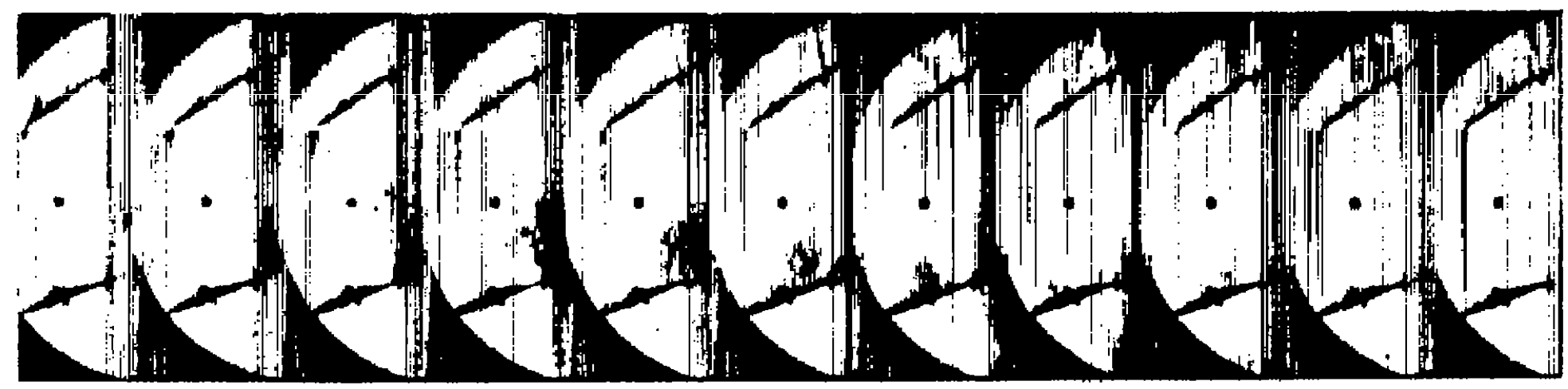

Figure 9.- Schlieren illin. Configuration $A ; \sigma=1 / 2 ; \hat{\beta}_{1}=60^{\circ}$. 


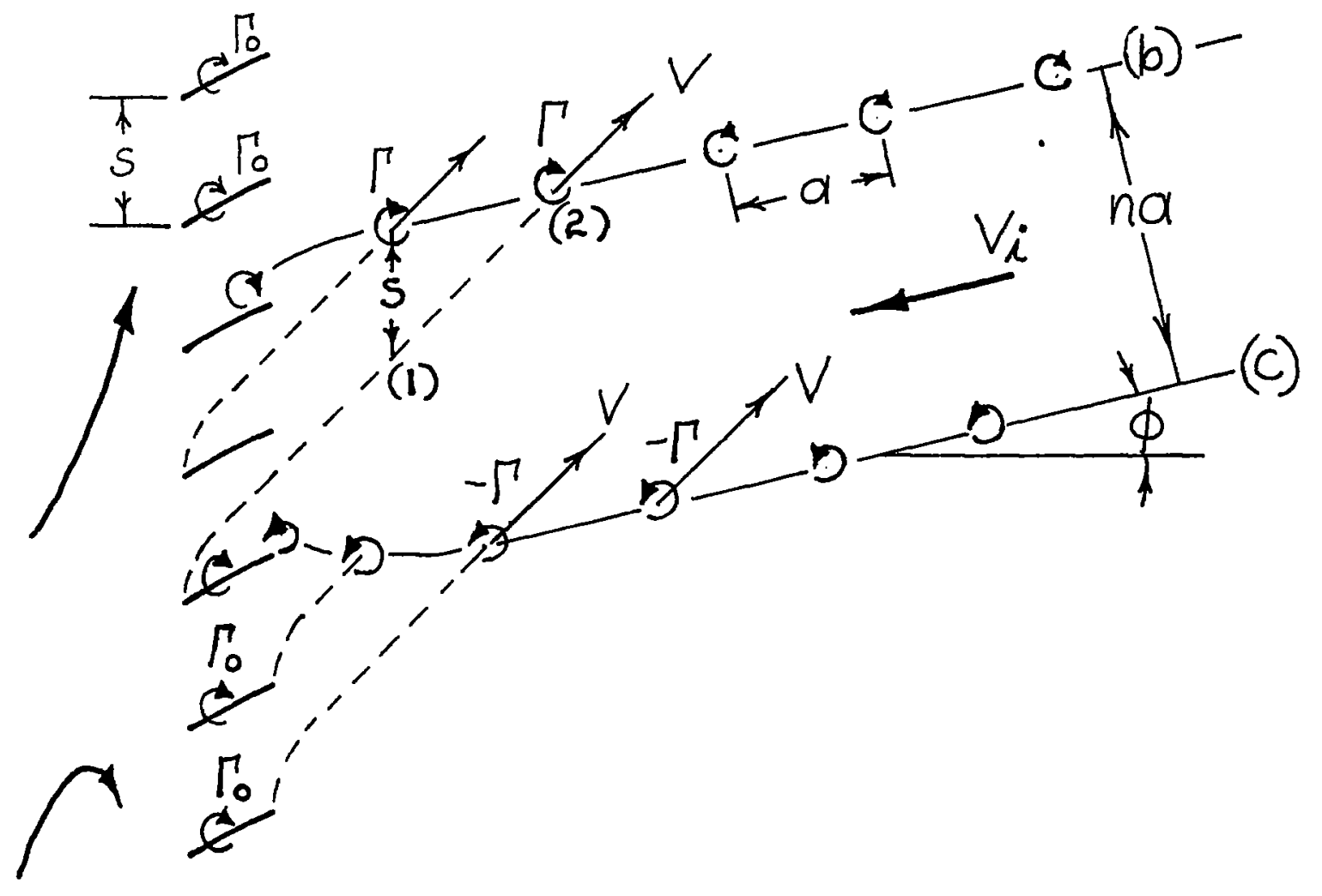

Flgure 10.- Pattern of vortices in stall cell. 


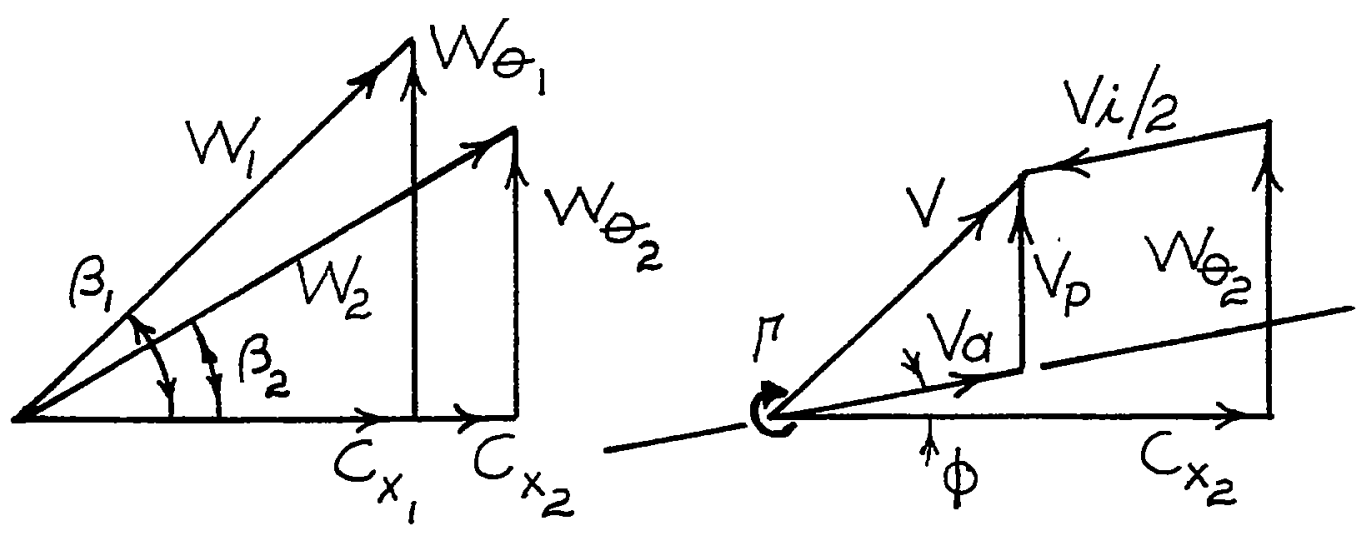

Flgure 1l.- Velocity triangles for stall cell. 


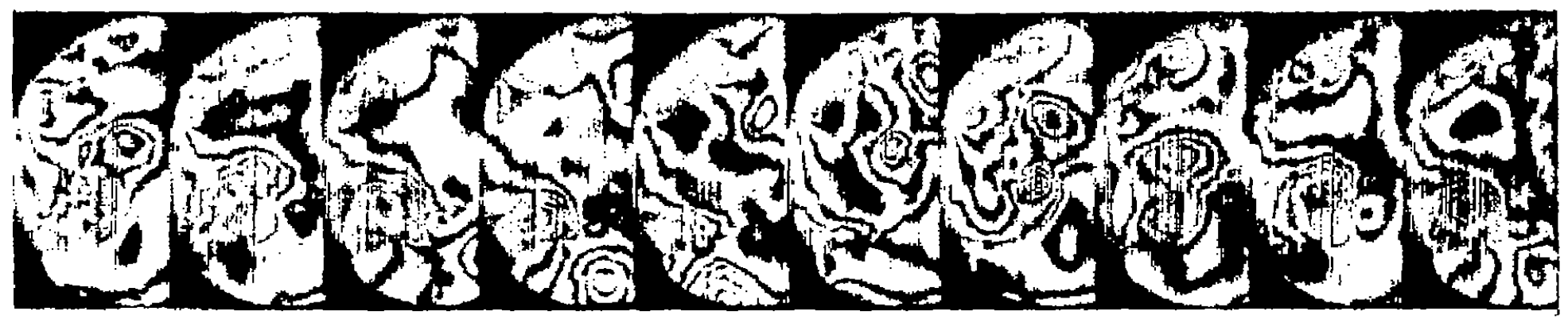

Figure 12.- Interferometer film. Configuration $A ; 8=1 / 2 ; \beta_{1}=60^{\circ}$. 


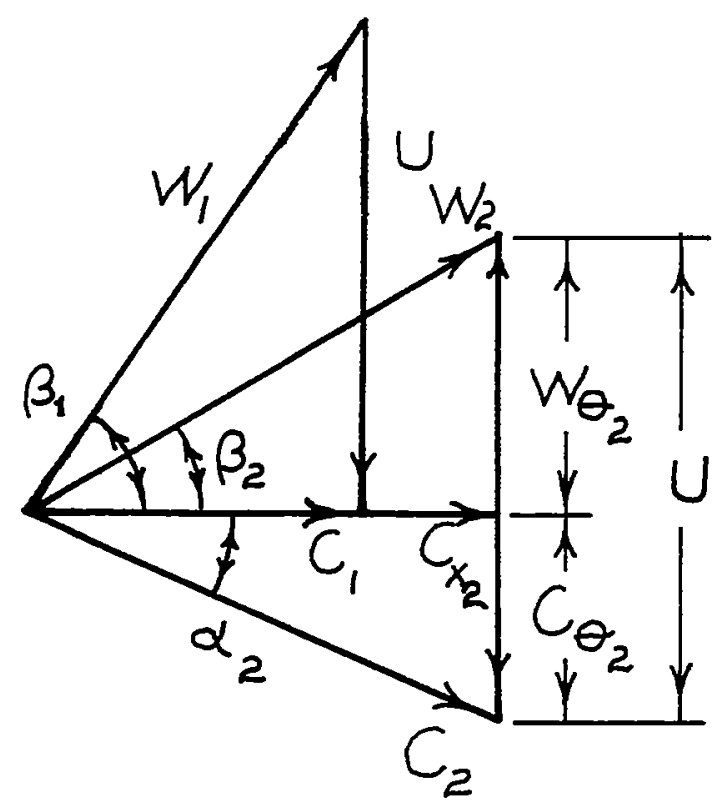

Figure 13.- Velocity triangles for isolated rotor. 


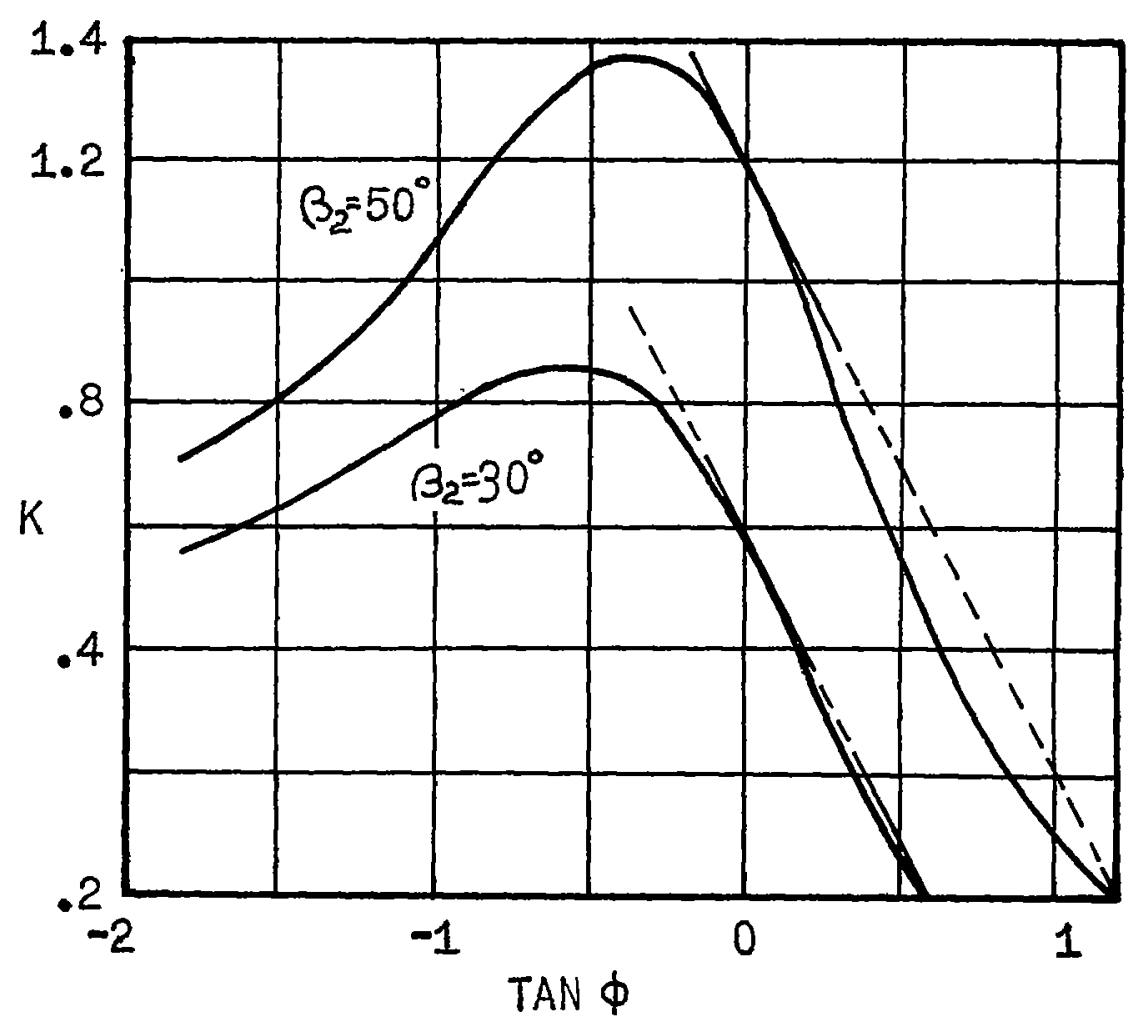

Flgure 14.- Plot of $K$ against $\tan \varnothing$ for two values of $\beta_{2}$. 


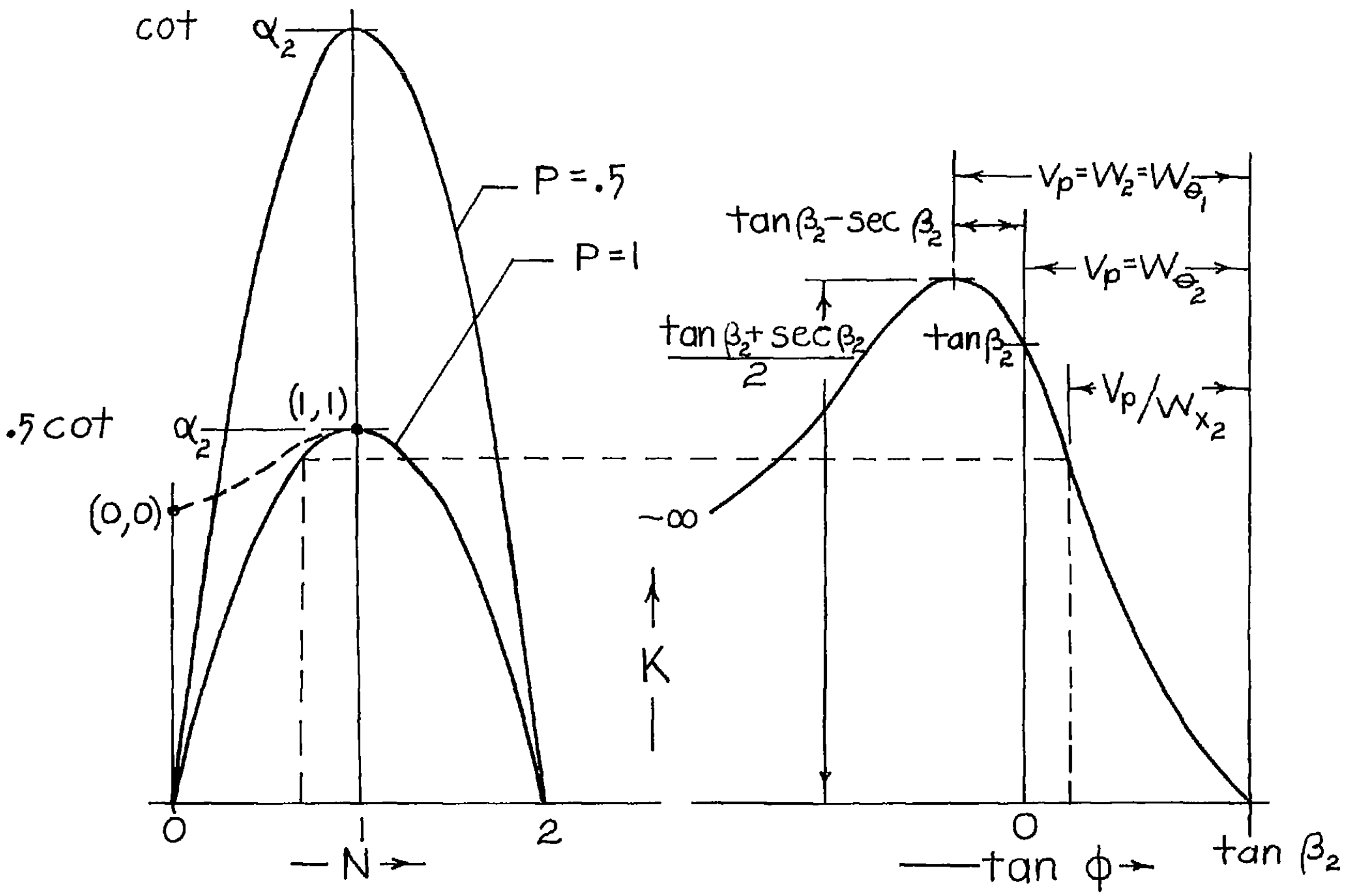

Figure 15.- Plot of $K$ against $P$ and $N$ and $\tan \phi$.

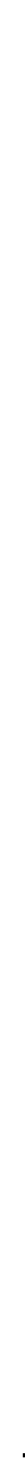




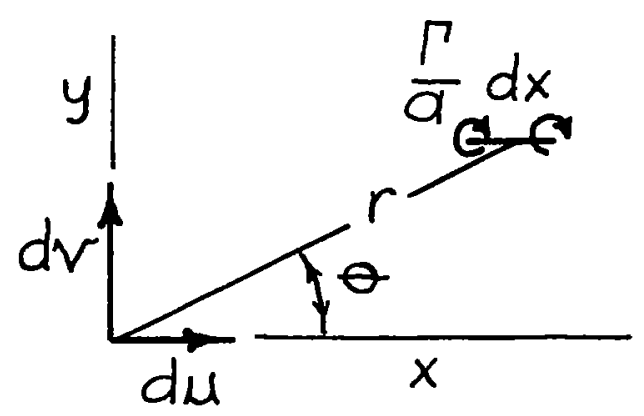

Figure 16.- Veloc1ty Induced from element of vortex sheet.

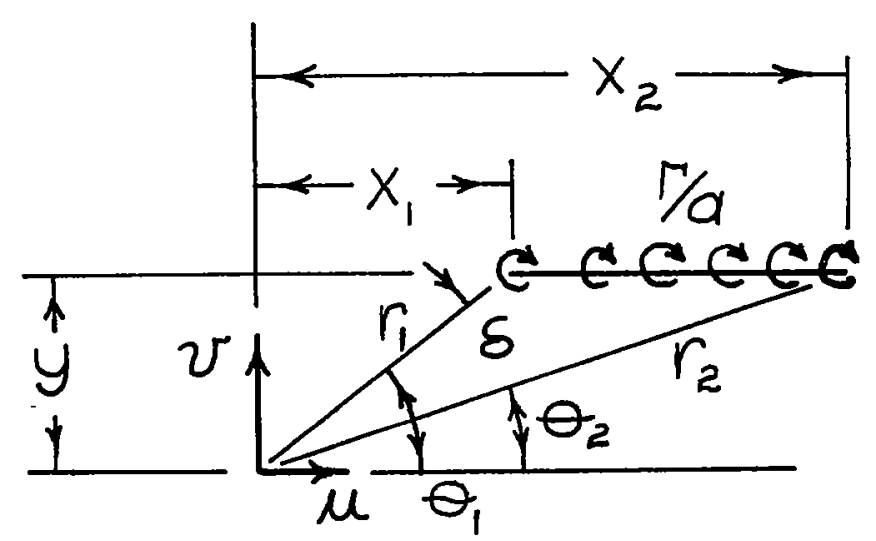

Flgure 17.- Velocity induced from finite-length vortex sheet. 


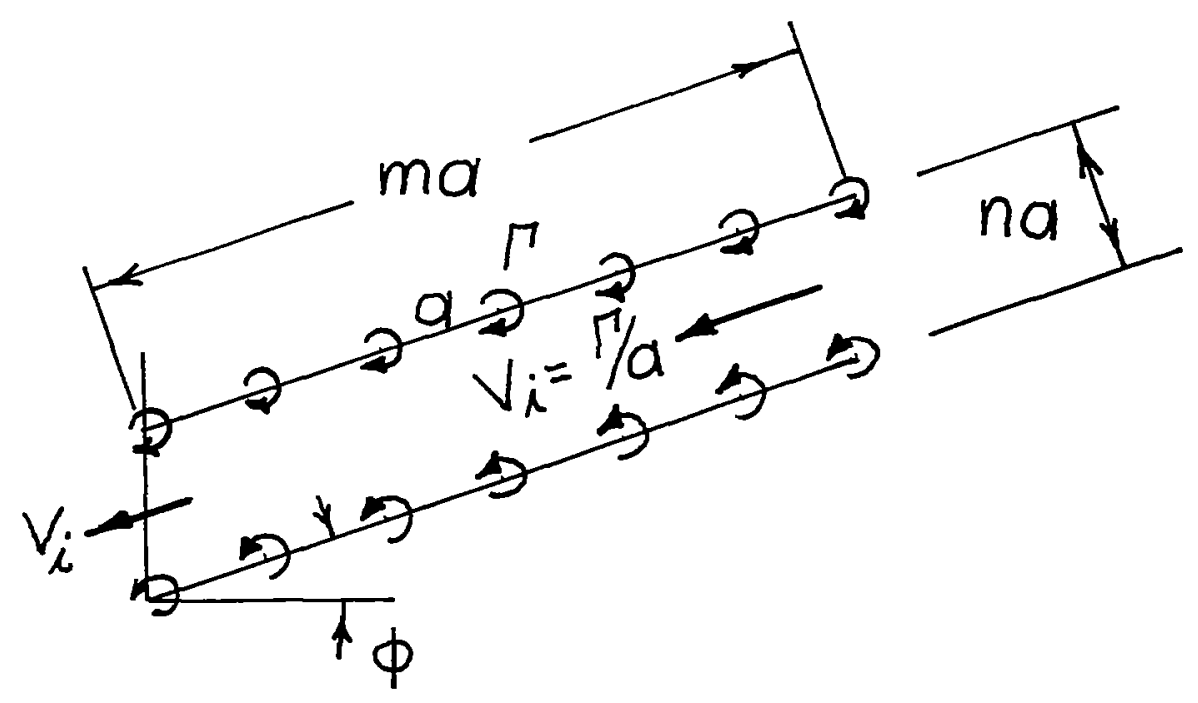

Figure 18.- Velocity induced from two finite vortex sheets at angle $\phi$. 


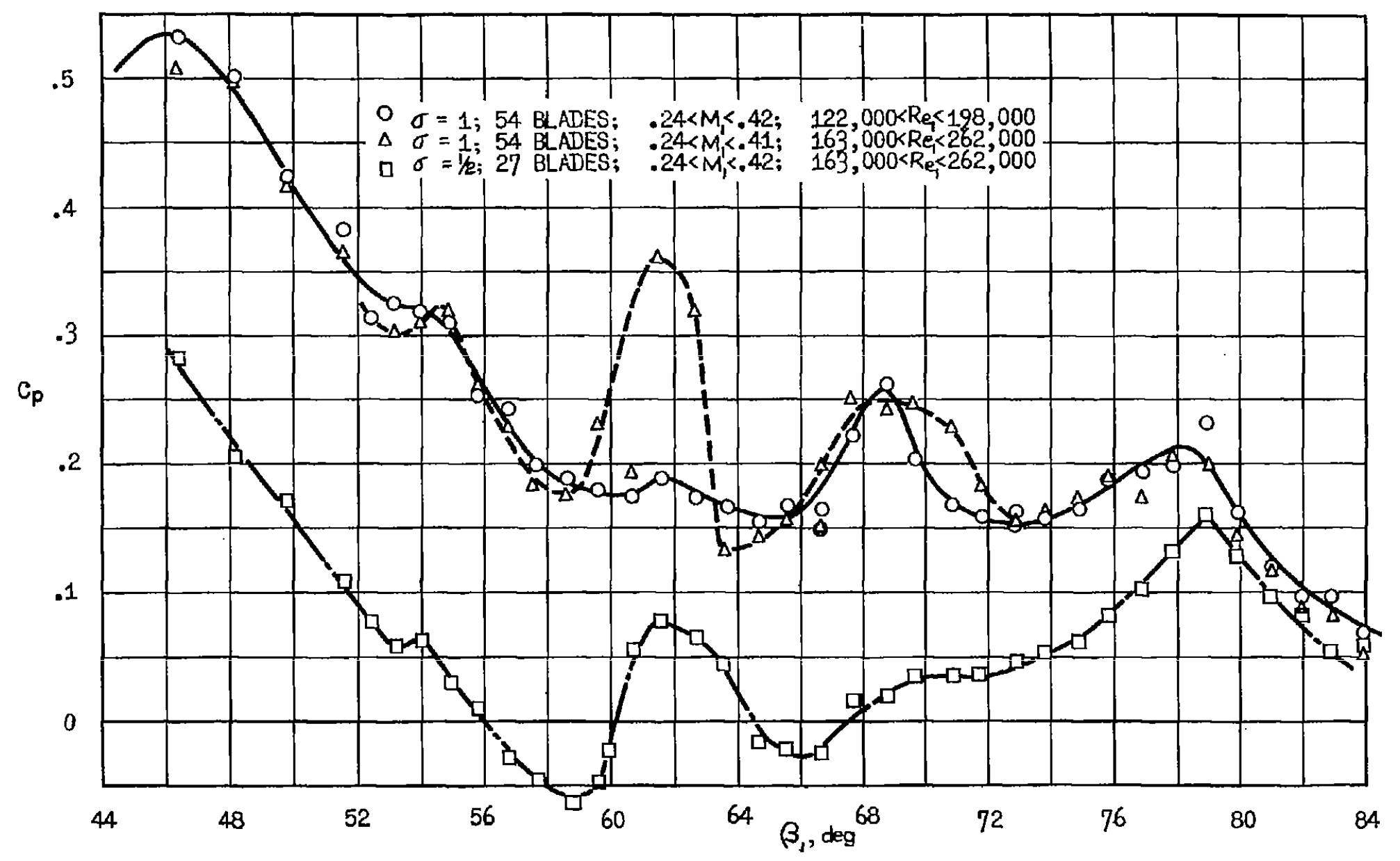

F1gure 19.- Plot of pressure coefficient against upstream air angle. Configuration A; values for $53^{\circ}<\beta_{1}<75^{\circ}$.

宫 


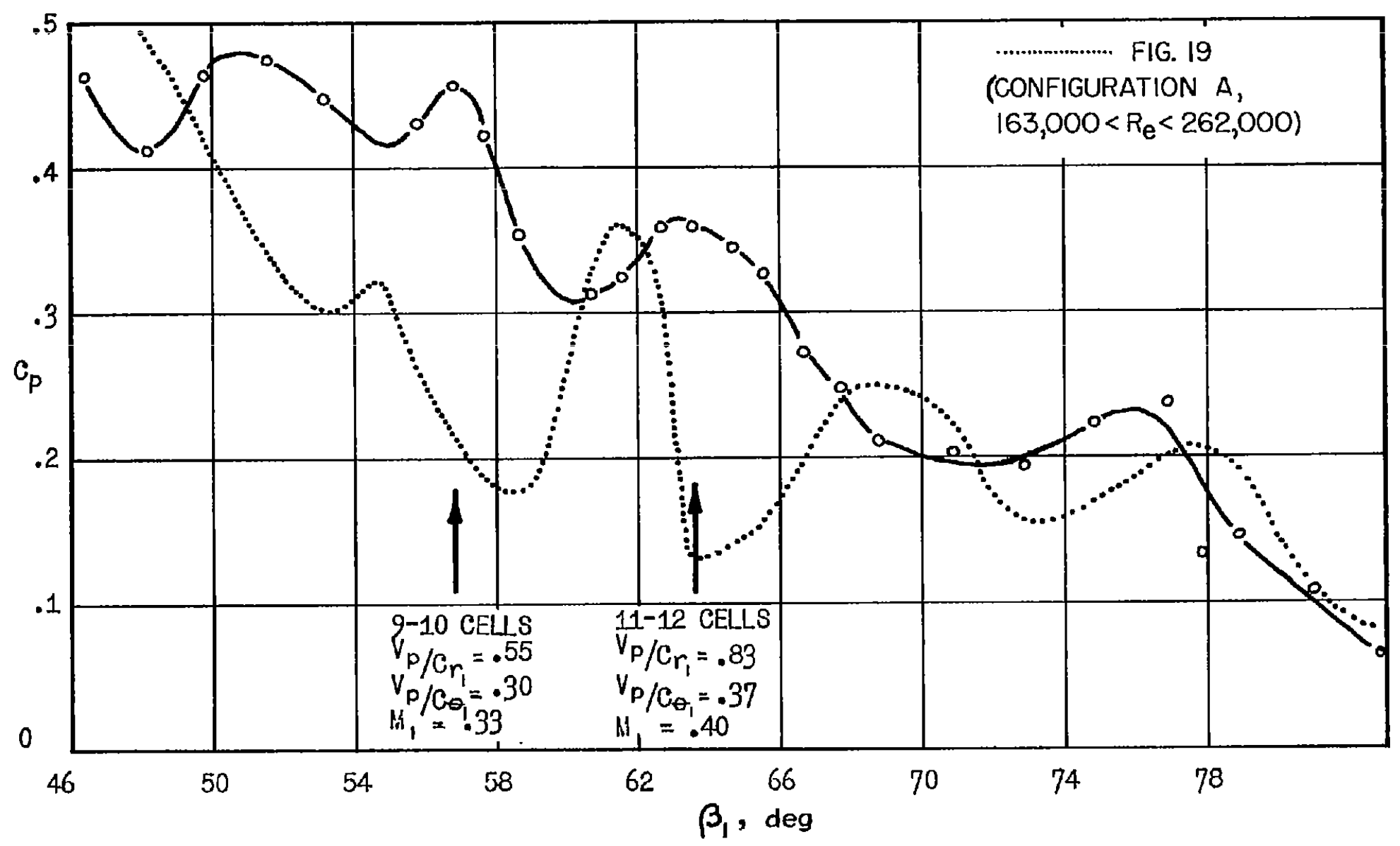

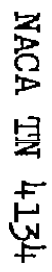

Figure 20.- Pressure coefficient against upstream air angle. Configuration B; $\sigma=1$; 54 blades; $53^{\circ}<\beta_{1}<75^{\circ} ; 0.28<M_{1}<0.45 ; 215,000<R_{e_{1}}<350,000$.

$\stackrel{\infty}{\infty}$ 


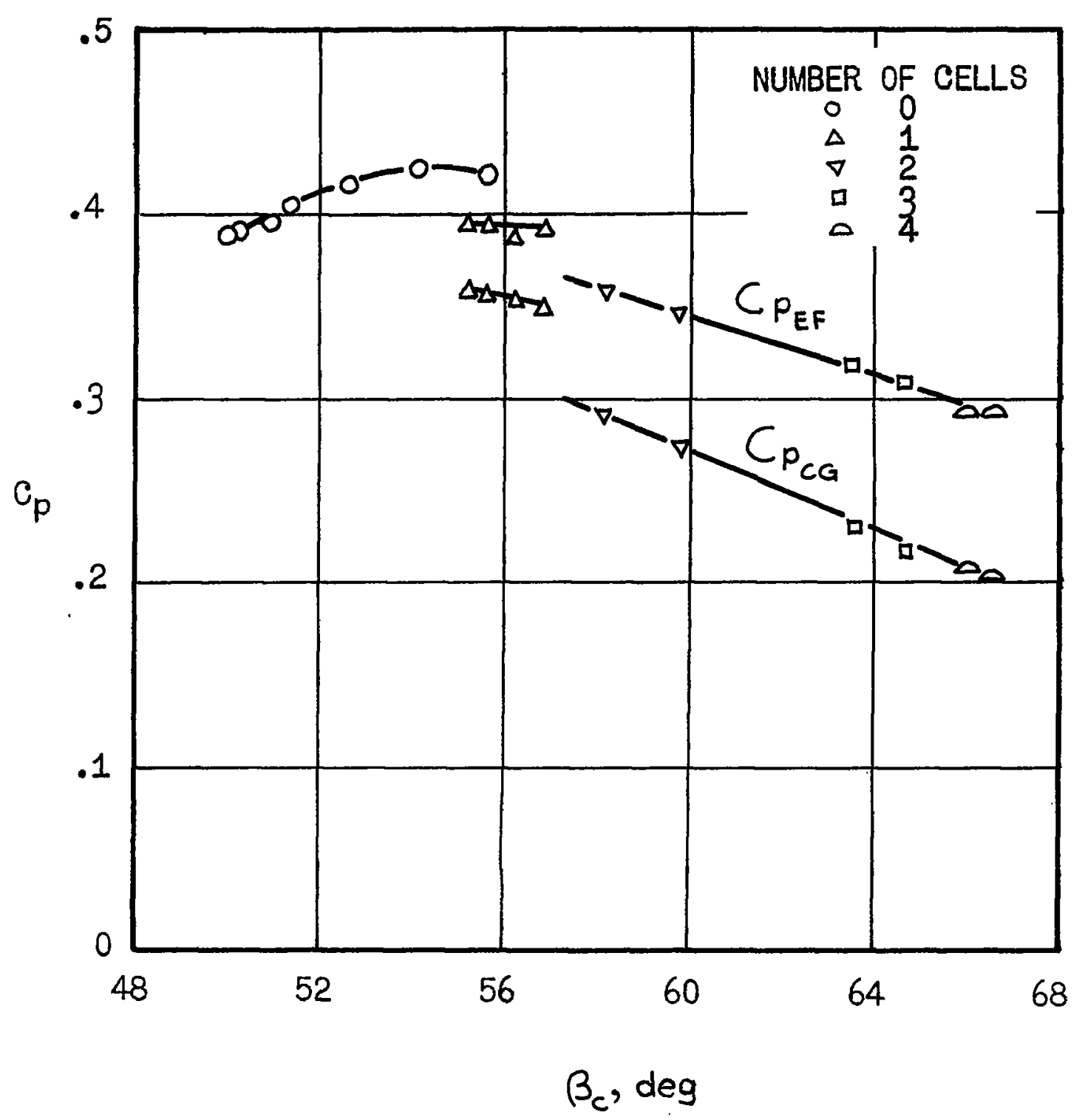

F1gure 21.- Pressure coefficient against $\beta_{C}$. Configuration A. 


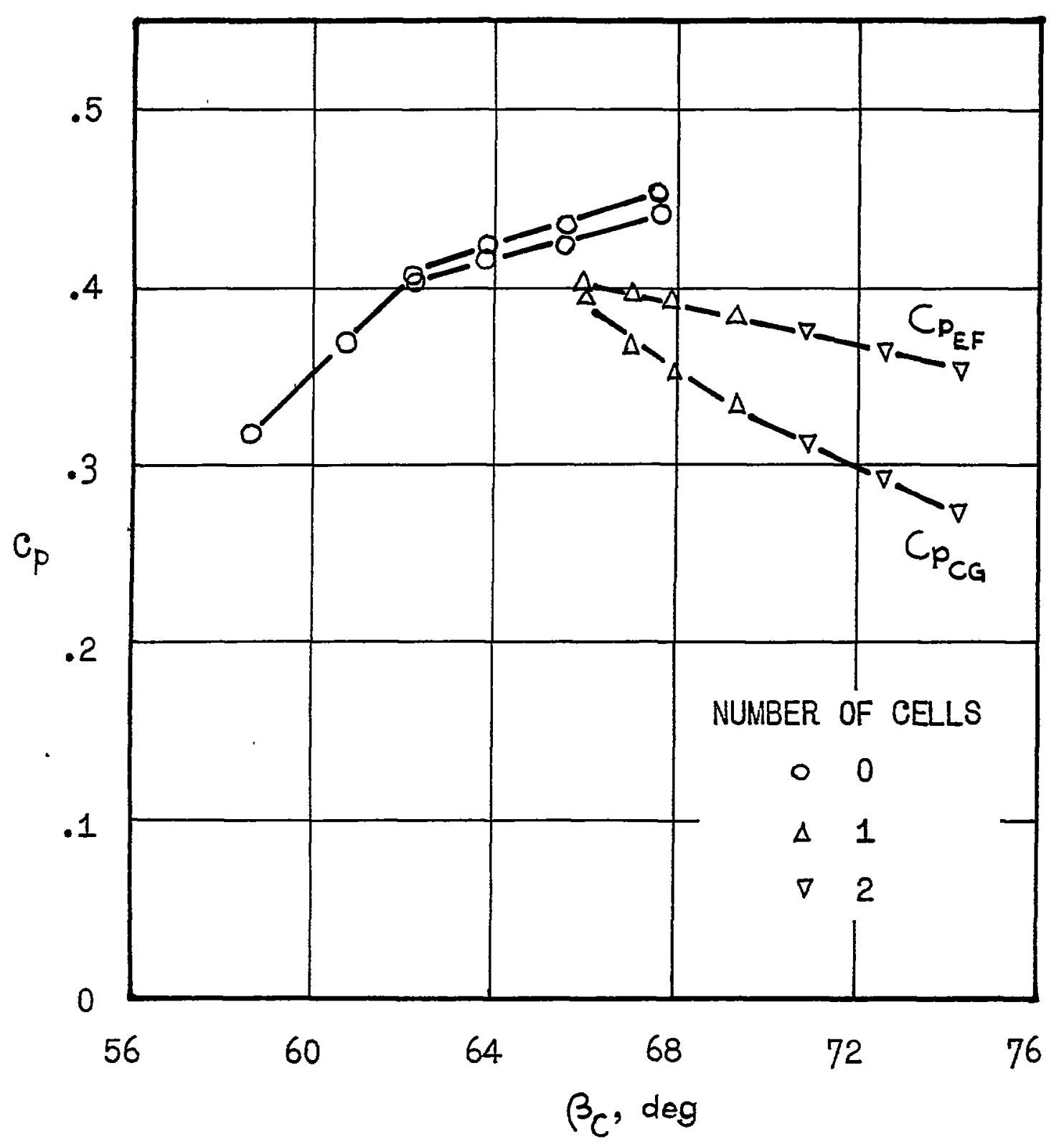

Figure 22.- Pressure coefficient versus $\beta_{C}$. Configuration $C$. 


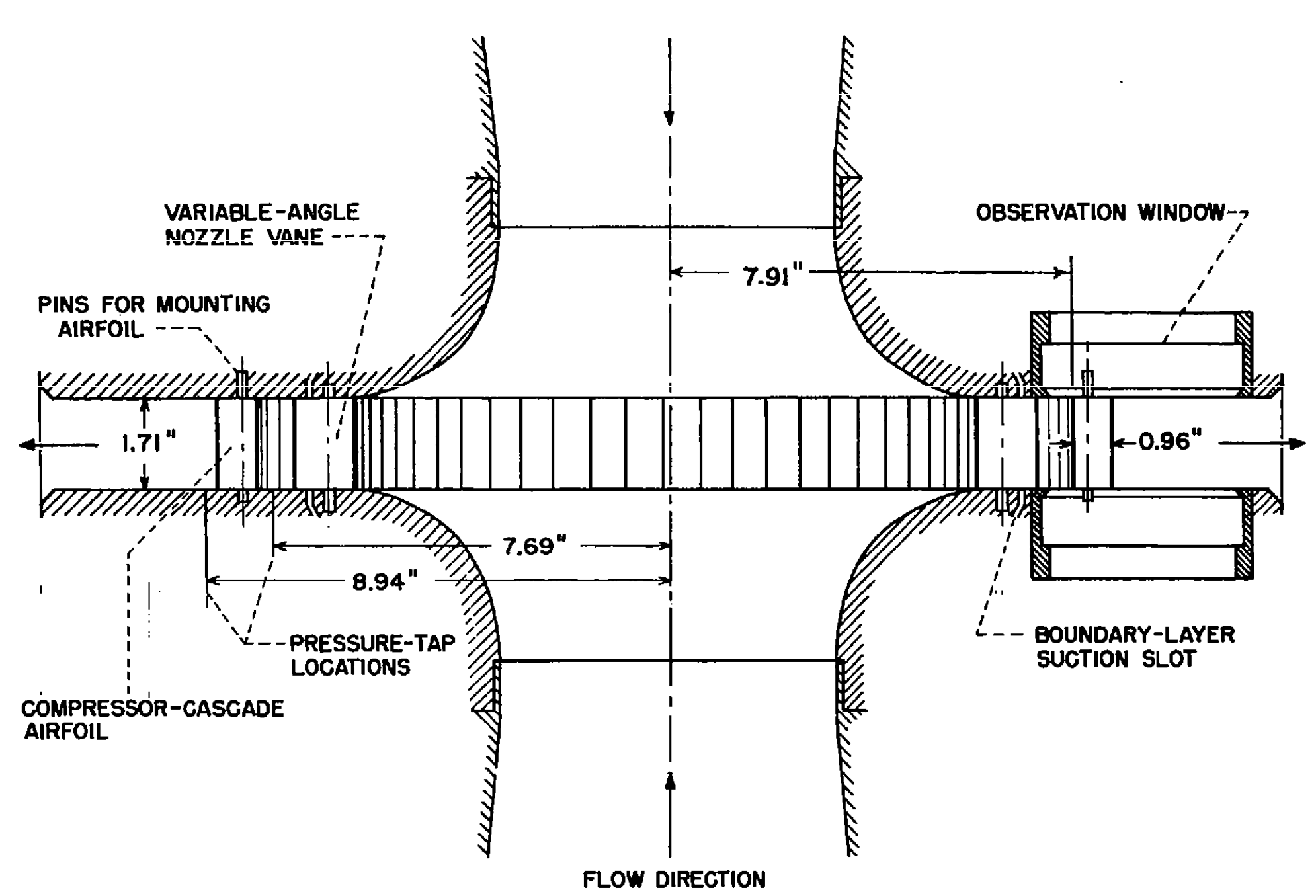

Figure 23.- Schematic diagram of circular-cascade test section. Conflguration A. 


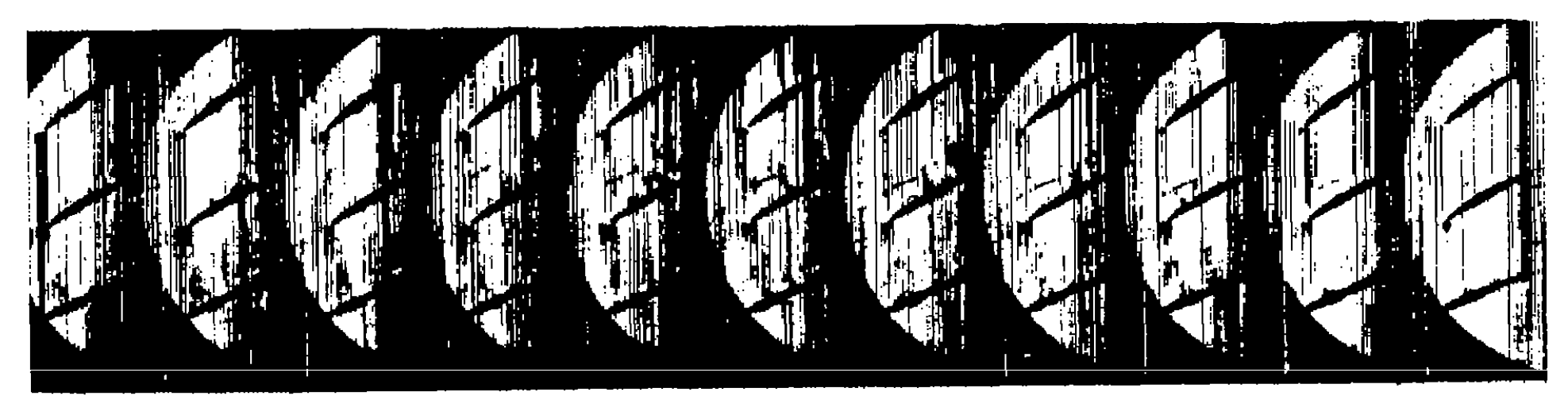

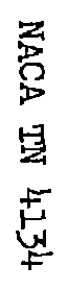

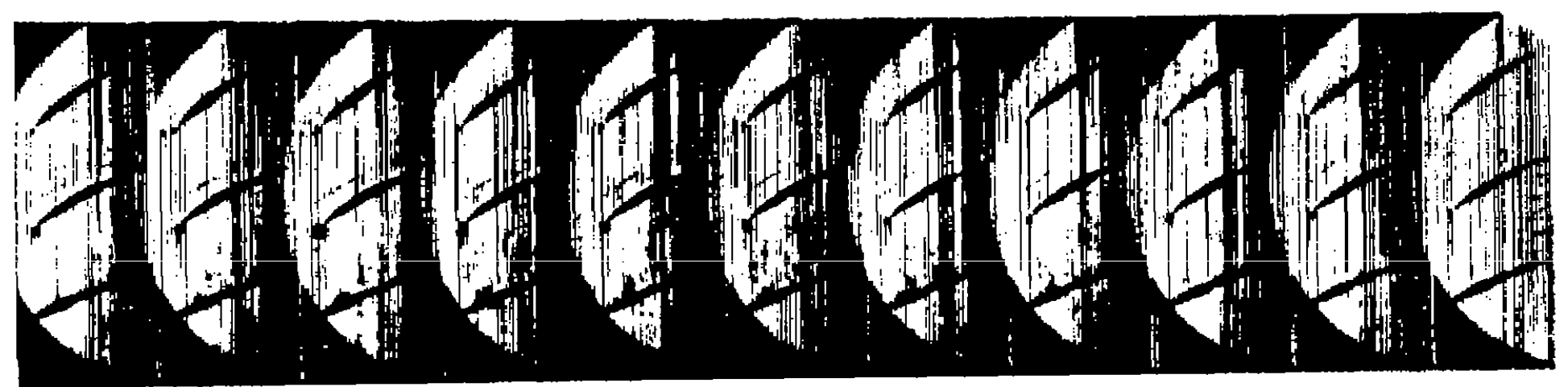

(a) $\beta_{1}=55^{\circ}$.

Figure 24.- Schlieren films. Configuration A; $\sigma=1$; time is from right to left. 

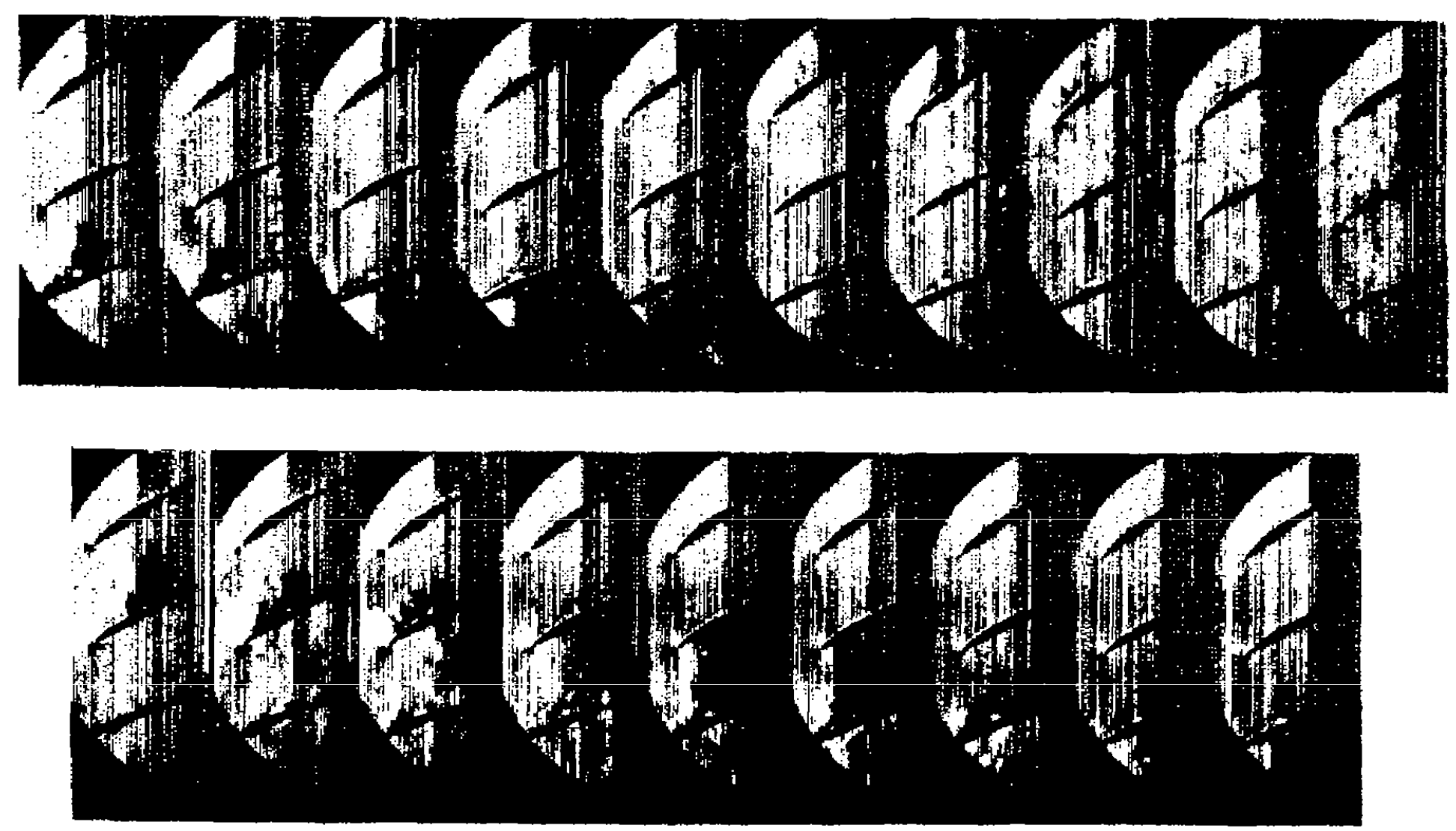

(b) $\beta_{I}=60^{\circ}$.

唆
薏
点

Figure 24.- Continued. 


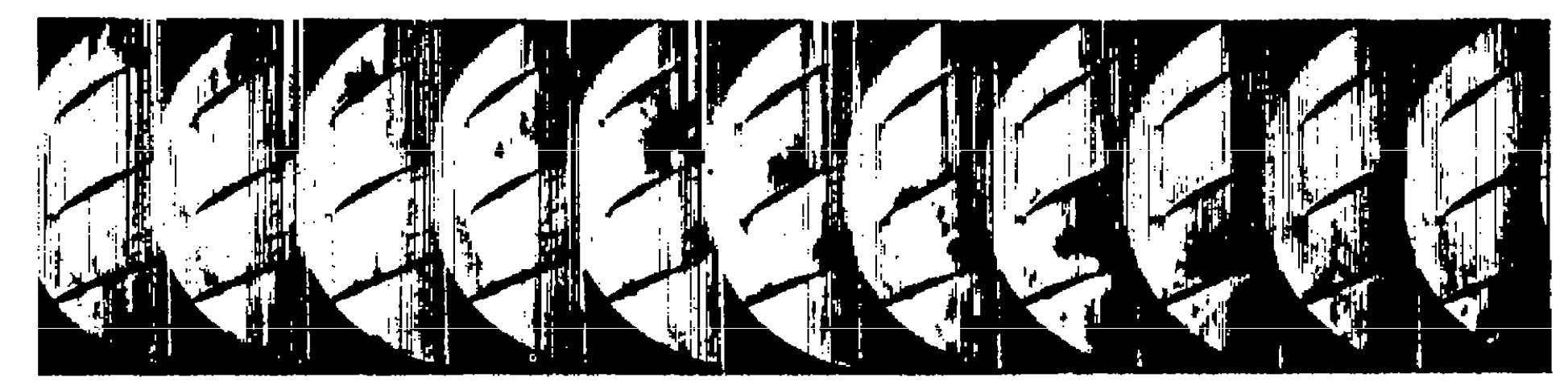

(c) $\beta_{1}=68^{\circ}$.

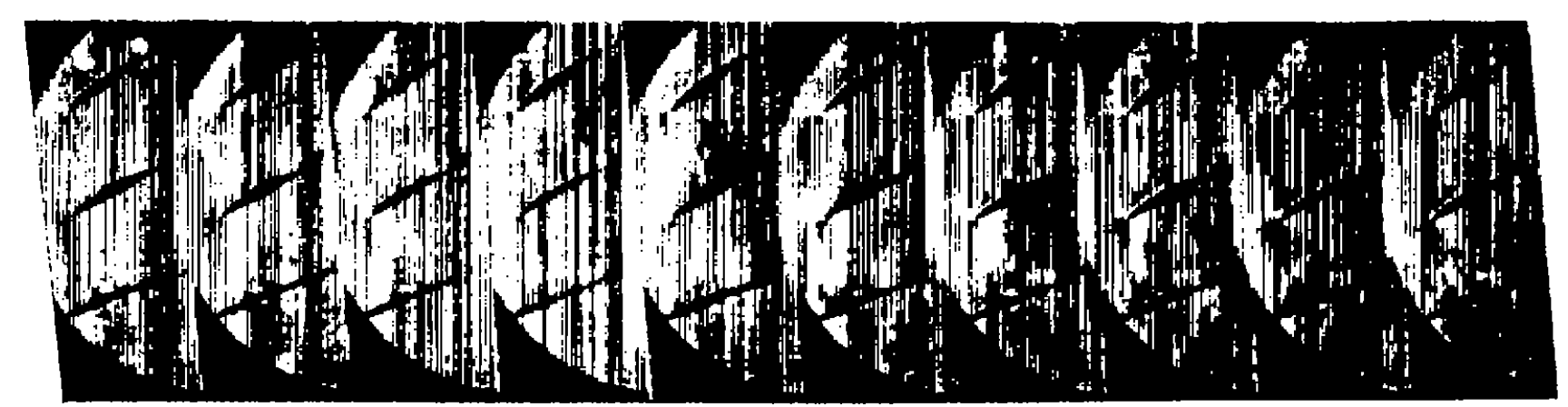

(d) $\beta_{1}=81^{\circ}$.

Figure 24.- Concluded. 


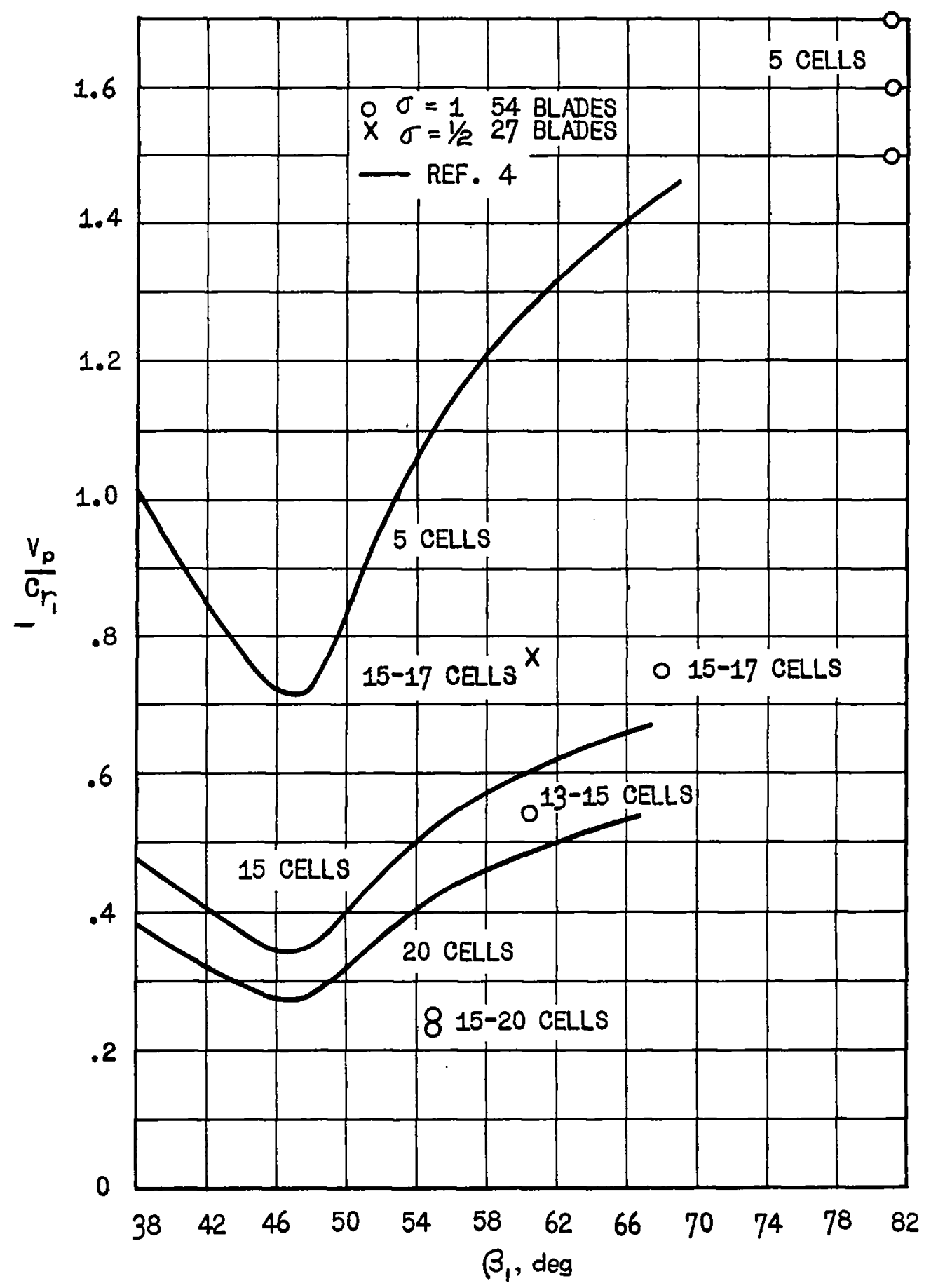

Figure 25.- Velocity of stall propagation against upstream air angle. Configuration $\bar{A}$. 
A

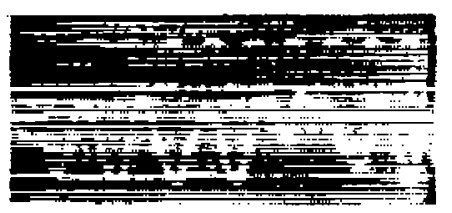

\begin{tabular}{cccc}
$\beta_{1}$, & $\sigma$ & CONFIGURATION & $\begin{array}{c}\text { TIMINING } \\
\text { FREQUENCY } \\
\text { CPS }\end{array}$ \\
\hline 55 & 1 & A & 200
\end{tabular}

B

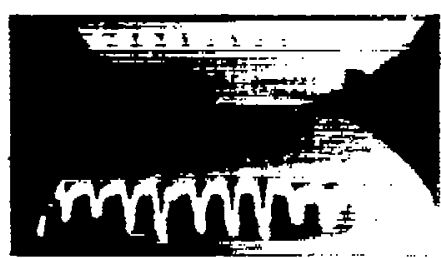

631

A

200

C

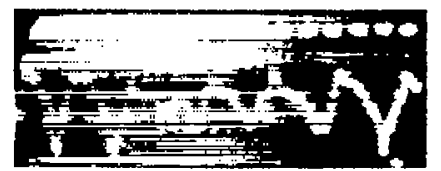

691

A

1,000

D

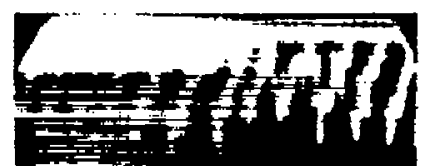

811

A

400

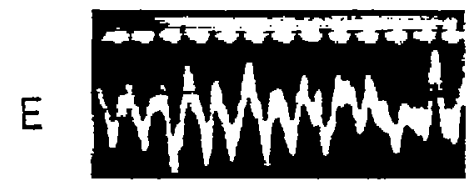

$611 / 2$

A

500

F

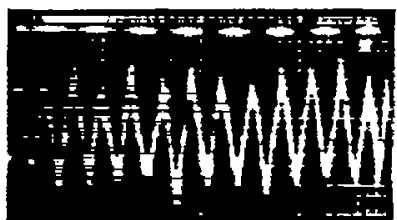

$571 / 3$

B

500

G

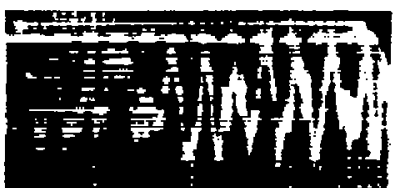

$70 \quad 1 / 3$

B

500

Figure 26.- Hot-wire traces upstream of circuiar cascade. 

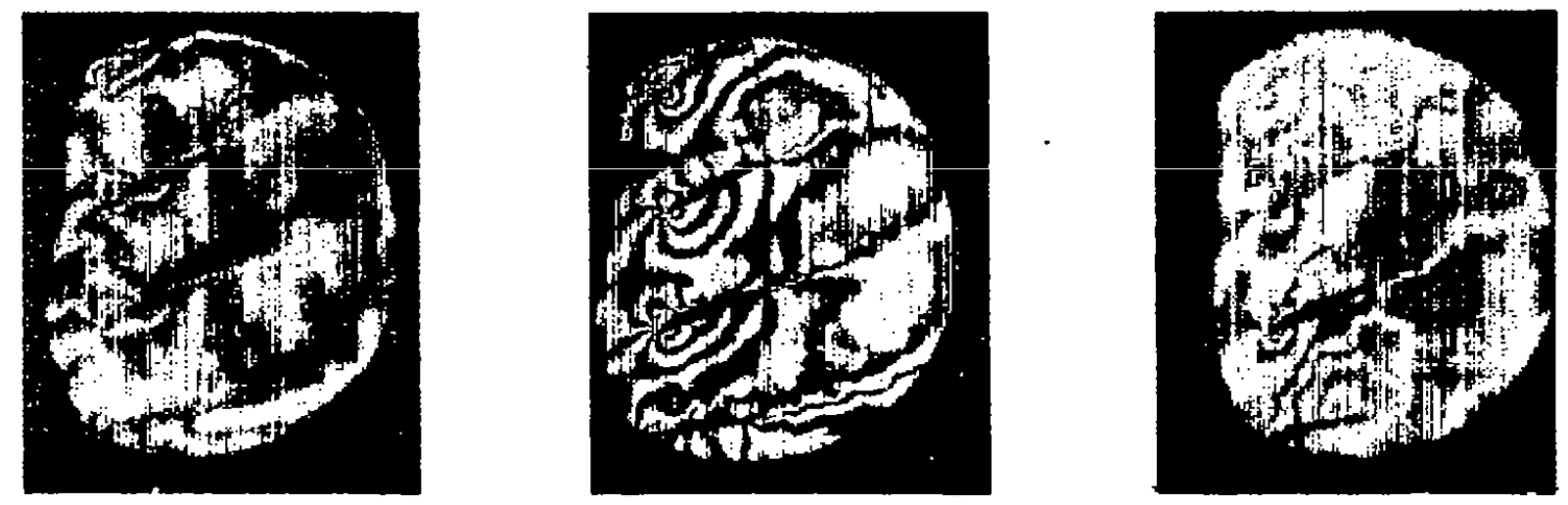

(a) $\beta_{1}=46.4^{\circ} ; M_{1}=0.45$.

(b) $\beta_{1}=51.6^{\circ} ; M_{1}=0.50$.

(c) $B_{1}=60.7^{\circ} ; M_{1}=0.38$.

Figure 27.- Interferograms of steady flow. Configuration B; $\sigma=1$. 


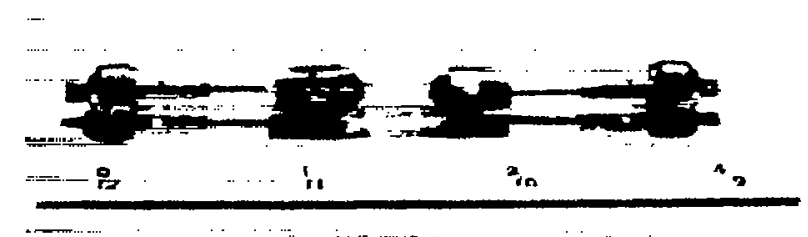

F1gure 28.- Photograph of light source.

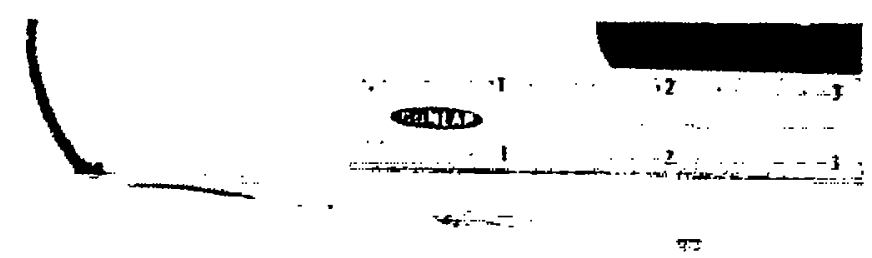

Figure 29.- Photograph of piezoelectric crystal pressure pickup. 


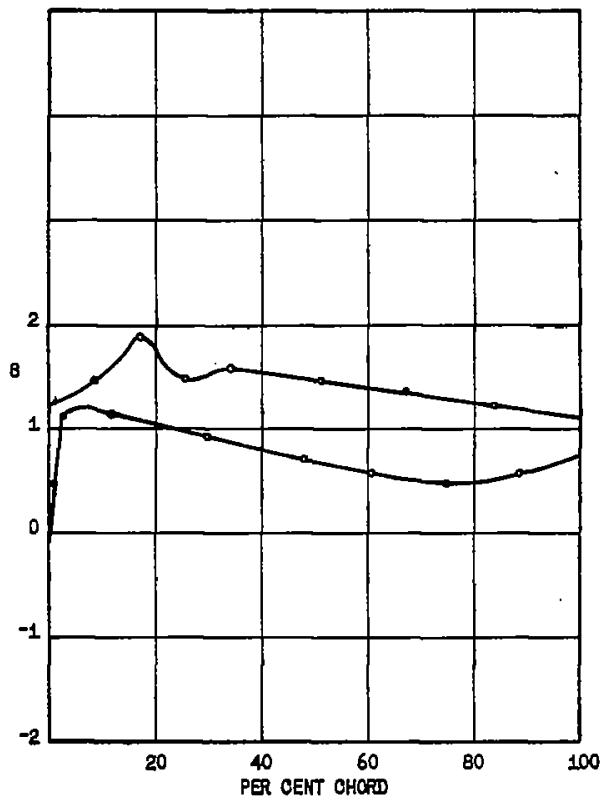

(a) Frame 1.

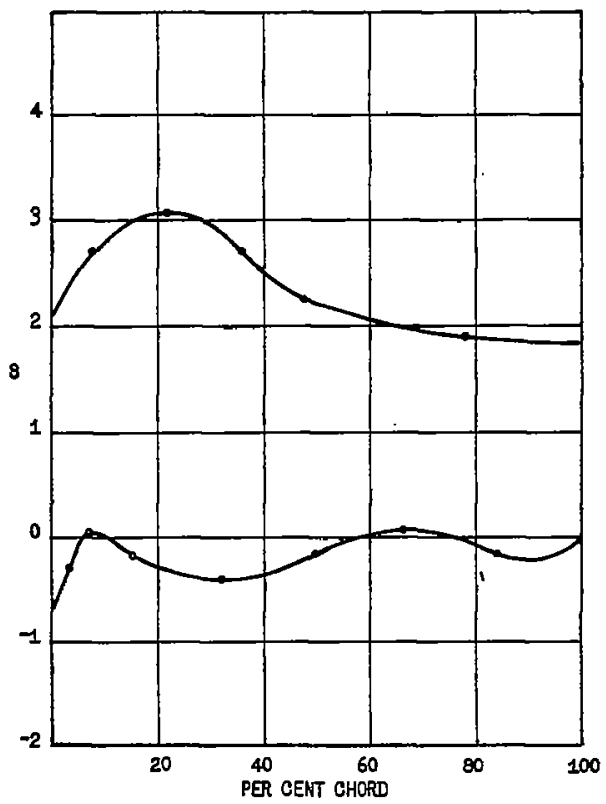

(c) Frame 3 .

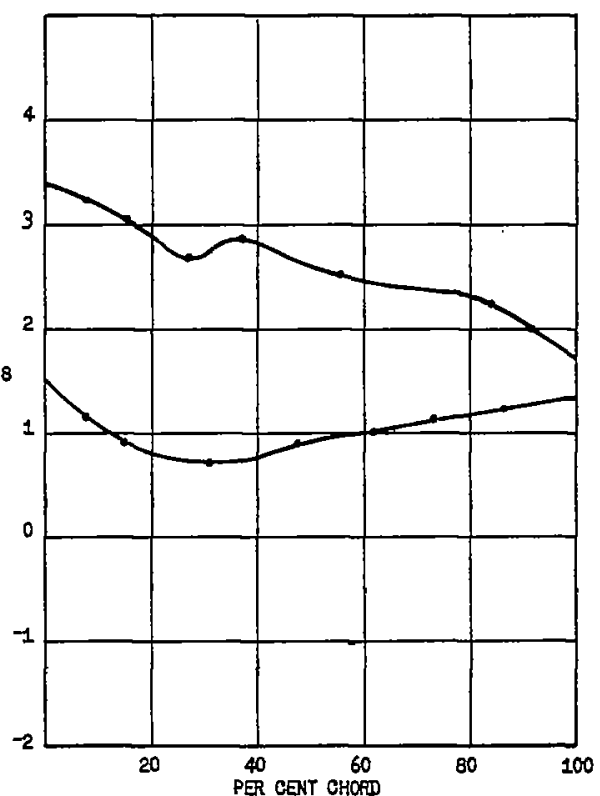

(b) Frame 2.

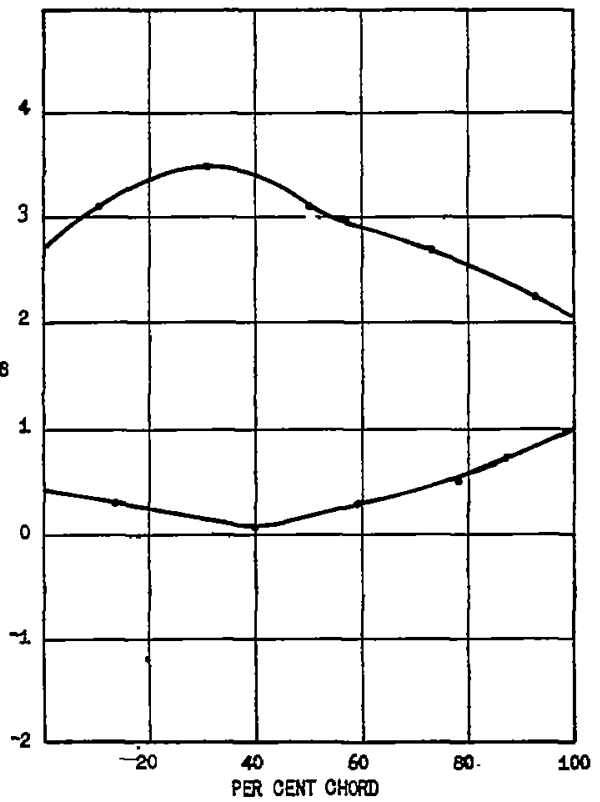

(d) Frame 4.

Flgure 30.- Pressure distributions calculated from figure 2(b). Circles indicate data points obtained from interferogram fringes and by interpolating half fringes. 


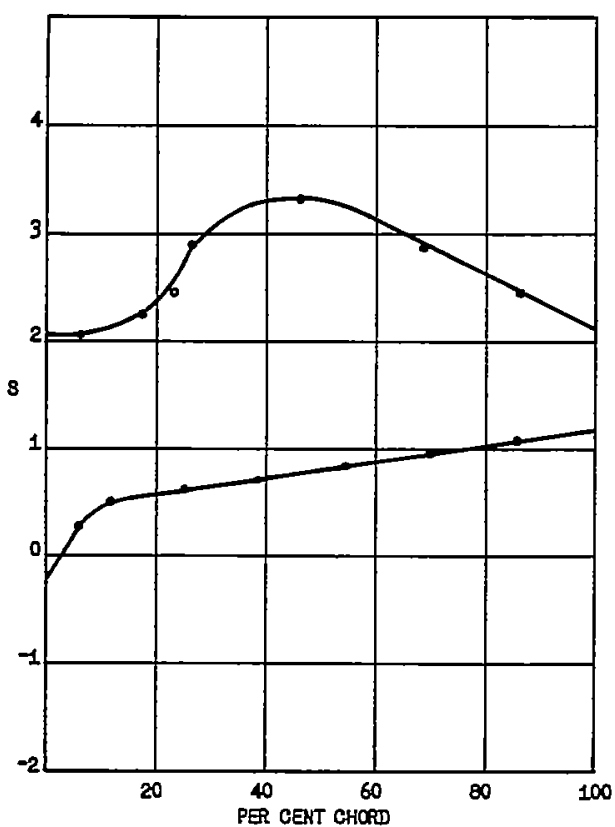

(e) Frame 5 .

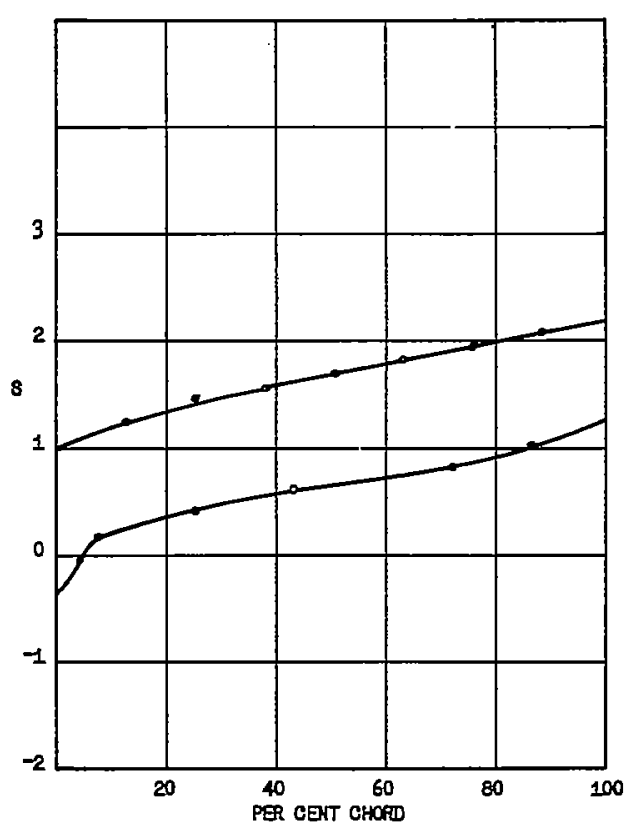

(g) Frame 7 .

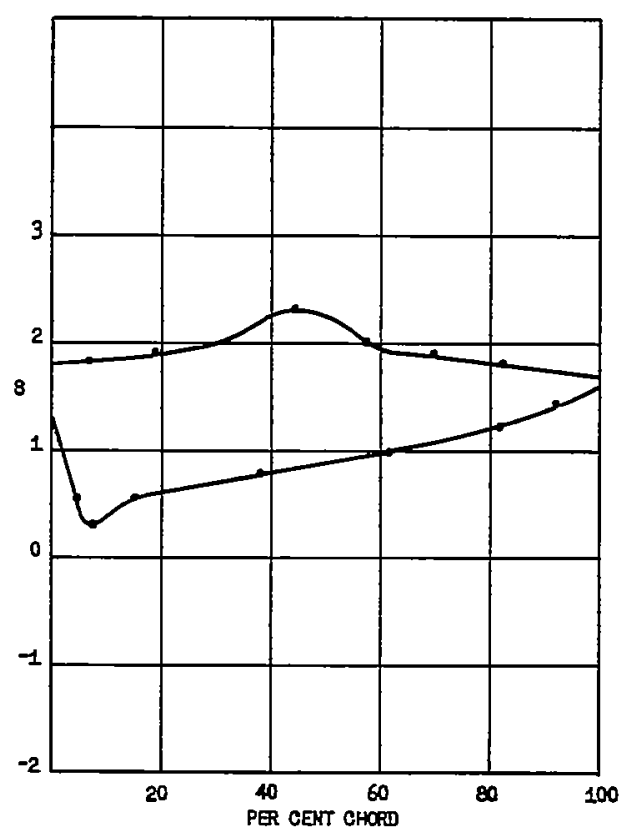

(f) Frame 6.

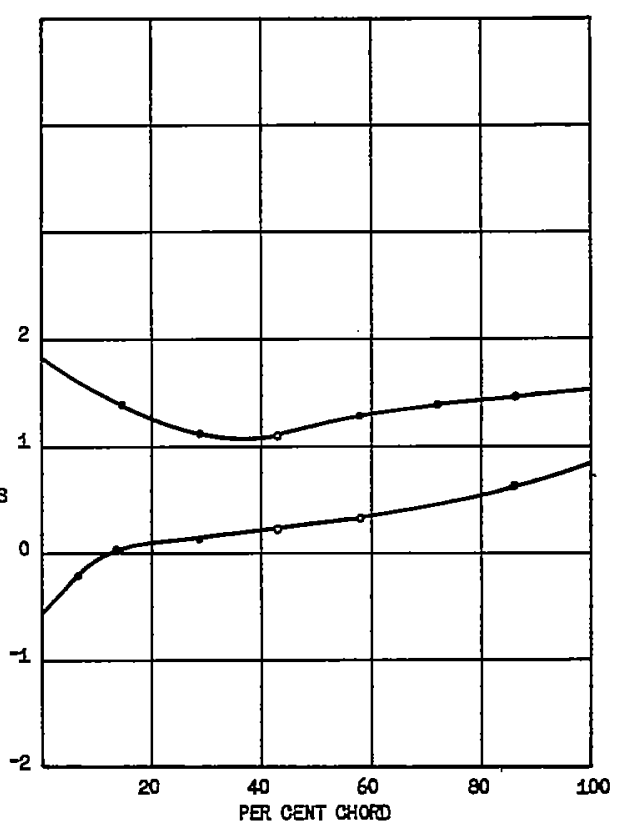

(h) Frame 8.

Figure 30.- Continued. 


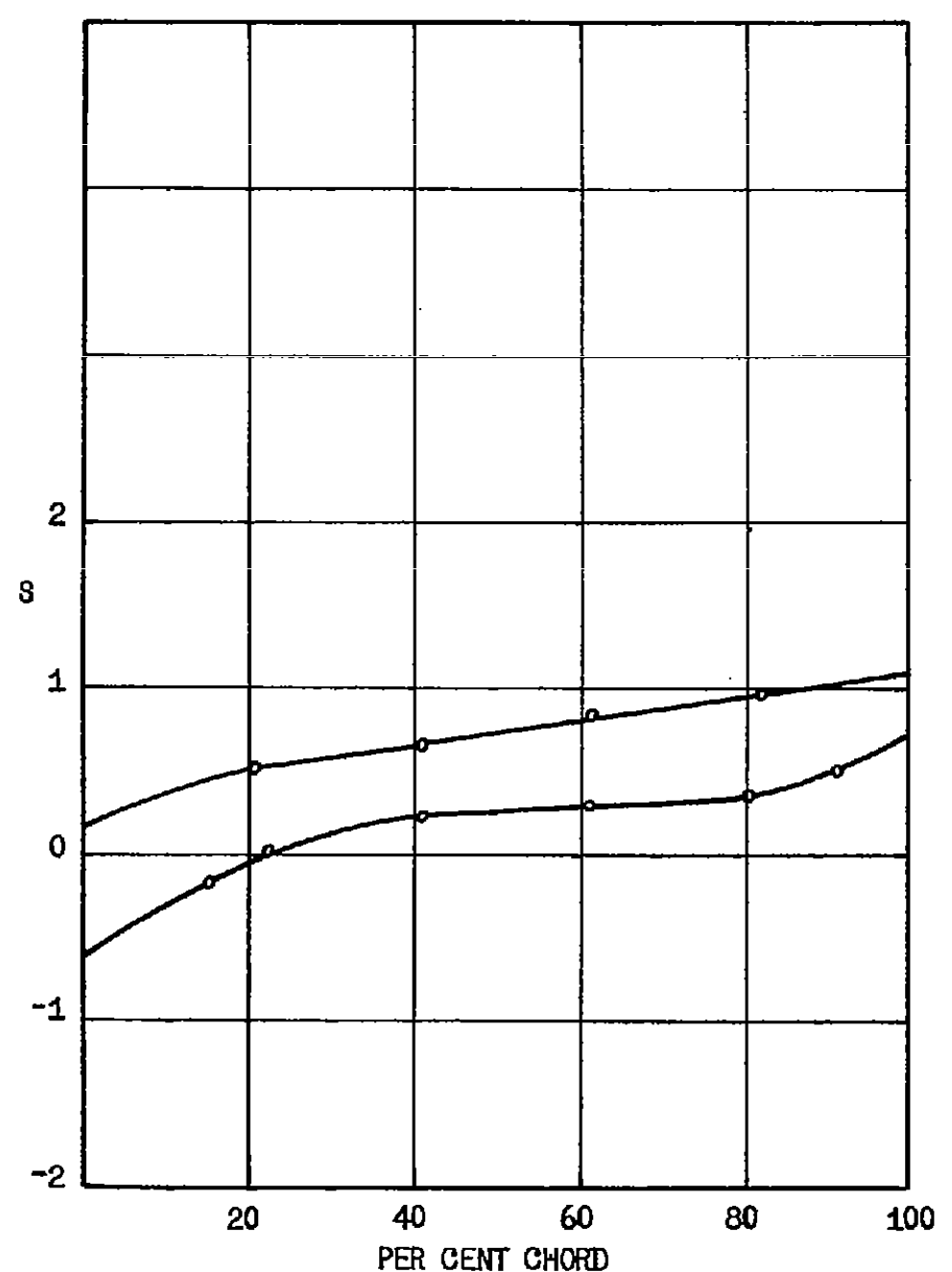

(1) Frame 9.

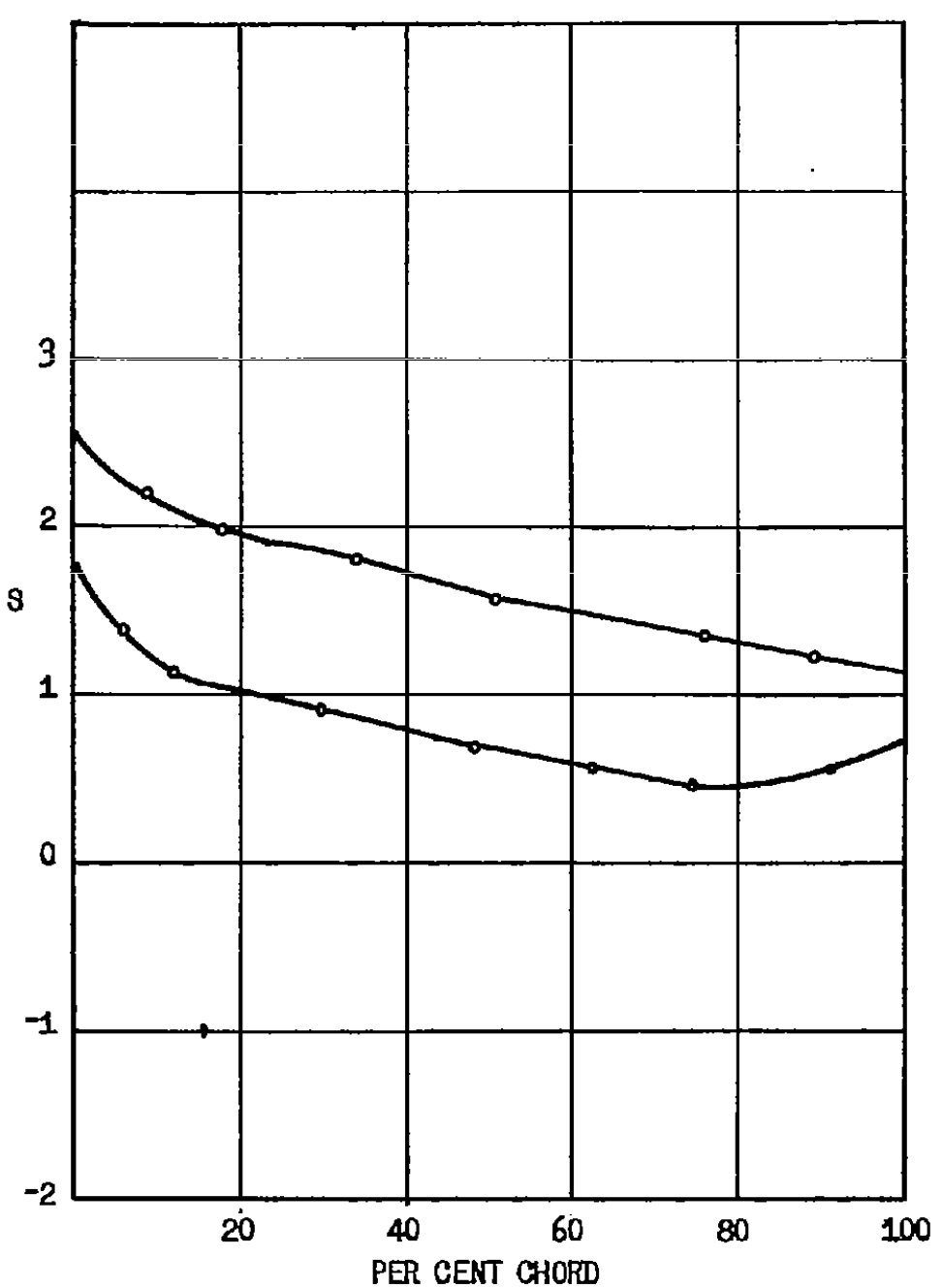

(J) Frame 10.

Flgure 30.- Concluded. 


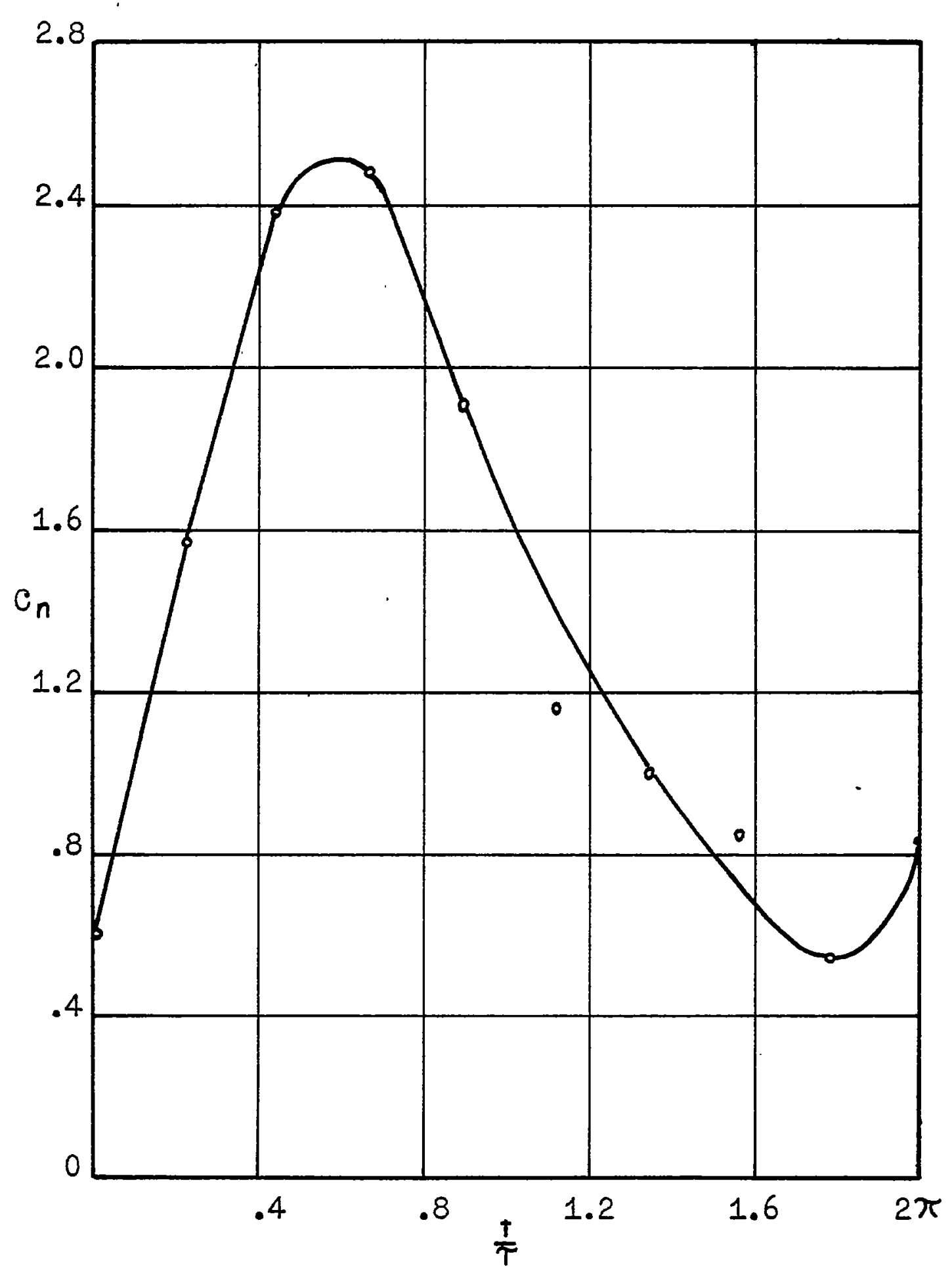

Figure 31.- Normal-force coefficient against time. 


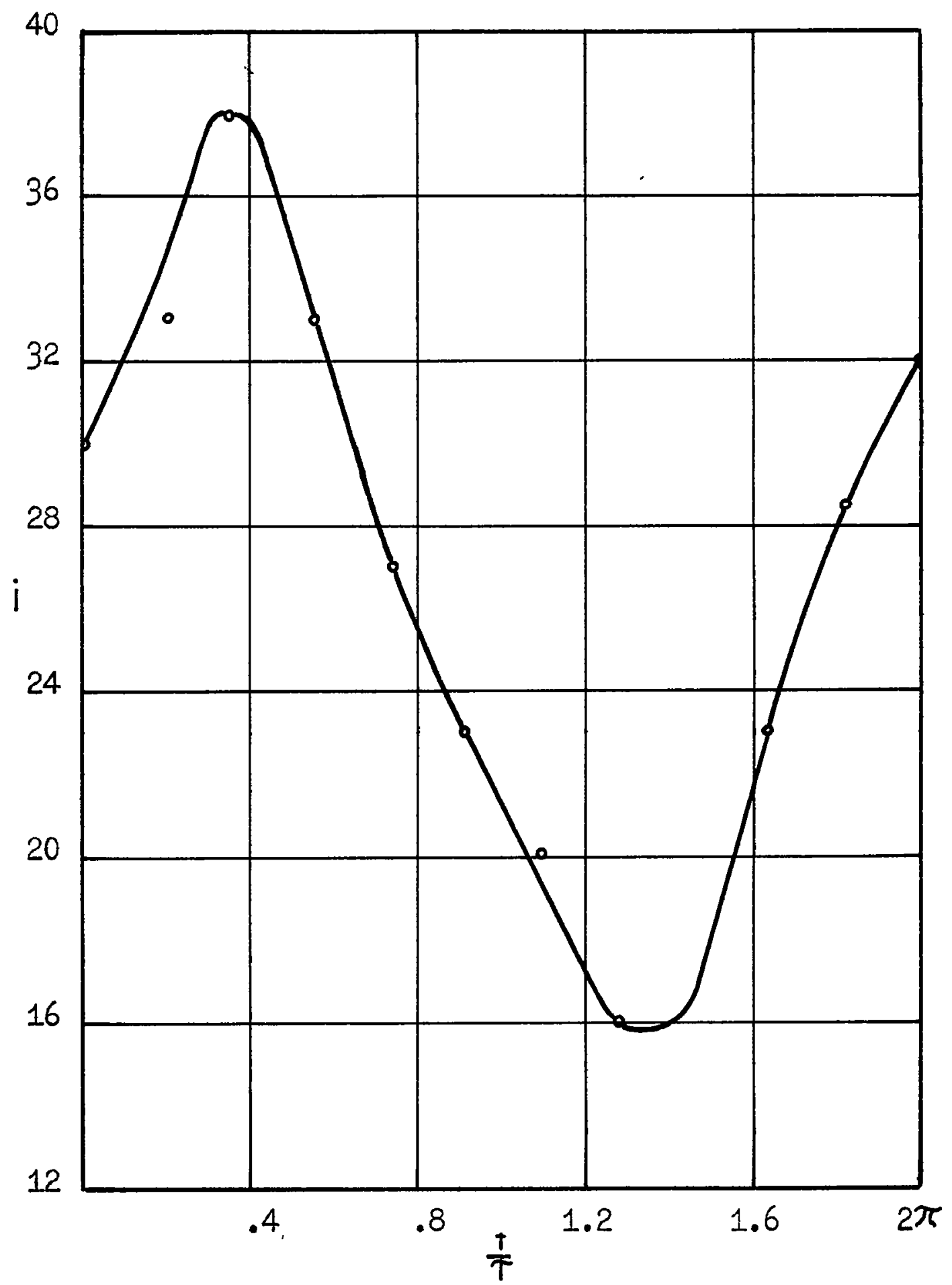

Figure 32.- Local incidence against time. 


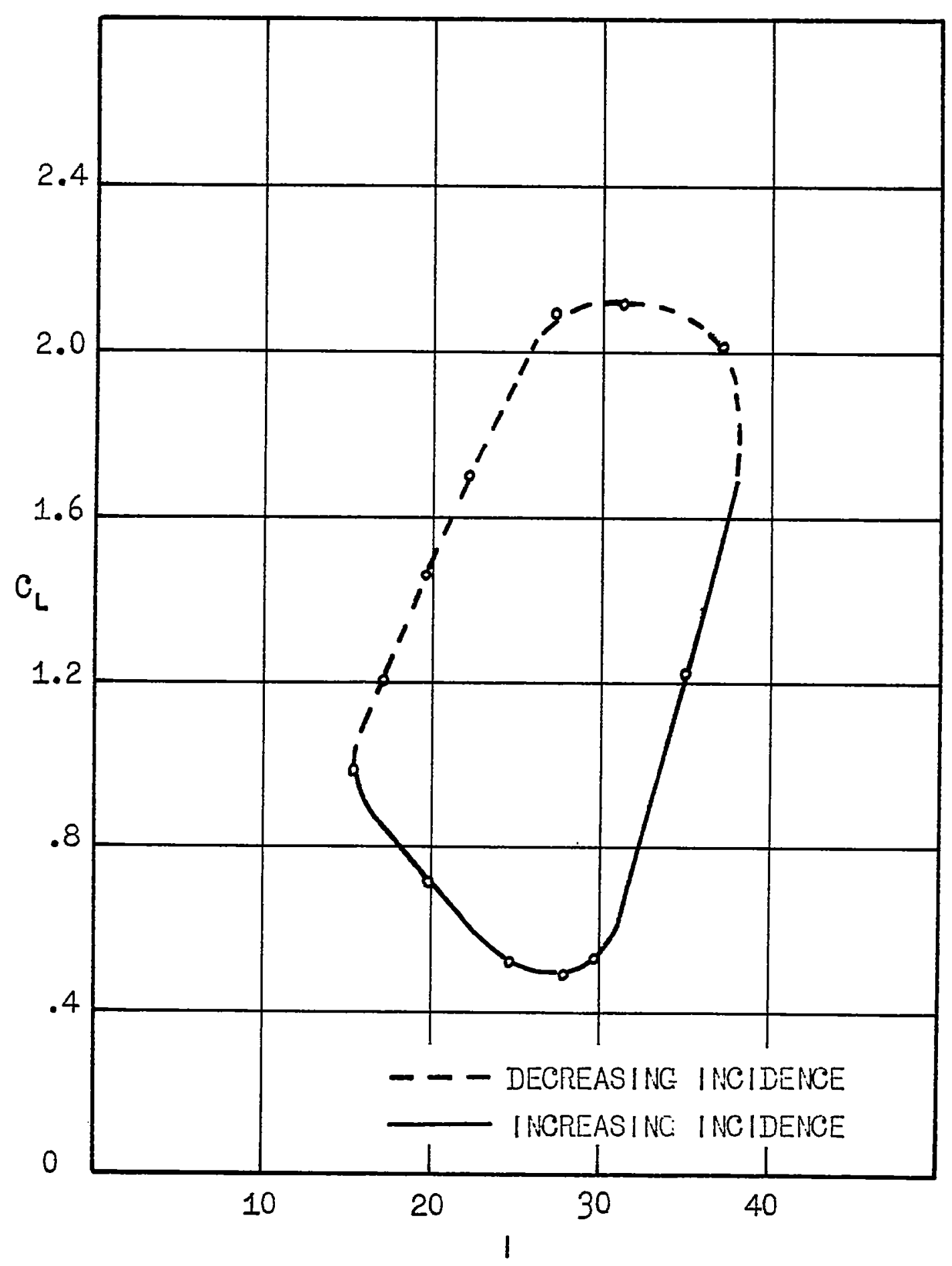

Figure 33.- Lift coefficient against local incidence. 


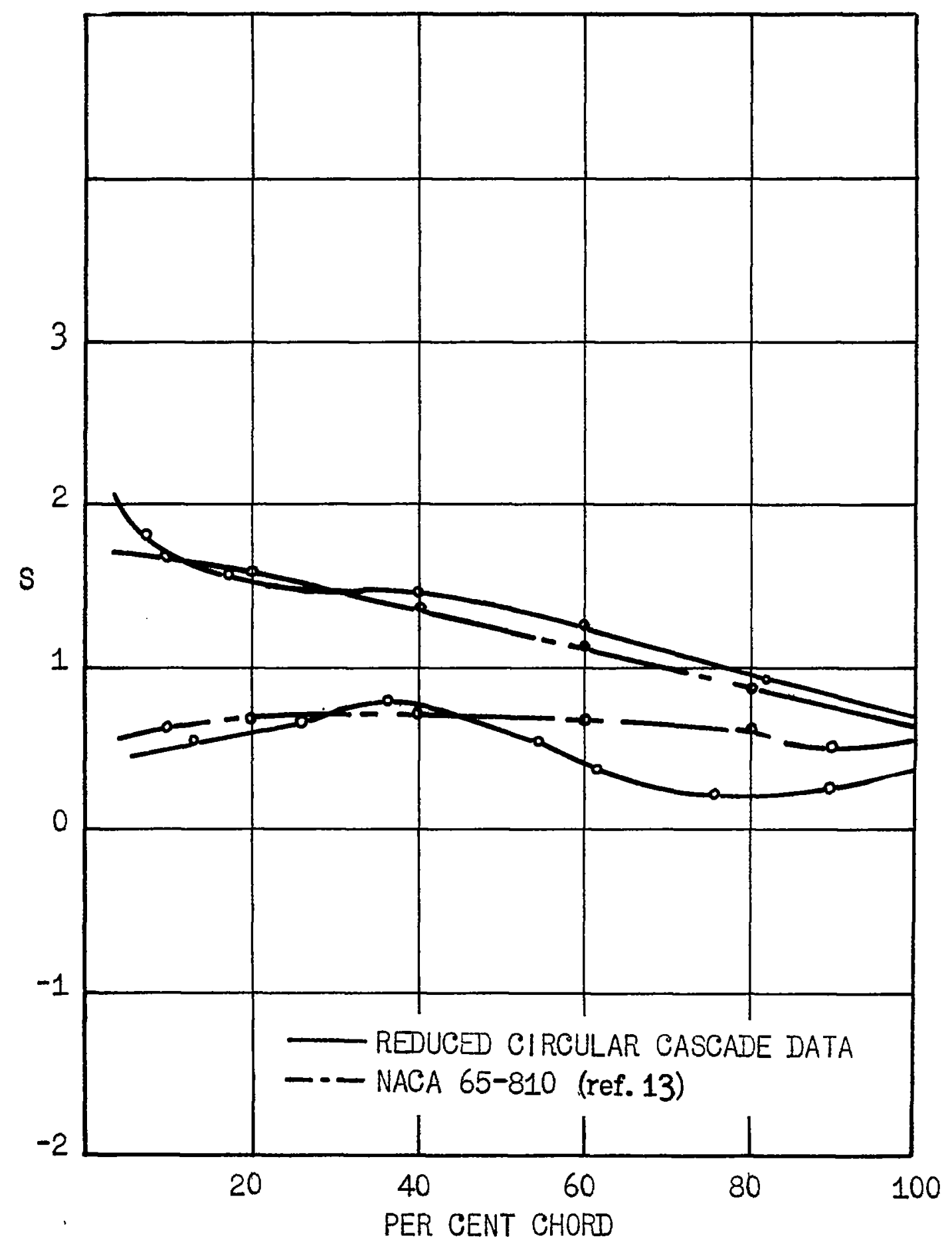

Figure 34.--Comparison of pressure distributions. $\beta_{1}=46.4^{\circ}$. 


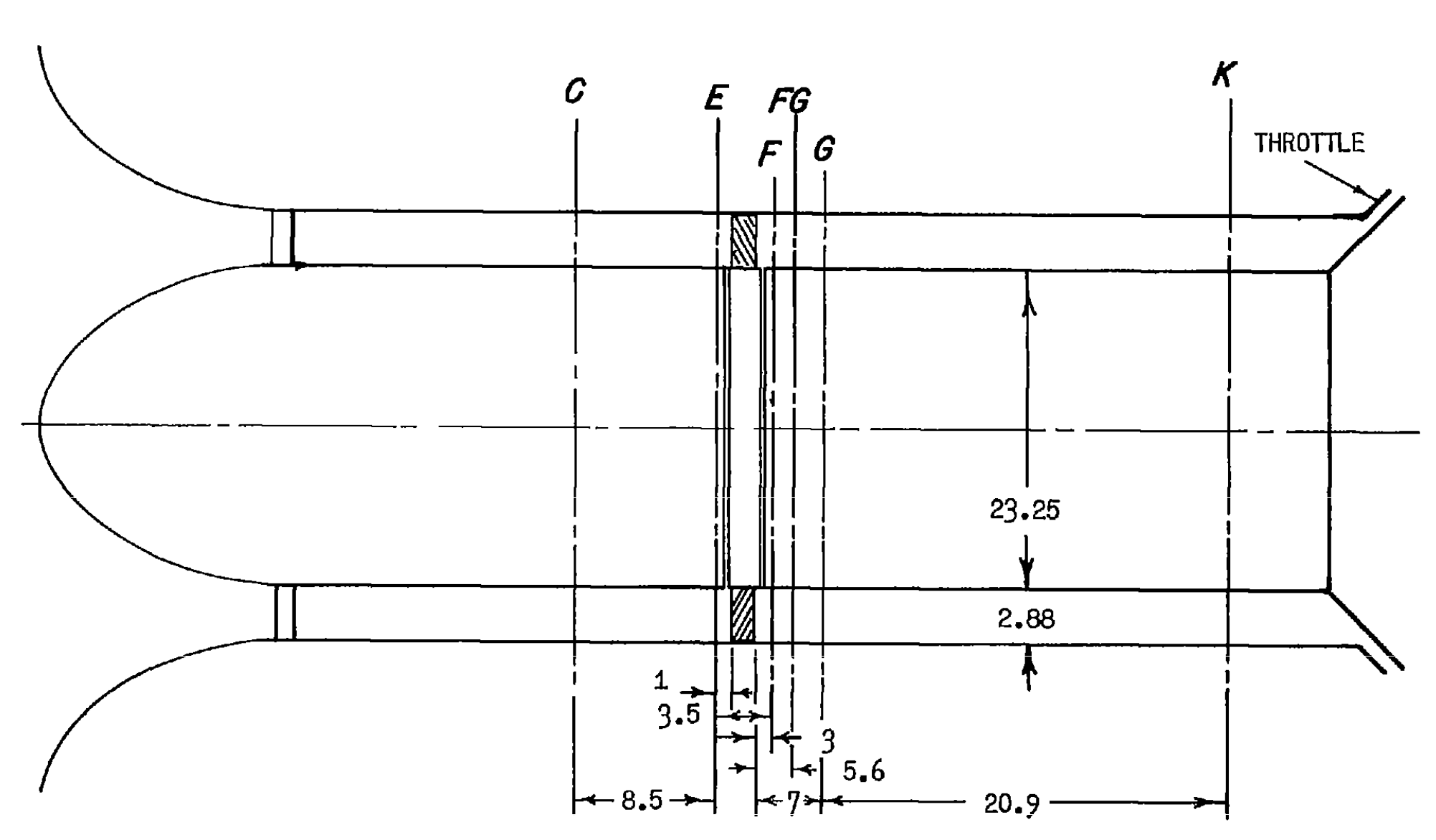

Figure 35.- Schematic drawing of research compressor showing measuring stations. 


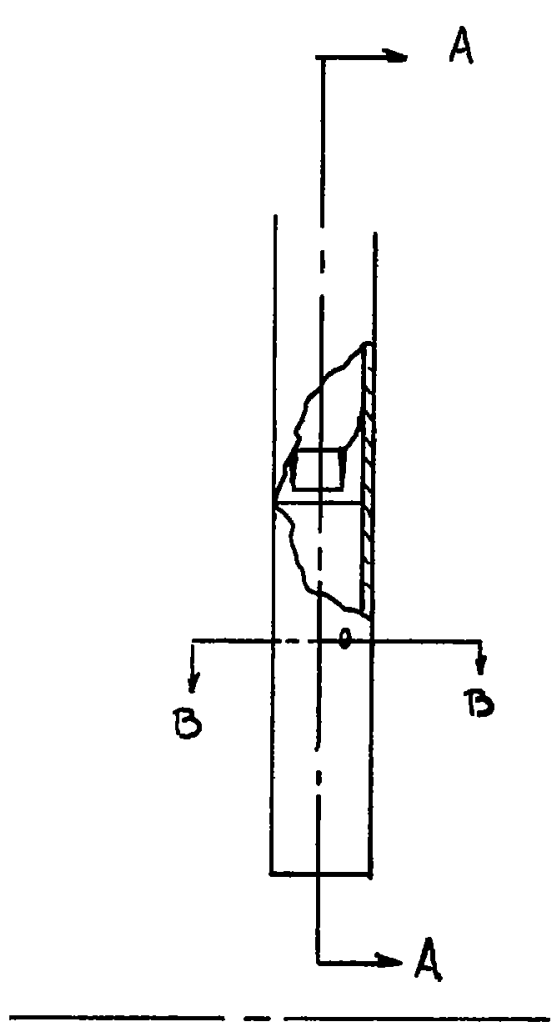

HOT-WIRE PROBE
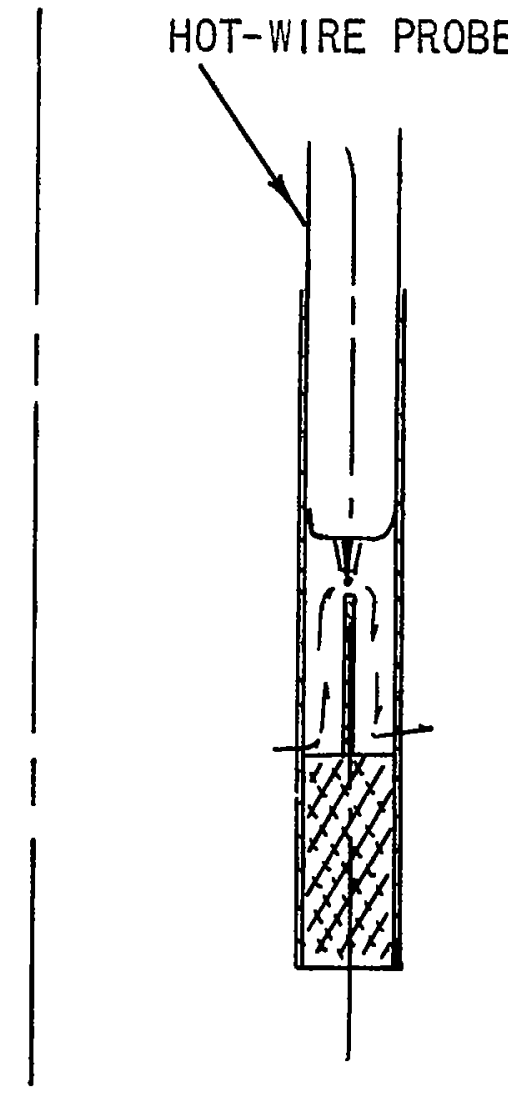

$A-A$

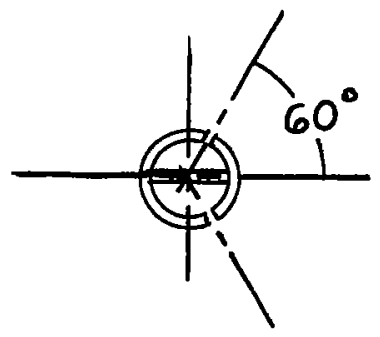

$B-B$

Figure 36.- Hot-wire angle-measuring probe. 


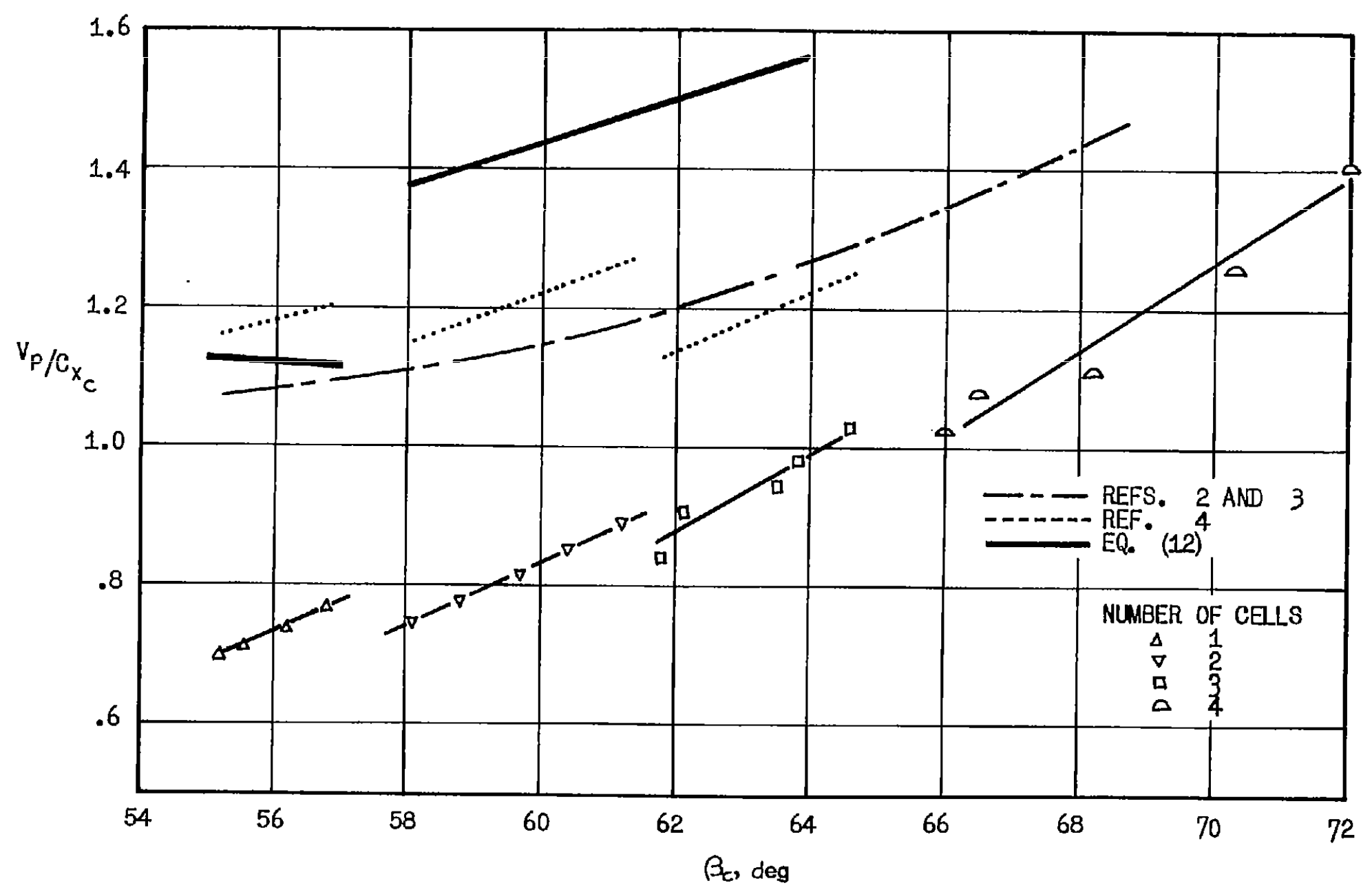

号
是
占
点

Figure 37.- Propagation velocity against $\beta_{C}$. Configuration A. 


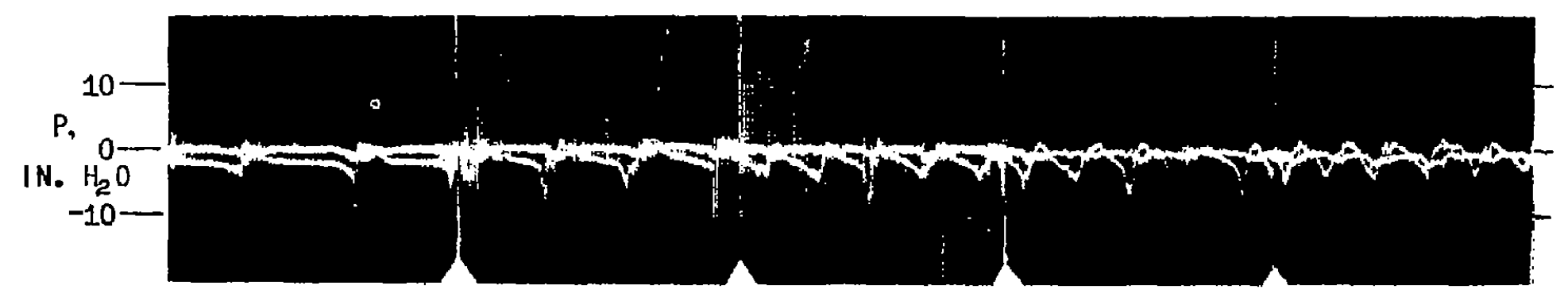
(a) ${ }^{B_{C}}=55.8^{\circ}$;
(b) $\beta_{\mathrm{C}}=59.8^{\circ}$;
(c) $\beta_{\mathrm{C}}=63.1^{\circ}$; two cells.
(d) $\beta_{C}=66.6^{\circ}$; three cells.
(e) $\beta_{\mathrm{C}}=70.3^{\circ}$; four cells. four cell.s.

F1gure 38. - Static pressure at outer wall. Configuration A; upper trace at station FG; lower trace at station $E$; time from left to right. 


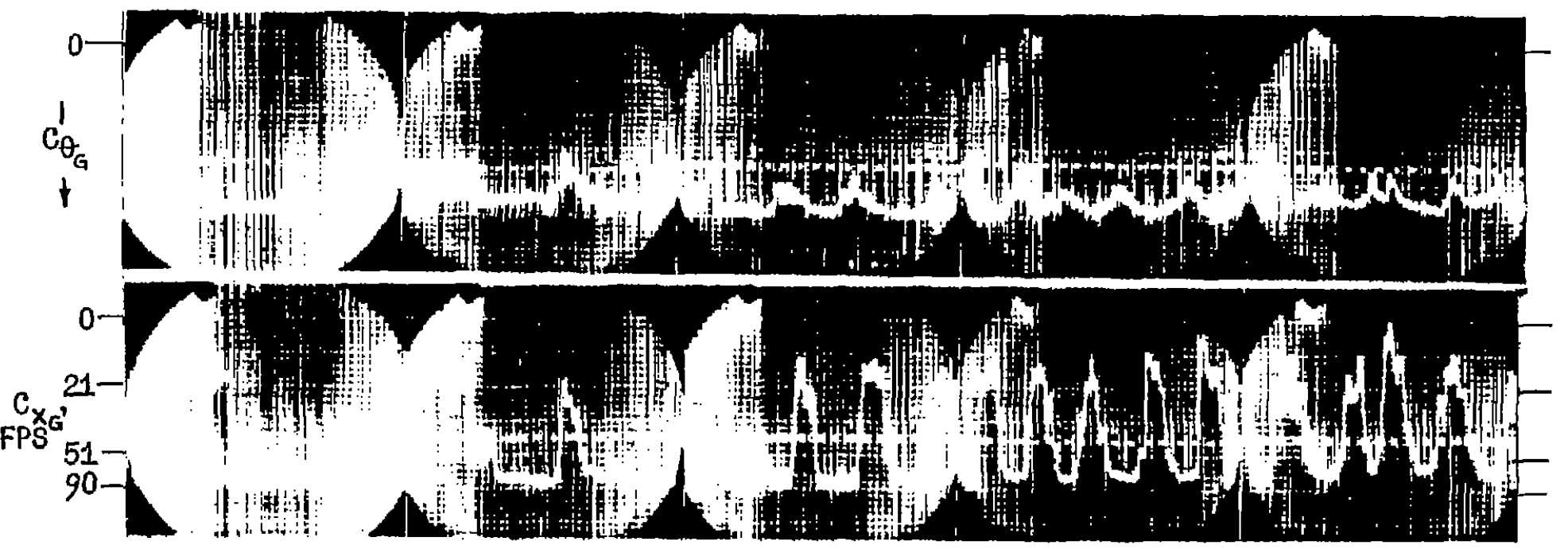
(a) $\beta_{\mathrm{C}}=56.7^{\circ}$
(b) $\beta_{C}=60.1^{\circ}$;
(c) $\beta_{C}=63.4^{\circ}$;
(d) $\beta_{\mathrm{C}}=67.3^{\circ}$; three cells. four cells.
(e) $\beta_{C}=71.0^{\circ}$ four cells.

Figure 39.- Hot-wire traces of $C_{x_{G}}$ and $C_{\theta_{G}}$. Configuration $A$. 


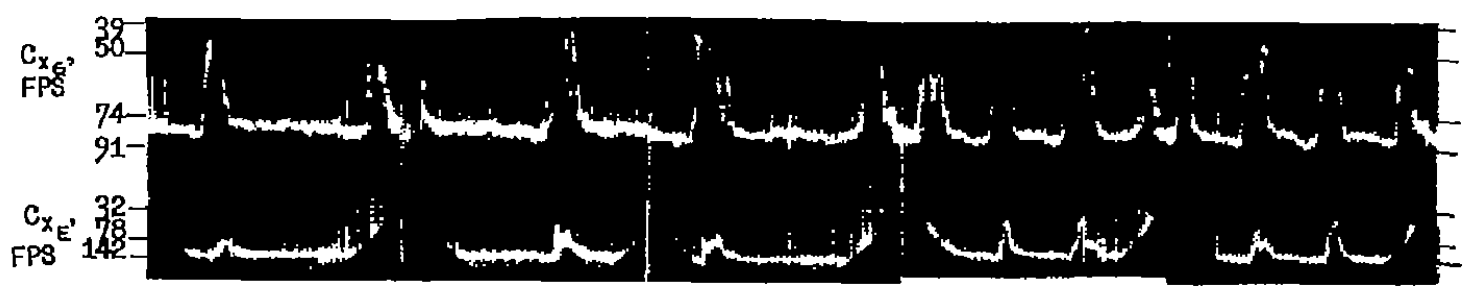

(a) $\beta_{C}=55.60^{\circ}$; one cell. (b) $\beta_{C}=56.1^{\circ}$; one cell. (c) $\beta_{\mathrm{C}}=57.0^{\circ}$; one cell. (d) $\beta_{C}=57.7^{\circ}$; two cells. (e) $\beta_{C}=58.3^{\circ}$; two cells.

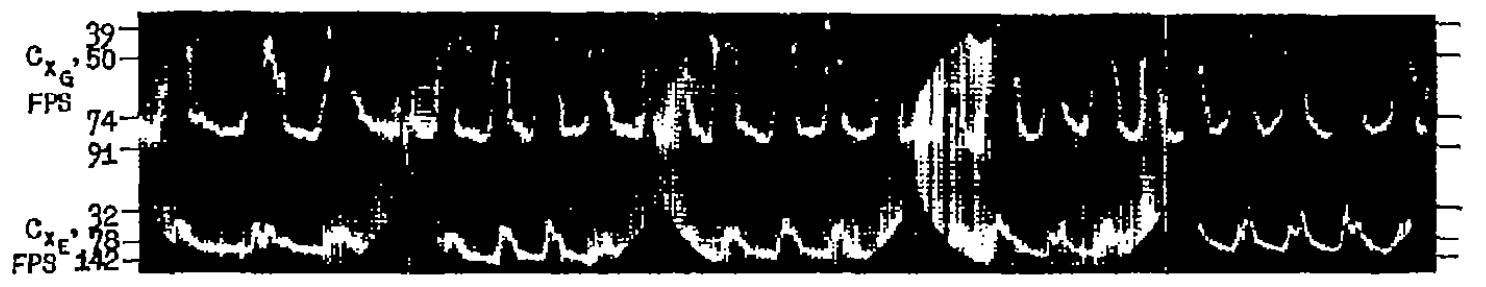

(f) $\beta_{C}=61.1^{\circ}$;

(g) $\beta_{C}=61.5^{\circ}$;

(h) $\beta_{\mathrm{C}}=63.1^{\circ}$; three cells.

(1) $\beta_{\mathrm{C}}=64.5^{\circ}$; three cells.

(j) $\beta_{C}=65.6^{\circ}$; two cellis.

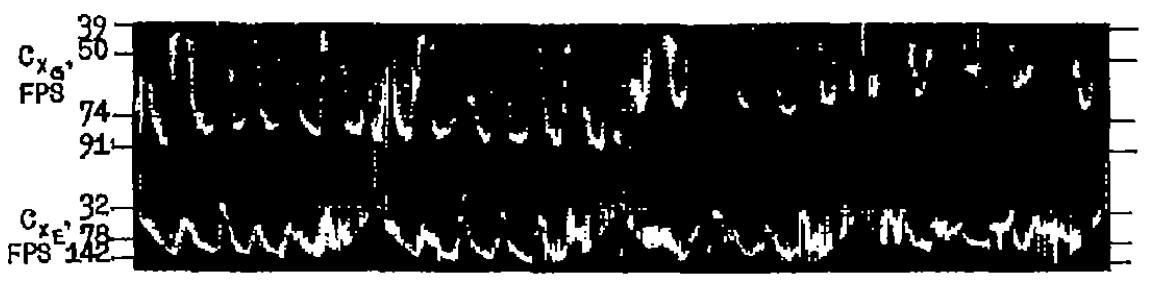
(k) ${ }^{\beta} \mathrm{C}=65.9^{\circ}$;
(2) $\mathrm{B}_{\mathrm{C}}=67.3^{\circ}$;
(m) $\beta_{\mathrm{C}}=70.7^{\circ}$; four cells. four cells. four cells.
(n) $\beta_{C}=74.6^{\circ}$; four cells.

F1gure 40.- Hot-wire traces of $\mathrm{C}_{\mathrm{X}_{G}}$ and $\mathrm{C}_{\mathrm{X}_{\mathrm{E}}}$. Configuration $\mathrm{A}$. 


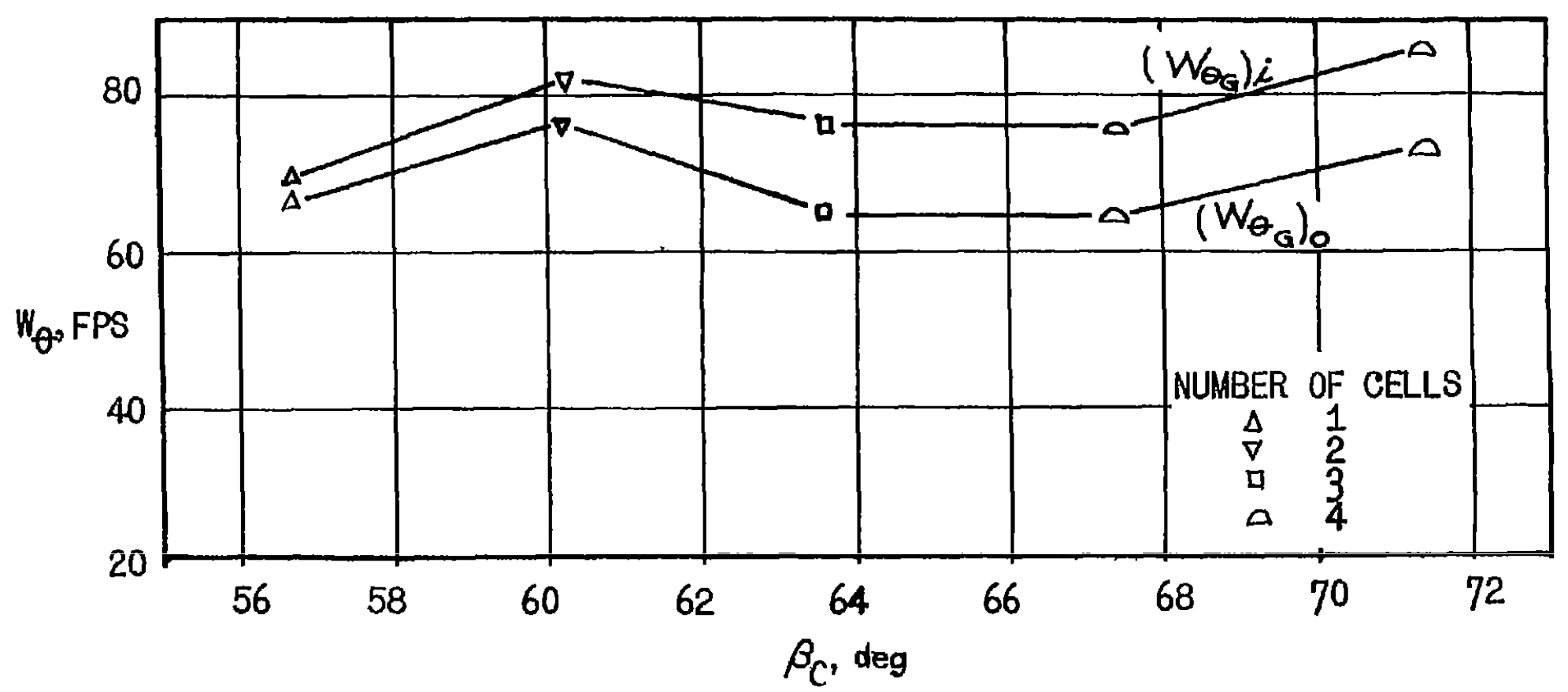

Figure 41.- Velocity components versus $\beta_{C}$. Configuration A. 


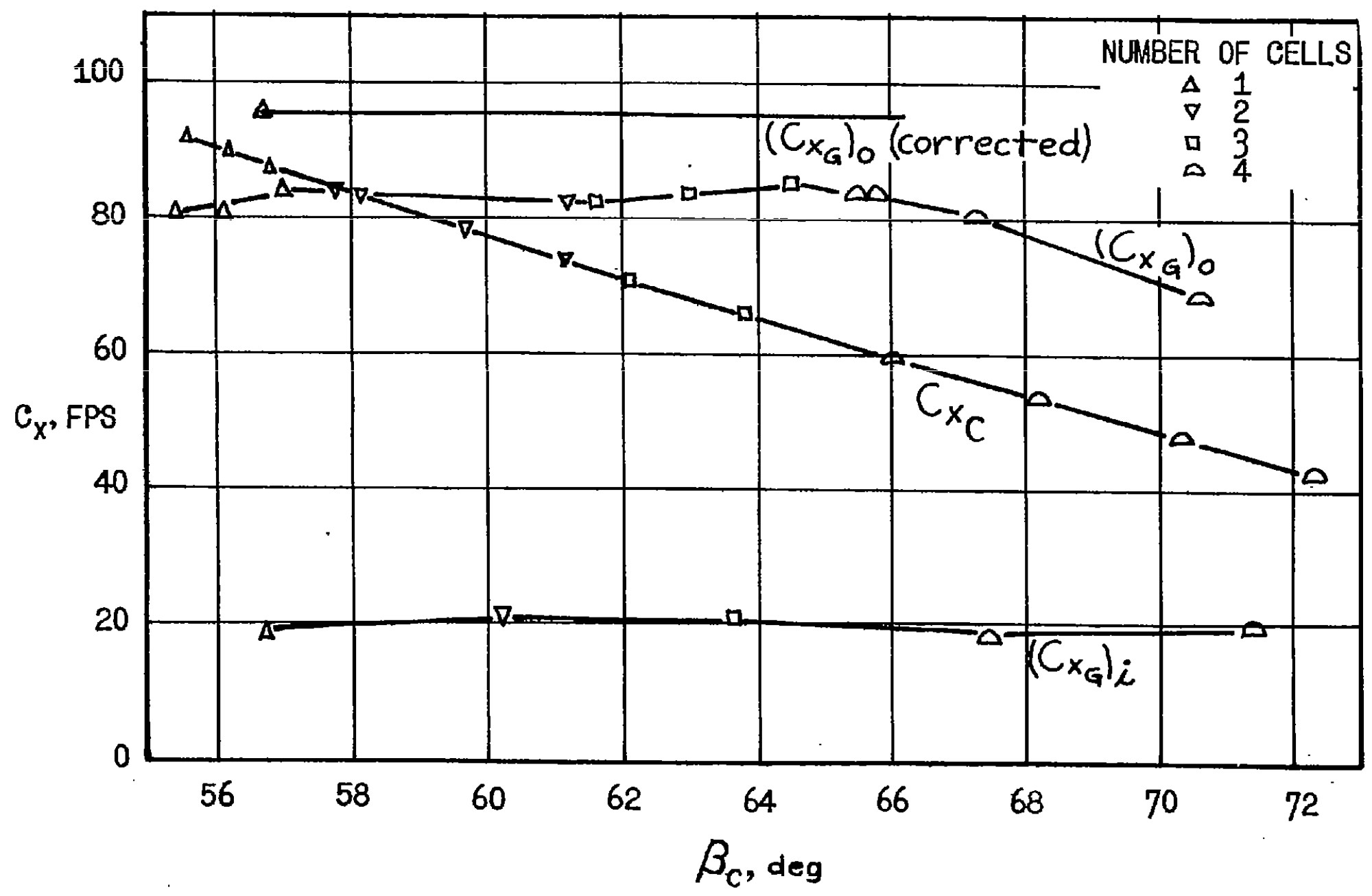

Figure 42.- Veloc1ty components versus $\beta_{C}$. Configuration A. 


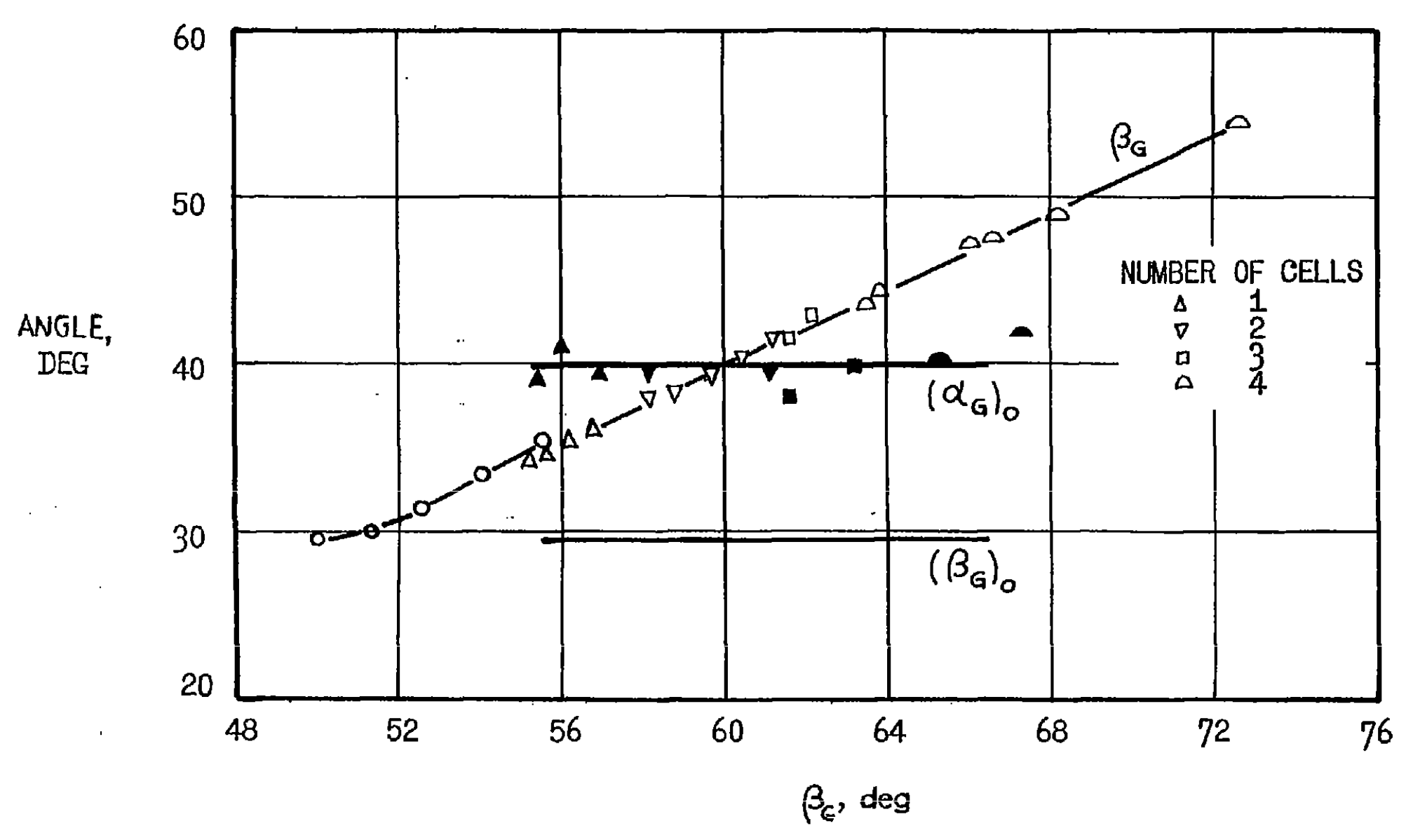

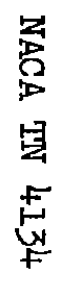

F1gure 43. - Flow angles versus $\beta_{C}$. Configuration A. 


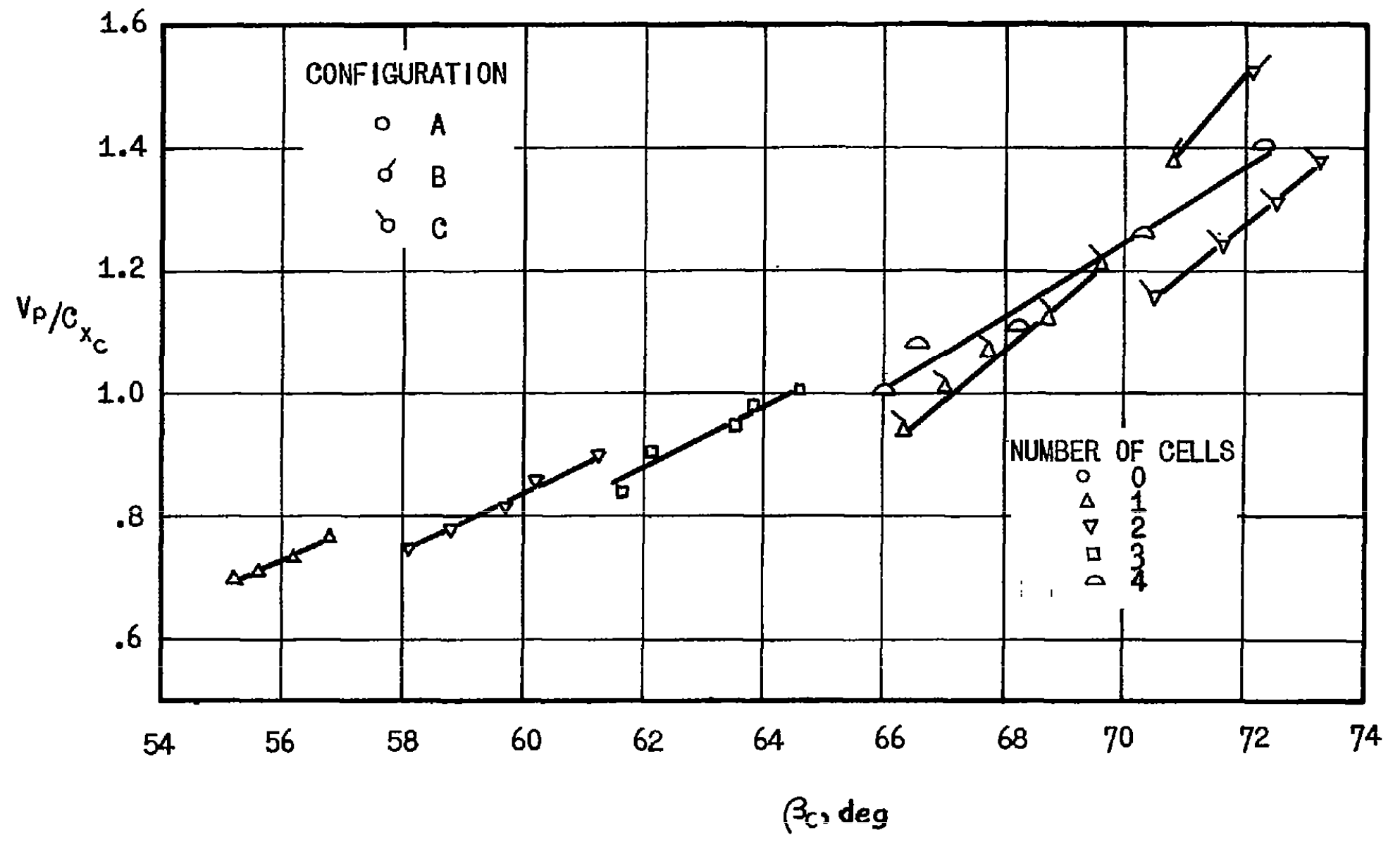

F1gure 44.- Plot of propagation velocity versus ${ }^{\beta}$. 


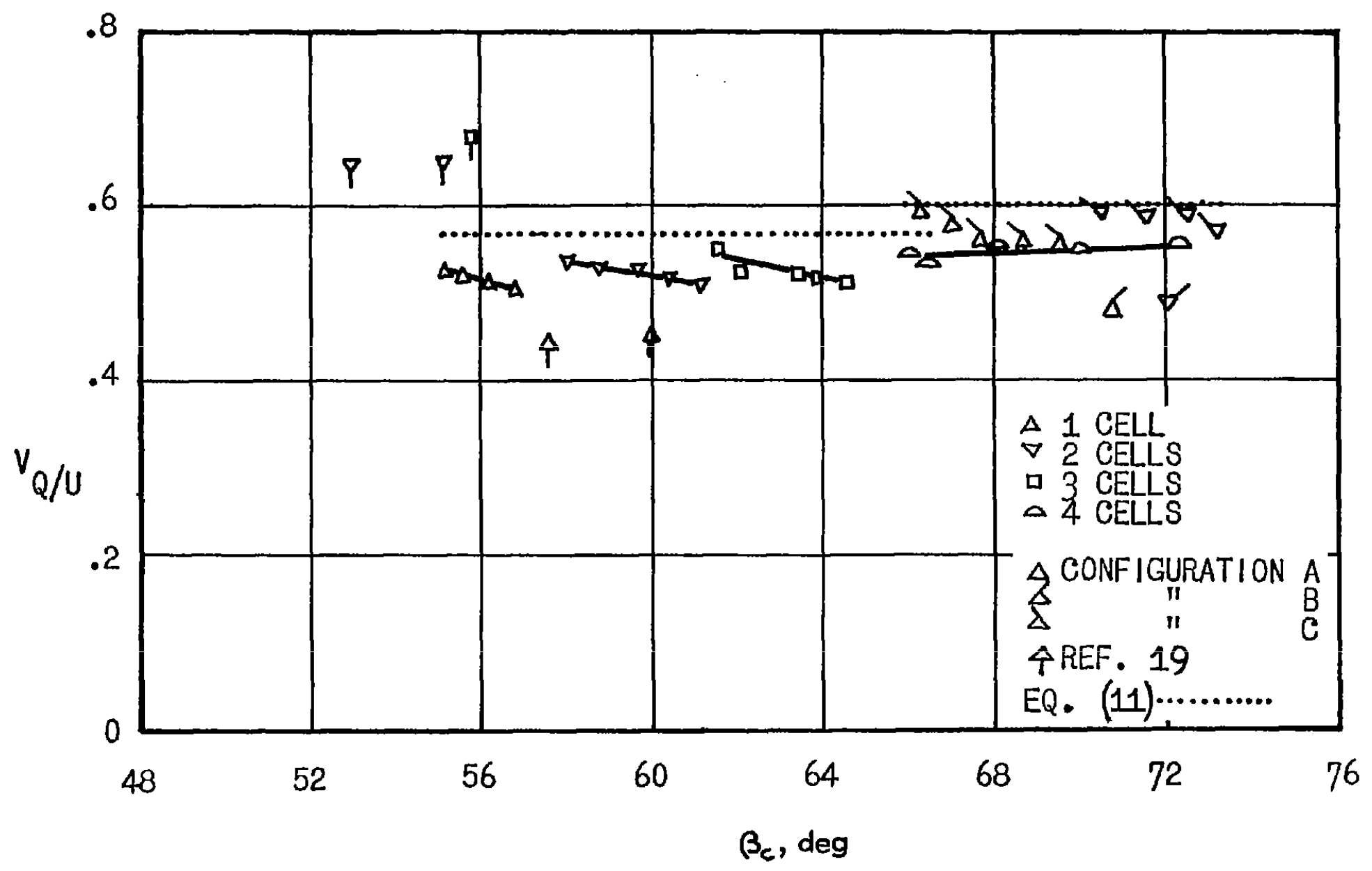

Flgure 45. - Plot of propagation velocity versus $\beta_{C}$. 


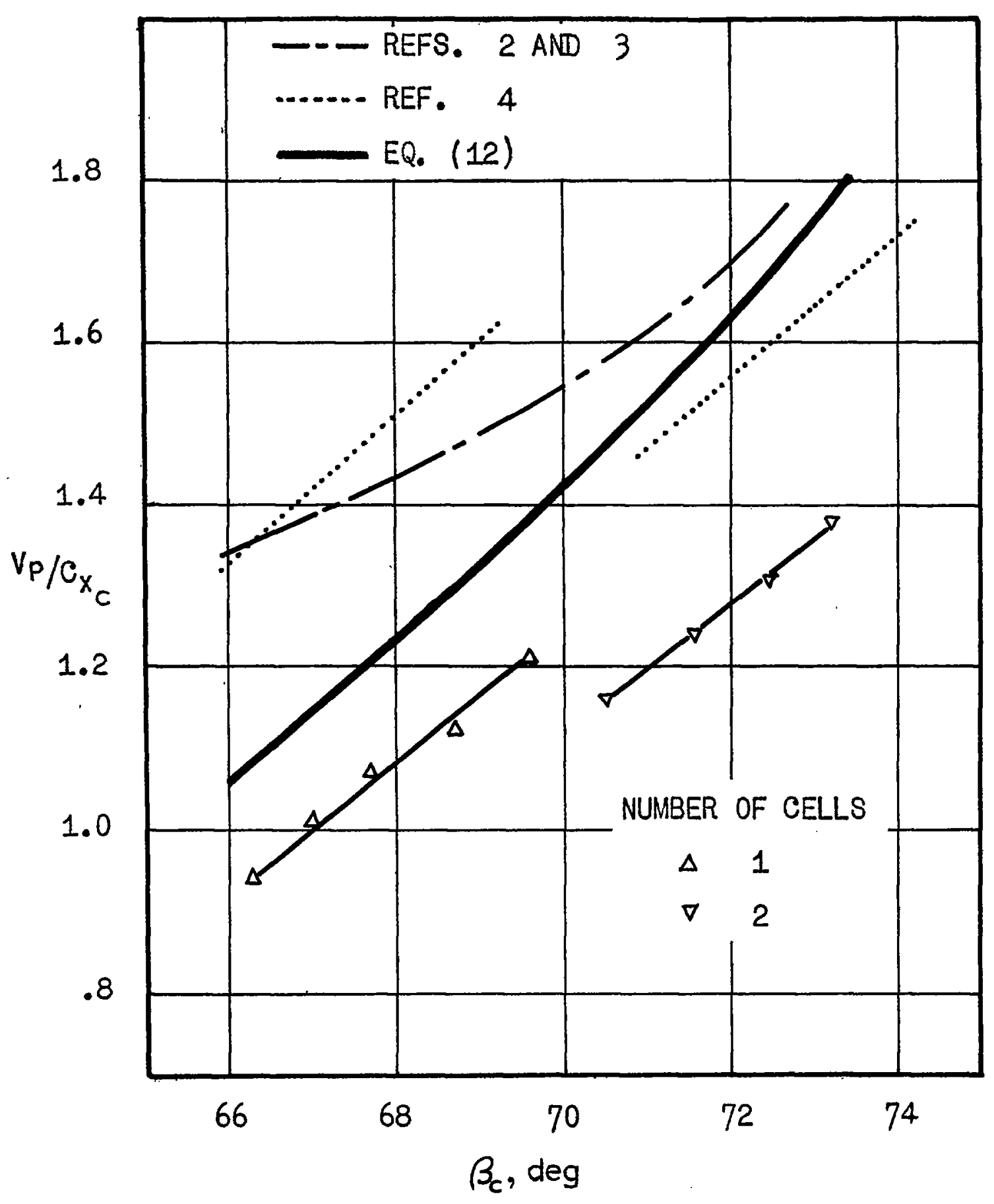

F1gure 46.- Propagation veloc1ty versus $\beta_{C}$. Configuration C. 


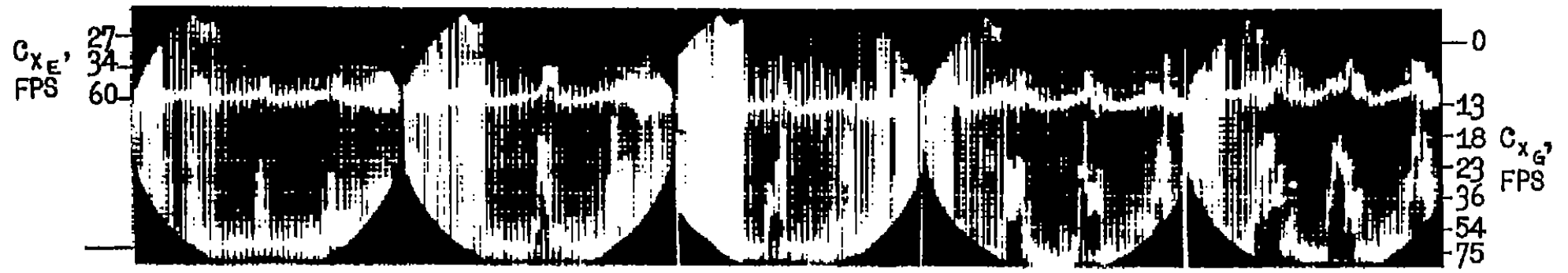
(a) $\beta_{C}=66.2^{\circ}$; one cell.
(b) $\beta_{C}=66.6^{\circ}$;
(c) $\beta_{\mathrm{C}}=67.0^{\circ}$; one cell.
(d) $\beta_{C}=68.0^{\circ}$; one cell.
(e) $\beta_{C}=69.2^{\circ}$; one cell.

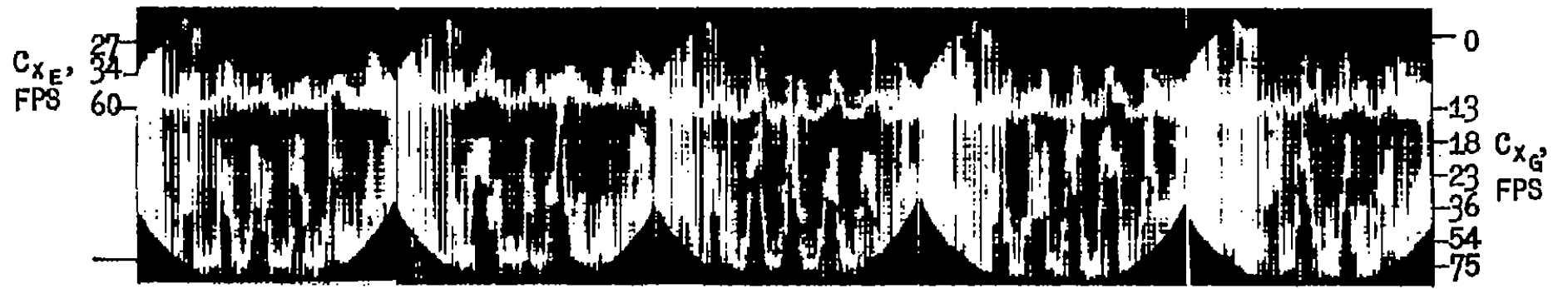

(f) $\beta_{C}=70.0^{\circ}$;
two cell.s.

(g) $\beta_{\mathrm{C}}=71.0^{\circ}$;

(h) $\beta_{\mathrm{C}}=71.8^{\circ}$;
two cells.

(1) $\beta_{\mathrm{C}}=73.0^{\circ}$;

(j) $\beta_{C}=74.6^{\circ}$;

F1gure 47.- Hot-wire traces of $C_{x_{E}}$ and $C_{x_{G}}$. Configuration C. 


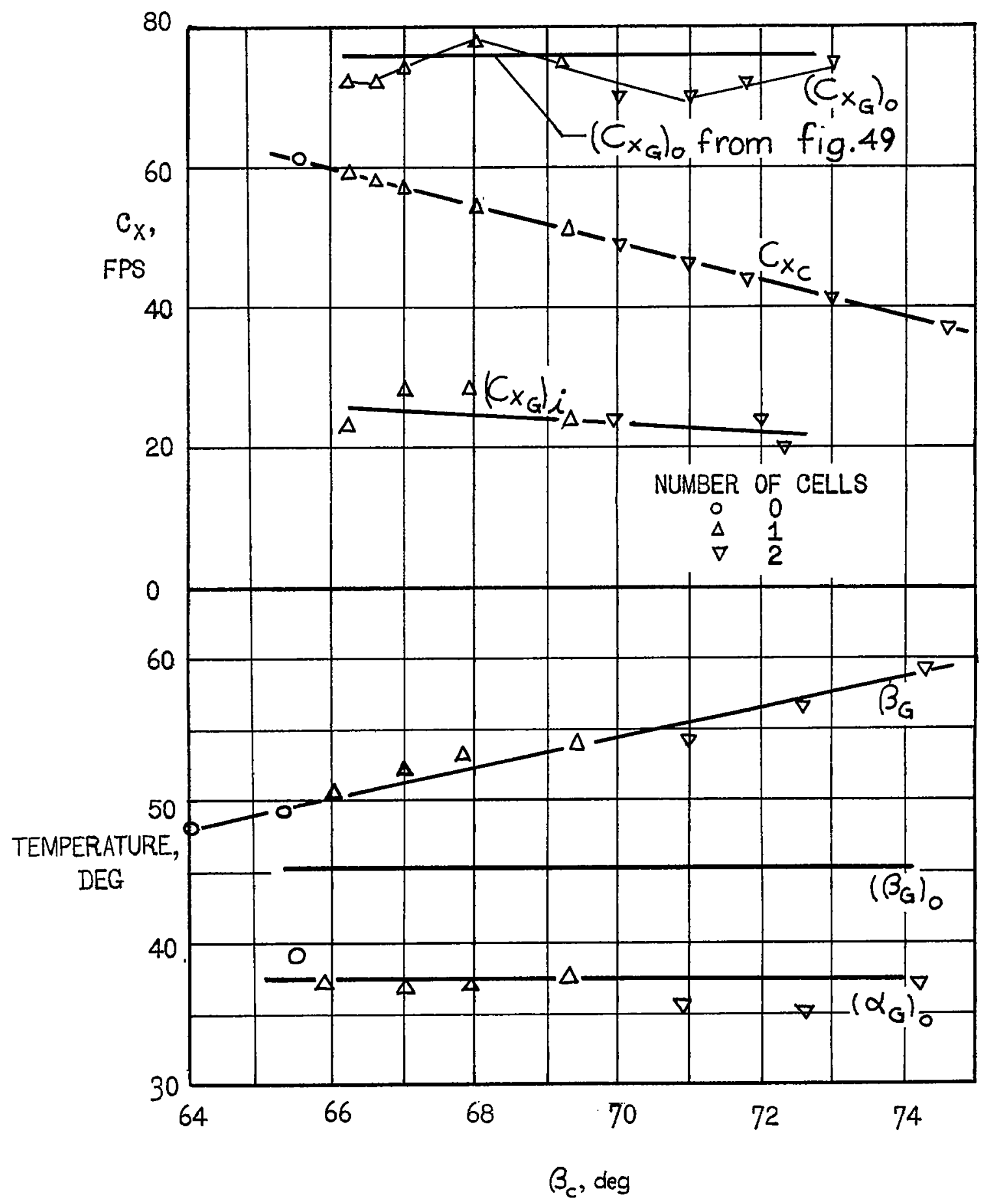

F1gure 48.- Velocity components and flow angles versus $\beta_{C}$. Configuration $\mathrm{C}$. 

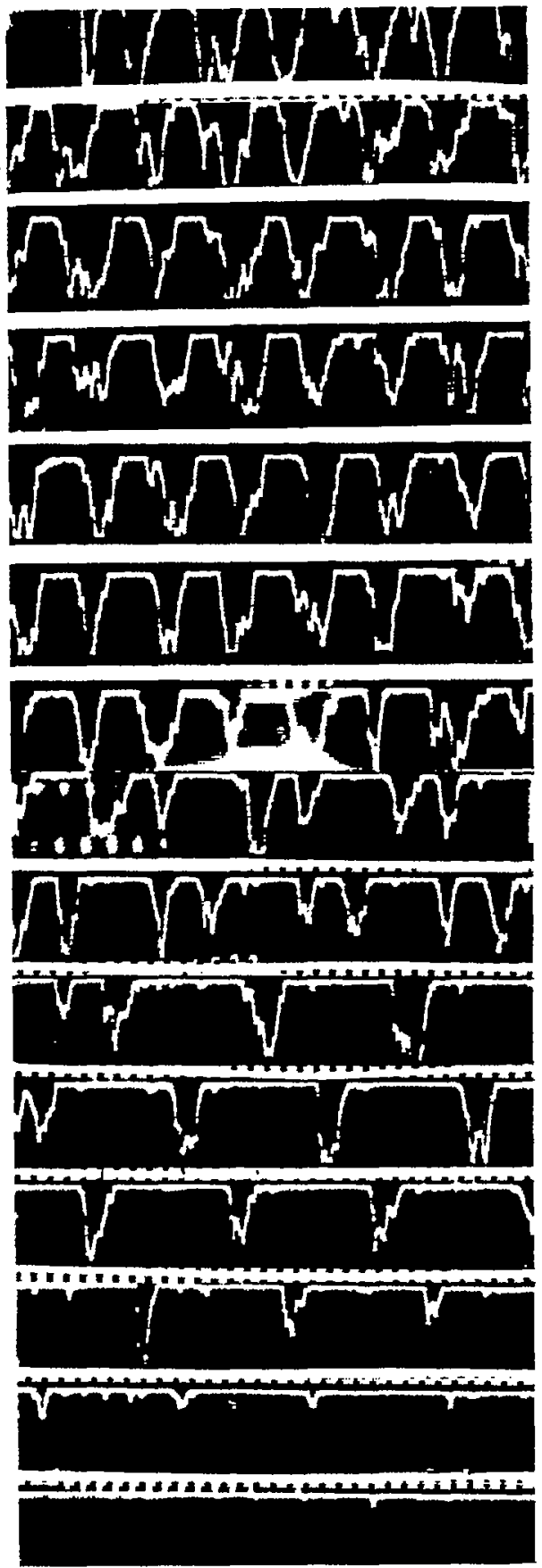

FIgure 49. - Strip-film record of hot-wire trace showing $C_{X_{G}}$ as throttle is opened. Configuration $\mathrm{C}$; time from left to right; $\mathrm{C} \uparrow$. 


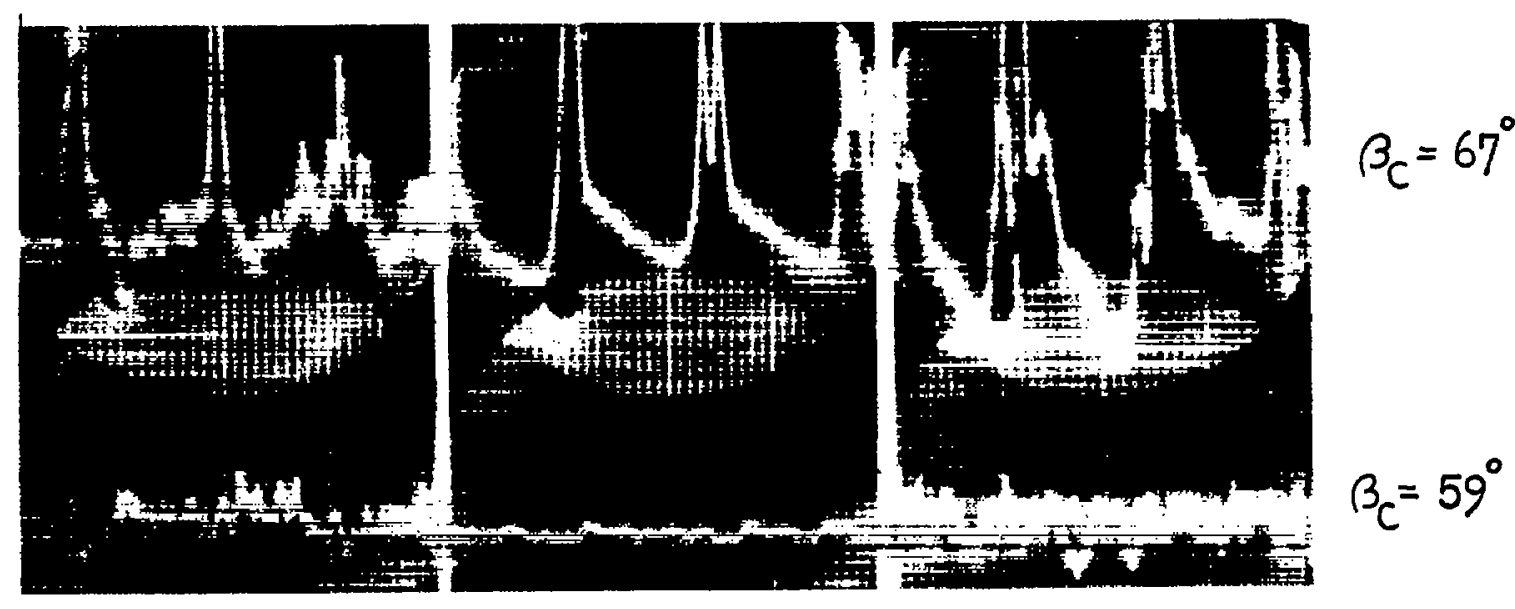
(a) Hub.
(b) Mean radius.
(c) TIp.

Figure 50.- Hot-wire traces at station F. configuration C. 
NACA TN 4134

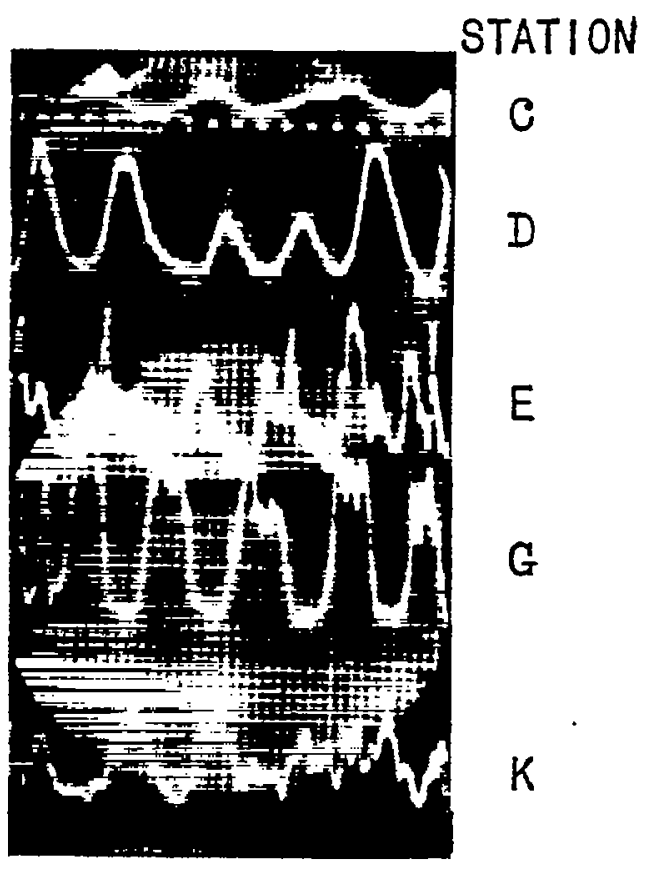

F1gure 51.- Hot-wire traces of $C_{X}$. Configuration A. $C_{X} \downarrow ; \beta_{C}=70^{\circ}$. 


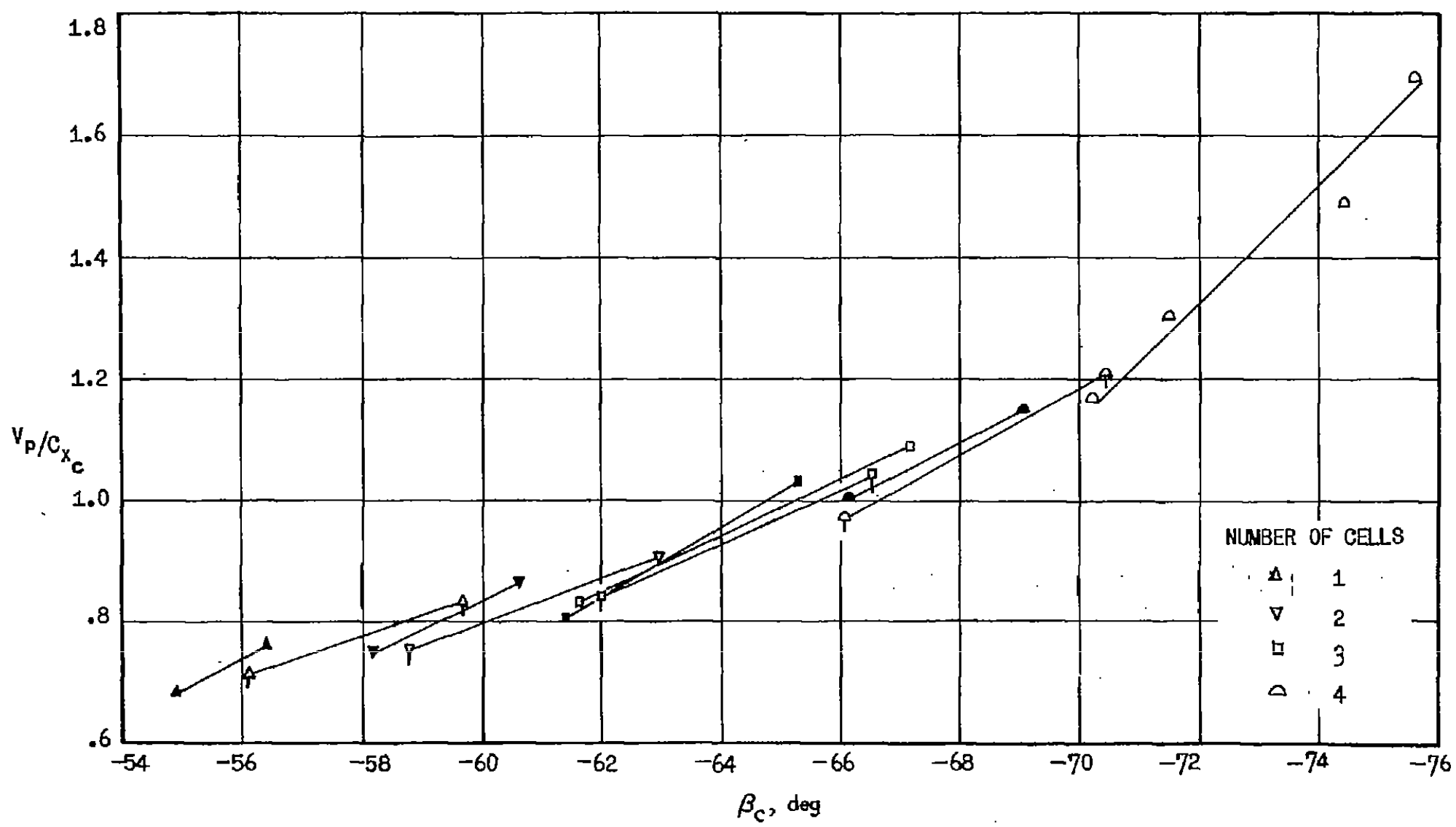

Flgure 52. - Plot of $\mathrm{V}_{\mathrm{p}} / \mathrm{C}_{\mathrm{x}_{\mathrm{C}}}$ versus $\beta_{\mathrm{C}}$ : Open symbols indicate date obtained at $500 \mathrm{rpm}$; solid symbols, 1,500 rpm; flagged symbols, 2,500 rpm. 


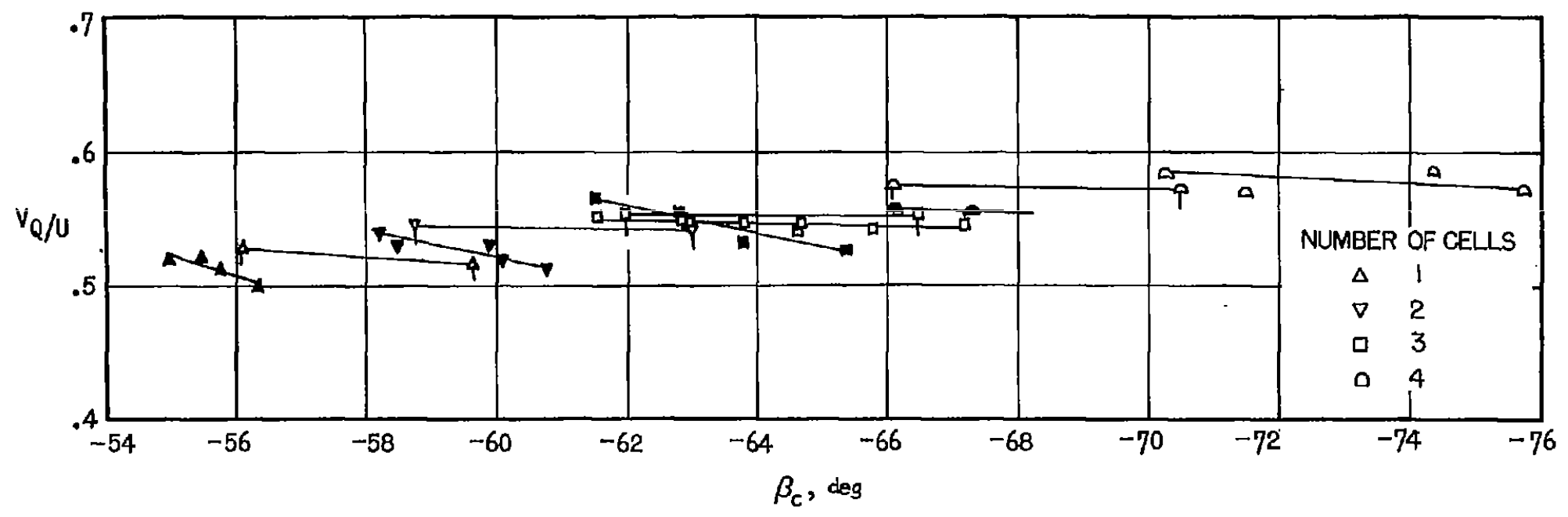

Figure 53.- Plot of $\mathrm{V}_{Q} j \mathrm{U}$ versus $\beta_{\mathrm{C}}$. Open symbols indicate data obtained at $500 \mathrm{rpm}$; solld symbols, 1,500 rpm; flagged symbols, 2,500 rpm. 


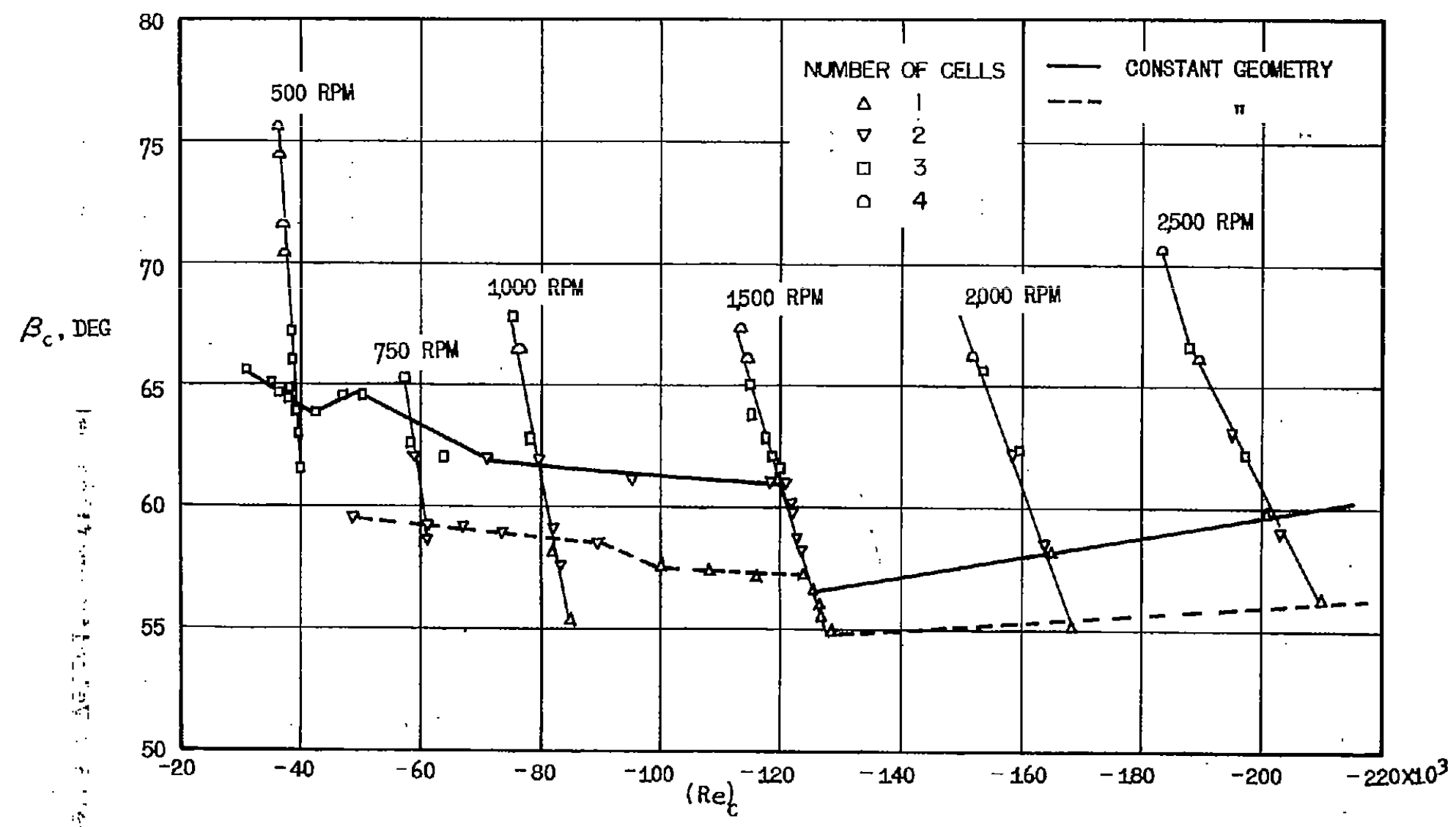

Figure 54.- Plot of $\beta_{C}$ versus Reynolds number. 


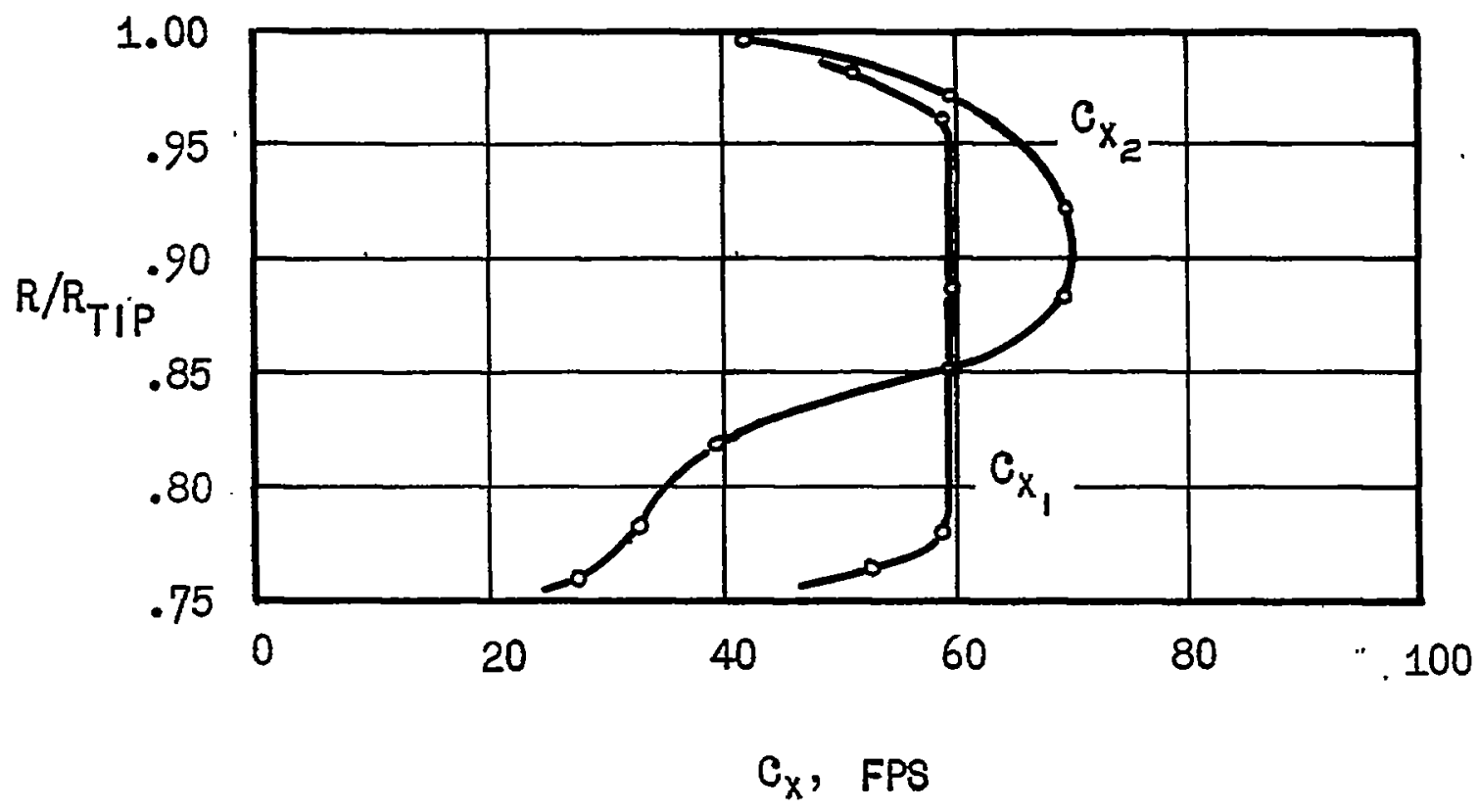

(a) Béfore stall propagation.

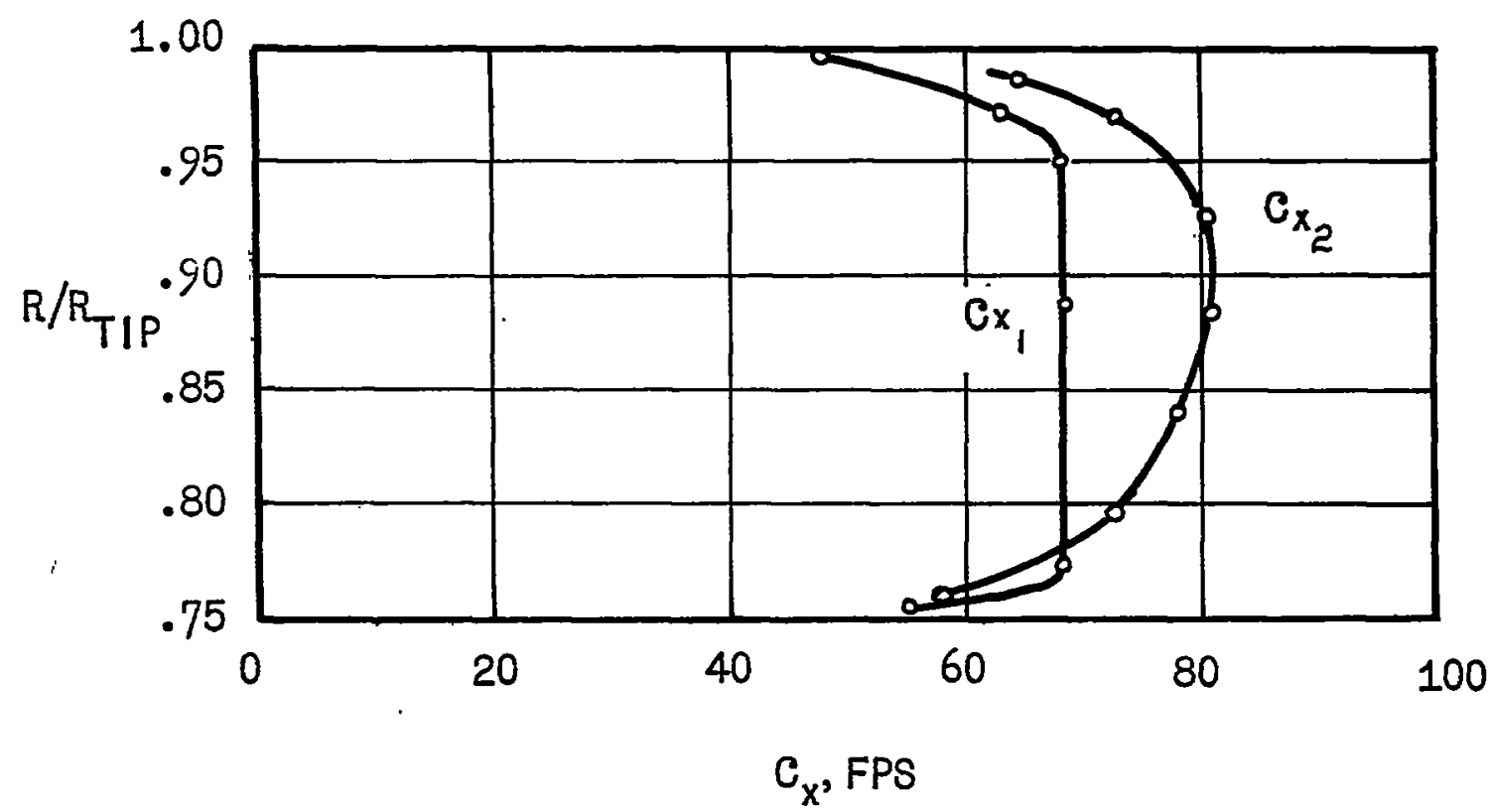

(b) During stall propagation.

Figure 55.- Velocity profiles before and after rotor. Configuration C. 


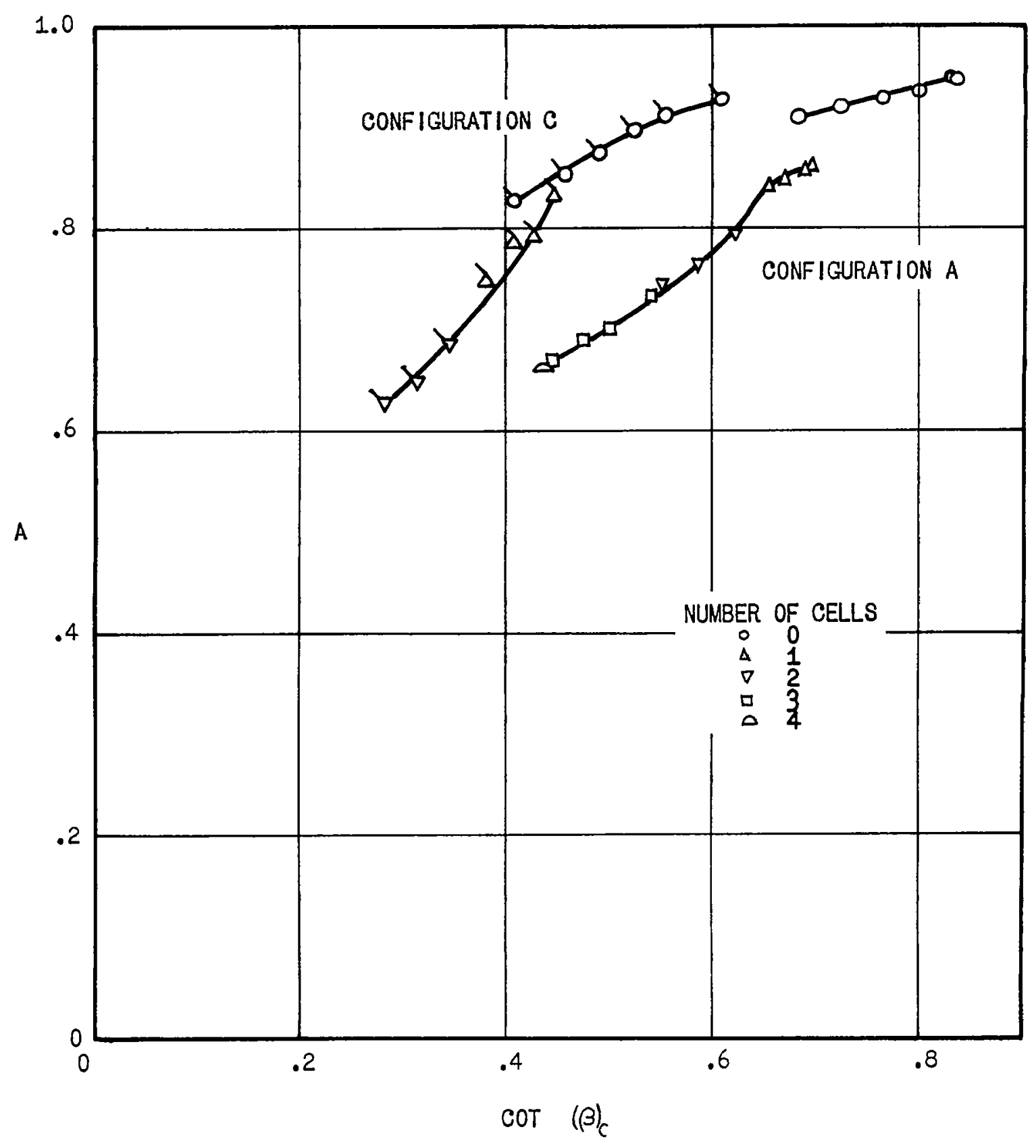

Figure 56.- Plot of discharge coefflclent A versus cot $(\beta)_{C}$. Flagged symbols indicate configuration $C$. 


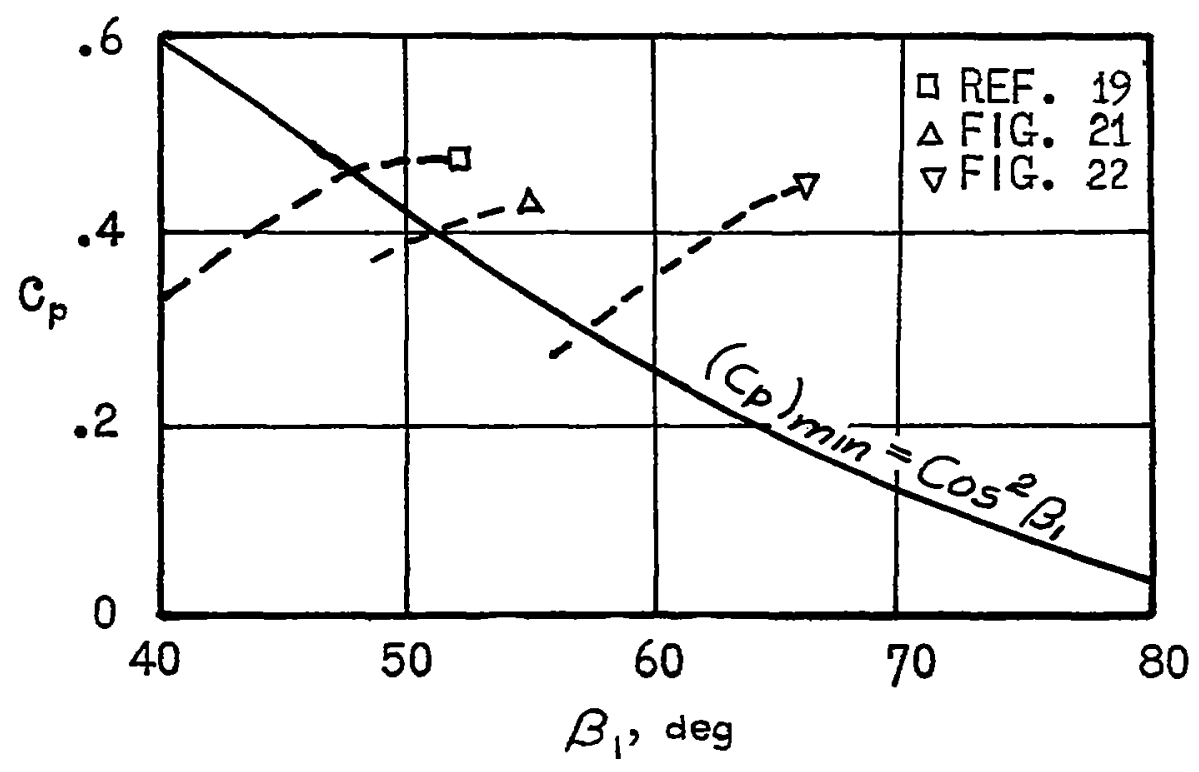

FIgure 57.- Minimum $C_{p}$ for stall propagation versus $\beta_{l}$. 


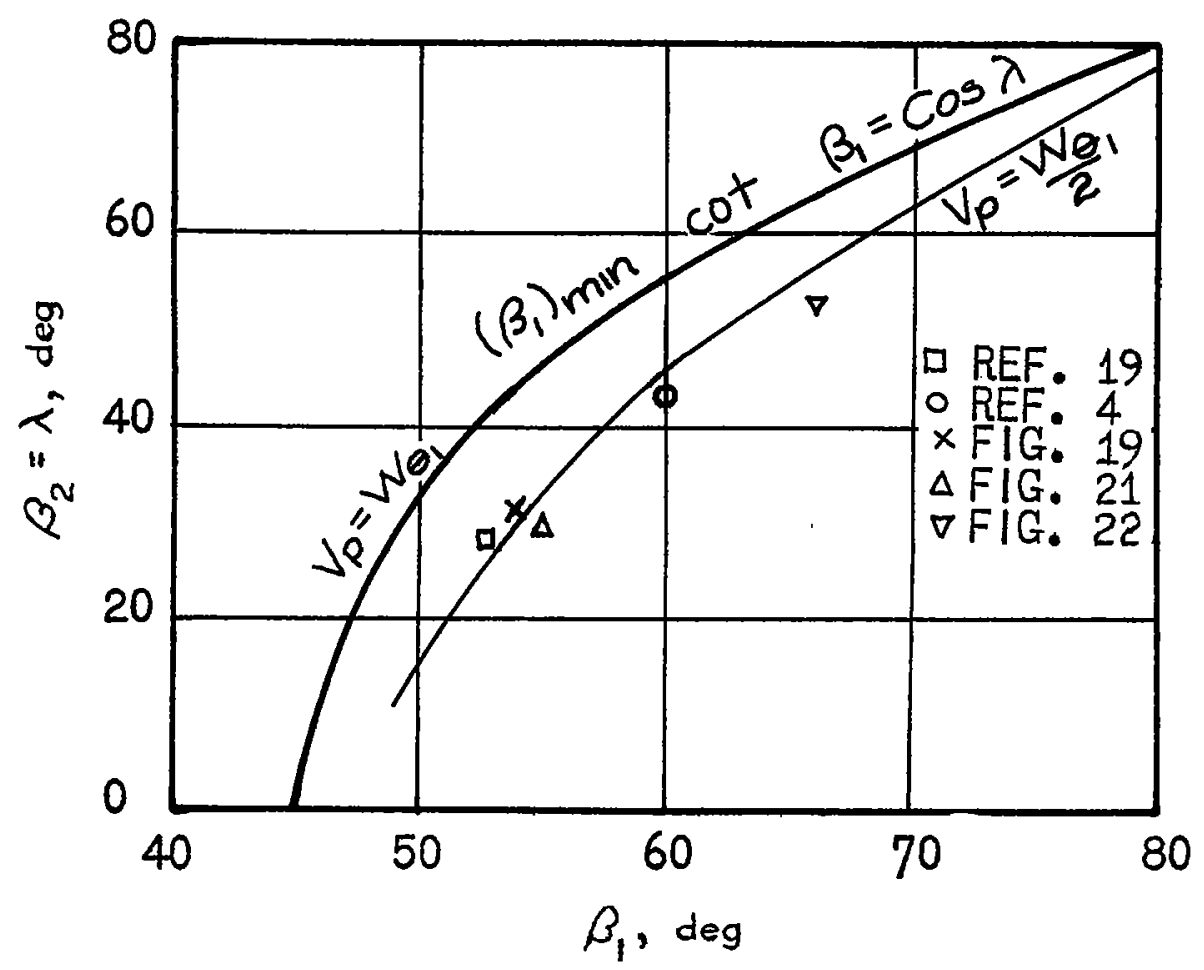

Figure 58.- Mintmum $\beta_{1}$ for stall propagation versus $\lambda$. 